\title{
Minimal dilatations of pseudo-Anosovs generated by the magic 3-manifold and their asymptotic behavior
}

\author{
EIKO KIN \\ SADAYOSHI KOJIMA \\ MitsuHiko TAKASAWA
}

This paper concerns the set $\widehat{\mathcal{M}}$ of pseudo-Anosovs which occur as monodromies of fibrations on manifolds obtained from the magic 3-manifold $N$ by Dehn filling three cusps with a mild restriction. Let $N(r)$ be the manifold obtained from $N$ by Dehn filling one cusp along the slope $r \in \mathbb{Q}$. We prove that for each $g$ (resp. $g \not \equiv 0(\bmod 6))$, the minimum among dilatations of elements (resp. elements with orientable invariant foliations) of $\widehat{\mathcal{M}}$ defined on a closed surface $\Sigma_{g}$ of genus $g$ is achieved by the monodromy of some $\Sigma_{g}$-bundle over the circle obtained from $N\left(\frac{3}{-2}\right)$ or $N\left(\frac{1}{-2}\right)$ by Dehn filling both cusps. These minimizers are the same ones identified by Hironaka, Aaber and Dunfield, Kin and Takasawa independently. In the case $g \equiv 6(\bmod 12)$ we find a new family of pseudo-Anosovs defined on $\Sigma_{g}$ with orientable invariant foliations obtained from $N(-6)$ or $N(4)$ by Dehn filling both cusps. We prove that if $\delta_{g}^{+}$is the minimal dilatation of pseudo-Anosovs with orientable invariant foliations defined on $\Sigma_{g}$, then

$$
\limsup _{g \equiv \underset{g \rightarrow \infty}{(\bmod 12)}} g \log \delta_{g}^{+} \leq 2 \log \delta\left(D_{5}\right) \approx 1.0870,
$$

where $\delta\left(D_{n}\right)$ is the minimal dilatation of pseudo-Anosovs on an $n$-punctured disk. We also study monodromies of fibrations on $N(1)$. We prove that if $\delta_{1, n}$ is the minimal dilatation of pseudo-Anosovs on a genus 1 surface with $n$ punctures, then

$$
\limsup _{n \rightarrow \infty} n \log \delta_{1, n} \leq 2 \log \delta\left(D_{4}\right) \approx 1.6628 .
$$

57M27, 37E30; 37B40

\section{Introduction}

\subsection{Minimal dilatations of pseudo-Anosovs}

Let $\operatorname{Mod}(\Sigma)$ be the mapping class group of a connected oriented surface $\Sigma$, and let $\phi \in \operatorname{Mod}(\Sigma)$ be a pseudo-Anosov class. Then $\phi \in \operatorname{Mod}(\Sigma)$ contains as a representative 
a pseudo-Anosov homeomorphism $\Phi: \Sigma \rightarrow \Sigma$ equipped with a constant $\lambda=\lambda(\Phi)>1$ called the dilatation of $\Phi$. The dilatation $\lambda(\phi)$ of $\phi$ is defined to be $\lambda(\Phi)$. The topological entropy $\operatorname{ent}(\Phi)$ of $\Phi$ is equal to $\log \lambda(\Phi)$, and $\operatorname{ent}(\Phi)$ attains the minimal entropy among all homeomorphisms which are isotopic to $\Phi$; see Fathi, Laudenbach and Poenaru [9, Exposé 10]. We denote this characteristic number by ent $(\phi)$, and call it the entropy of $\phi$. We call $\operatorname{Ent}(\phi)=|\chi(\Sigma)| \operatorname{ent}(\phi)$ the normalized entropy of $\phi$.

If we fix $\Sigma$,

$$
\{\operatorname{ent}(\phi) \mid \phi \in \operatorname{Mod}(\Sigma) \text { is pseudo-Anosov\} }
$$

is a closed discrete subset of $\mathbb{R}$; see Ivanov [16]. In particular there exists the minimum $\delta(\Sigma)>1$ among dilatations of pseudo-Anosov elements in $\operatorname{Mod}(\Sigma)$. The explicit values of $\delta(\Sigma)$ were computed in several cases where $|\chi(\Sigma)|$ is small; see Cho and Ham [5], Ham and Song [12], Song, Ko and Los [29] and Lanneau and Thiffeault [20]. For example, if $D_{n}$ is an $n$-punctured disk, then $\delta\left(D_{3}\right)=(3+\sqrt{5}) / 2 \approx 2.6180$, $\delta\left(D_{4}\right) \approx 2.2966$ is equal to the largest real root of $t^{4}-2 t^{3}-2 t+1$ and $\delta\left(D_{5}\right) \approx 1.7220$ is equal to the largest real root of $t^{5}-2 t^{3}-2 t^{2}+1$. However, it is widely open to determine $\delta(\Sigma)$ for most surfaces $\Sigma$.

Let $\Sigma_{g}$ be a closed surface of genus $g$, and let $\Sigma_{g, n}$ be a compact surface of genus $g$ with $n$ boundary components. We set $\delta_{g}=\delta\left(\Sigma_{g}\right)$ and $\delta_{g, n}=\delta\left(\Sigma_{g, n}\right)$. Penner proved that $\log \delta_{g} \asymp \frac{1}{g}$ [28]. It is an open problem to compute $\delta_{g}$ for $g>2$, but some partial results are known. Let $\delta_{g}^{+}$be the minimal dilatation of pseudo-Anosov homeomorphisms on $\Sigma_{g}$ with orientable invariant foliations. The explicit values $\delta_{g}^{+}$are known for all $2 \leq g \leq 8$ except for $g=6$; see Zhirov [35], Lanneau and Thiffeault [21], Aaber and Dunfield [1], Hironaka [14] and Kin and Takasawa [19].

We are motivated by the following question, posed by McMullen, which asks about the asymptotic behavior of the sequence $\left\{\delta_{g}\right\}_{g \geq 2}$.

Question 1.1 (McMullen [26]) Does $\lim _{g \rightarrow \infty} g \log \delta_{g}$ exist? What is its value?

It was proved by Minakawa [27] and independently by Hironaka and Kin [15] that $\log \delta_{g}^{+} \asymp \frac{1}{g}$, and by Tsai [32;33] that $\log \delta_{1, n} \asymp \frac{1}{n}$. Thus we can also ask the following.

Question 1.2 Does $\lim _{g \rightarrow \infty} g \log \delta_{g}^{+}$or $\lim _{n \rightarrow \infty} n \log \delta_{1, n}$ exist? What is its value?

Penner's lower bound on $\delta_{g, n}$ in [28] gives a uniform lower bound $\frac{\log 2}{12}<g \log \delta_{g} \leq$ $g \log \delta_{g}^{+}$and $\frac{\log 2}{4} \leq n \log \delta_{1, n}$.

The purpose of this paper is to provide not a complete but a considerably sharp answer to Questions 1.1 and 1.2. To explain what we prove and why we believe it is very close to the sharp answer more precisely, we would like to give a rather long introduction. 


\subsection{Thurston norm and fibered 3-manifolds}

Let $M$ be an oriented 3-manifold with boundary $\partial M$ (possibly $\partial M=\varnothing$ ). Thurston discovered a pseudonorm

$$
\|\cdot\|: H_{2}(M, \partial M ; \mathbb{R}) \rightarrow \mathbb{R} .
$$

When $M$ is a hyperbolic 3-manifold, $\|\cdot\|$ becomes a norm. Moreover when $M$ fibers over the circle, he described a relation between $\|\cdot\|$ and fibrations on $M$ as we recall below. (For more details, see Thurston [31].)

The Thurston norm $\|\cdot\|$ is defined for an integral class $a \in H_{2}(M, \partial M ; \mathbb{Z})$ by

$$
\|a\|=\min _{F}\{-\chi(F)\},
$$

where the minimum is taken over all oriented surfaces $F$ embedded in $M$, satisfying $a=[F]$, with no components of nonnegative Euler characteristic. The surface $F$ which realizes this minimum is called the minimal representative of $a$, denoted by $F_{a}$. The norm $\|\cdot\|$ defined on integral classes admits a unique continuous extension $\|\cdot\|: H_{2}(M, \partial M ; \mathbb{R}) \rightarrow \mathbb{R}$ which is linear on rays through the origin. The unit ball $U_{M}=\left\{a \in H_{2}(M, \partial M ; \mathbb{R}) \mid\|a\| \leq 1\right\}$ is a compact, convex polyhedron [31].

Suppose that $M$ is a surface bundle over the circle and let $F$ be its fiber. The fibration determines a cohomology class $a^{*} \in H^{1}(M ; \mathbb{Z}) \cong\left[M, S^{1}\right]$, and hence a homology class $a \in H_{2}(M, \partial M ; \mathbb{Z})$ by Poincaré duality. Thurston proved in [31] that there exists a top-dimensional face $\Omega$ on $\partial U_{M}$ such that $[F]$ is an integral class of $\operatorname{int}\left(C_{\Omega}\right)$, where $C_{\Omega}$ is the cone over $\Omega$ with the origin and $\operatorname{int}\left(C_{\Omega}\right)$ is its interior. Moreover he proved that the minimal representative $F_{a}$ for any integral class $a$ in $\operatorname{int}\left(C_{\Omega}\right)$ becomes a fiber of the fibration associated to $a$. Because of this result, $\Omega$ is called a fibered face of $M$, and an integral class $a \in \operatorname{int}\left(C_{\Omega}\right)$ is called a fibered class. This property shows that if a hyperbolic 3-manifold with the second Betti number more than 1 admits a fibration over the circle, then it admits an infinite family of fibrations over the circle. If $a \in \operatorname{int}\left(C_{\Omega}\right)$ is a primitive integral class, then the associated fibration on $M$ has a connected fiber represented by $F_{a}$. Since $M$ is hyperbolic, the mapping class $\phi_{a}=\left[\Phi_{a}\right]$ of the monodromy $\Phi_{a}: F_{a} \rightarrow F_{a}$ is pseudo-Anosov due to the hyperbolization theorem by Thurston [30]. In particular, a single fibered 3-manifold could offer infinitely many pseudo-Anosovs defined on surfaces with variable topology.

Let us fix a fibered face $\Omega$ of $M$. The set of integral classes (hence fibered classes) and rational classes of $\operatorname{int}\left(C_{\Omega}\right)$ are denoted by $\operatorname{int}\left(C_{\Omega}(\mathbb{Z})\right)$ and $\operatorname{int}\left(C_{\Omega}(\mathbb{Q})\right)$ respectively. Let $a \in \operatorname{int}\left(C_{\Omega}(\mathbb{Z})\right)$ be a primitive class. The dilatation $\lambda(a)$ and entropy $\operatorname{ent}(a)=\log \lambda(a)$ are defined as the dilatation and entropy of the pseudo-Anosov mapping class $\phi_{a}$ respectively. The entropy defined on primitive fibered classes is extended 
to rational classes as follows: for a rational number $r$ and a primitive fibered class $a$, the entropy ent $(r a)$ is defined by $\frac{1}{|r|} \operatorname{ent}(a)$. Fried proved that $1 /$ ent: $\operatorname{int}\left(C_{\Omega}(\mathbb{Q})\right) \rightarrow \mathbb{R}$ is concave [10], and in particular ent: $\operatorname{int}\left(C_{\Omega}(\mathbb{Q})\right) \rightarrow \mathbb{R}$ admits a unique continuous extension

$$
\text { ent: } \operatorname{int}\left(C_{\Omega}\right) \rightarrow \mathbb{R}
$$

Moreover, Fried proved that the restriction of ent to the open fibered face $\operatorname{int}(\Omega)$ is proper, namely, ent $(a)$ goes to $\infty$ as $a$ goes to a point on the boundary $\partial \Omega$. Note that $1 /$ ent: $\operatorname{int}\left(C_{\Omega}\right) \rightarrow \mathbb{R}$ is linear along each ray through the origin and cannot be strictly concave for this direction, but it is actually strictly concave for other directions. This refinement of concavity was proved originally by Matsumoto [24] and later by McMullen [26]. The strict concavity of $1 /$ ent on $\operatorname{int}(\Omega)$ implies that ent is strictly convex on $\operatorname{int}(\Omega)$ because ent is positive valued. Now, by the definition of ent, we see that

$$
\text { Ent }=\|\cdot\| \text { ent: } \operatorname{int}\left(C_{\Omega}\right) \rightarrow \mathbb{R}
$$

becomes constant on each ray in $\operatorname{int}\left(C_{\Omega}\right)$ through the origin. We call $\operatorname{Ent}(a)$ the normalized entropy of $a \in \operatorname{int}\left(C_{\Omega}\right)$. Since $\|\cdot\|$ is constant on a fibered face $\Omega$, the normalized entropy Ent is still strictly convex on $\operatorname{int}(\Omega)$. Thus because of the properness of ent by Fried, Ent admits a minimum at a unique ray through the origin. In other words, if we regard Ent as a function defined on $\operatorname{int}(\Omega)$, then it has a minimum at a unique point in $\operatorname{int}(\Omega)$. We denote this minimum by $\min \operatorname{Ent}(M, \Omega)$. We also denote by $\min \operatorname{Ent}(M)$, the minimum of $\{\min \operatorname{Ent}(M, \Omega) \mid \Omega$ is a fibered face of $M\}$.
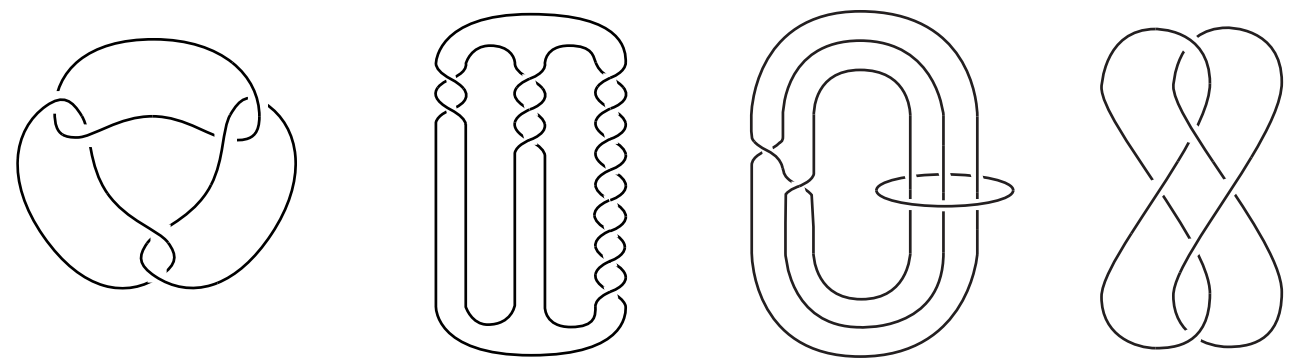

Figure 1: (From left to right) 3 chain $\operatorname{link} \mathcal{C}_{3},(-2,3,8)-$ pretzel link, link $6_{2}^{2}$, Whitehead link

\subsection{Finiteness of Farb, Leininger, Margalit and Agol}

We recall a result that connects pseudo-Anosovs having small dilatations with finitely many fibered 3-manifolds. For $P>1$, consider the following set of pseudo-Anosov 
homeomorphisms on any surface as follows:

$\Psi_{P}=\{\Phi \mid \Phi: \Sigma \rightarrow \Sigma$ pseudo-Anosov, $\chi(\Sigma)<0, \operatorname{Ent}(\Phi)=|\chi(\Sigma)| \operatorname{ent}(\Phi) \leq \log P\}$.

Farb, Leininger and Margalit called elements of $\Psi_{P}$ small dilatation pseudo-Anosov homeomorphisms. Let $\Sigma^{\circ} \subset \Sigma$ be the surface obtained by removing all the singularities of the stable foliation for $\Phi$, and $\left.\Phi\right|_{\Sigma^{\circ}}: \Sigma^{\circ} \rightarrow \Sigma^{\circ}$ denotes the restriction of $\Phi$ to $\Sigma^{\circ}$. Observe that $\lambda(\Phi)=\lambda\left(\left.\Phi\right|_{\Sigma^{\circ}}\right)$. Let us put

$$
\Psi_{P}^{\circ}=\left\{\left.\Phi\right|_{\Sigma^{\circ}} \mid \Phi \in \Psi_{P}\right\} .
$$

Penner's result implies that the set $\Psi_{P}$ is infinite if $P$ is large $\left(P \geq 11^{2}=121\right.$ for instance), and hence so is $\Psi_{P}^{\circ}$. However, Farb, Leininger and Margalit [8] and Agol [3] showed that if we let $\mathbb{T}\left(\Psi_{P}^{\circ}\right)$ be the set of mapping tori of elements of $\Psi_{P}^{\circ}$, then $\mathbb{T}\left(\Psi_{P}^{\circ}\right)$ becomes a finite set. In other words, for any $P>1$, there is a list of finitely many complete, noncompact hyperbolic 3-manifolds $M_{1}, M_{2}, \ldots, M_{r}$ fibering over the circle with the following property: for any $\Phi \in \Psi_{P}$, there exist $M_{i}$ in the list and a particular fibration on $M_{i}$ such that $\Phi$ occurs as the monodromy of the fibration on the manifold obtained from $M_{i}$ by Dehn filling along boundary slopes of the fiber in question.

Because of this, it makes sense to say that small dilatation pseudo-Anosovs are "generated" by a finite list of fibered 3-manifolds. This in particular implies that the following sets are finite because $\log \delta_{g} \asymp \frac{1}{g}$ and $\log \delta\left(D_{n}\right) \asymp \frac{1}{n}$ by Hironaka and Kin [15]:

$$
\begin{aligned}
& \mathcal{U}=\left\{\mathbb{T}\left(\left.\Phi\right|_{\Sigma^{\circ}}\right) \mid \Phi \text { is pseudo-Anosov on } \Sigma=\Sigma_{g} \text { such that } \lambda(\Phi)=\delta_{g}, g \geq 2\right\}, \\
& \mathcal{V}=\left\{\mathbb{T}\left(\left.\Phi\right|_{\Sigma^{\circ}}\right) \mid \Phi \text { is pseudo-Anosov on } \Sigma=D_{n} \text { such that } \lambda(\Phi)=\delta\left(D_{n}\right), n \geq 3\right\} .
\end{aligned}
$$

A natural question arises: how large are these sets? By Aaber and Dunfield [1], Hironaka [14], the authors [17; 18; 19] and Venzke [34], we predict that $\mathcal{U}$ and $\mathcal{V}$ are quite small.

A result by Kin and Takasawa [18] says that the magic manifold $N$, which is the exterior of the 3 chain link $\mathcal{C}_{3}$ (see Figure 1), is a member of $\mathcal{V}$. More concretely, in [18] it was shown that for each $3 \leq n \leq 8$ (resp. $n \geq 9$ ), there exists a pseudo-Anosov homeomorphism $\Phi_{n}: D_{n} \rightarrow D_{n}$ with the smallest dilatation (resp. the smallest known dilatation) which is generated by $N$. The set $\mathcal{V}$ might consist of a single element $N$ indeed. Let us turn to the set $\mathcal{U}$. Potential examples of members of $\mathcal{U}$ are of the form $N(r)$, which is the manifold obtained from the magic manifold $N$ by Dehn filling one cusp along the slope $r \in \mathbb{Q} \cup\left\{\frac{1}{0}\right\}$. It was proved in Aaber and Dunfield [1], Hironaka [14] and the first and third authors [19] that there exists a pseudo-Anosov homeomorphism on $\Sigma_{g}$ for $g \geq 3$ with small dilatation generated by $N\left(\frac{3}{-2}\right)$ or by 
$N\left(\frac{1}{-2}\right)$. The manifolds $N\left(\frac{3}{-2}\right)$ and $N\left(\frac{1}{-2}\right)$ are the Whitehead sister link (ie $(-2,3,8)-$ pretzel link) exterior and the $6_{2}^{2}$ link exterior respectively (see Figure 1). What Kin and Takasawa proved in [19, Theorem 1.5] is the following.

Theorem 1.3 Let $r \in\left\{\frac{3}{-2}, \frac{1}{-2}, 2\right\}$. For each $g \geq 3$, there exist $\Sigma_{g}$-bundles over the circle obtained from $N(r)$ by Dehn filling both cusps along boundary slopes of fibers of $N(r)$. Among them, there exist monodromies $\Phi_{g}(r): \Sigma_{g} \rightarrow \Sigma_{g}$ of the fibrations such that

$$
\lim _{g \rightarrow \infty} g \log \lambda\left(\Phi_{g}(r)\right)=\log \delta\left(D_{3}\right)=\log \left(\frac{3+\sqrt{5}}{2}\right) .
$$

As a corollary, we have the following estimate from above, which was proved by Hironaka first [14]:

$$
\limsup _{g \rightarrow \infty} g \log \delta_{g} \leq \log \left(\frac{3+\sqrt{5}}{2}\right) .
$$

Theorem 1.3 is also established by Hironaka for $r=\frac{1}{-2}$ in [14] and Aabar and Dunfield for $r=\frac{3}{-2}$ in [1] independently.

\subsection{Thurston norm and Teichmüller polynomial of the magic manifold}

In view of the results in previous two subsections, we will focus only on the magic manifold $N$ and present various computational results. To do this, we discuss some detailed description of $N$ in this subsection.

Let $K_{\alpha}, K_{\beta}$ and $K_{\gamma}$ be the components of the 3 chain link $\mathcal{C}_{3}$. The orientation of each component of $\mathcal{C}_{3}$ is given in Figure 2(right). They bound the oriented disks $F_{\alpha}, F_{\beta}$ and $F_{\gamma}$ with 2 holes. Let us set $\alpha=\left[F_{\alpha}\right], \beta=\left[F_{\beta}\right], \gamma=\left[F_{\gamma}\right] \in H_{2}(N, \partial N ; \mathbb{Z})$. In [31], Thurston computed the unit ball $U_{N}$ which is the parallelepiped with vertices $\pm \alpha, \pm \beta, \pm \gamma$ and $\pm(\alpha+\beta+\gamma)$; see Figure 2 (left). The set $\{\alpha, \beta, \gamma\}$ is a basis of $H_{2}(N, \partial N ; \mathbb{Z})$, and the class $x \alpha+y \beta+z \gamma \in H_{2}(N, \partial N)$ is denoted by $(x, y, z)$. Every top-dimensional face on $\partial U_{N}$ is a fibered face because of the symmetries of $H_{2}(N, \partial N)$; see Section 2.1. McMullen developed a general theory of the Teichmüller polynomial $P_{\Omega}$ for a fibered face $\Omega$ of fibered hyperbolic 3manifolds, from which one can compute the dilatation $\lambda(a)$ of each $a \in \operatorname{int}\left(C_{\Omega}\right)$; see [26]. Let us pick the fibered face $\Delta$ on $\partial U_{N}$ as in Figure 2(left) with vertices $(1,0,0),(1,1,1),(0,1,0)$ and $(0,0,-1)$. The Teichmüller polynomial $P_{\Delta}$ tells us that the dilatation $\lambda(a)=\lambda_{(x, y, z)}$ of a primitive fibered class $a=(x, y, z) \in \operatorname{int}\left(C_{\Delta}\right)$ is the largest real root of

$$
f_{(x, y, z)}(t)=t^{x+y-z}-t^{x}-t^{y}-t^{x-z}-t^{y-z}+1 ;
$$


see [18, Theorem 3.1]. Thus, we have a reasonable source to compute dilatations systematically.
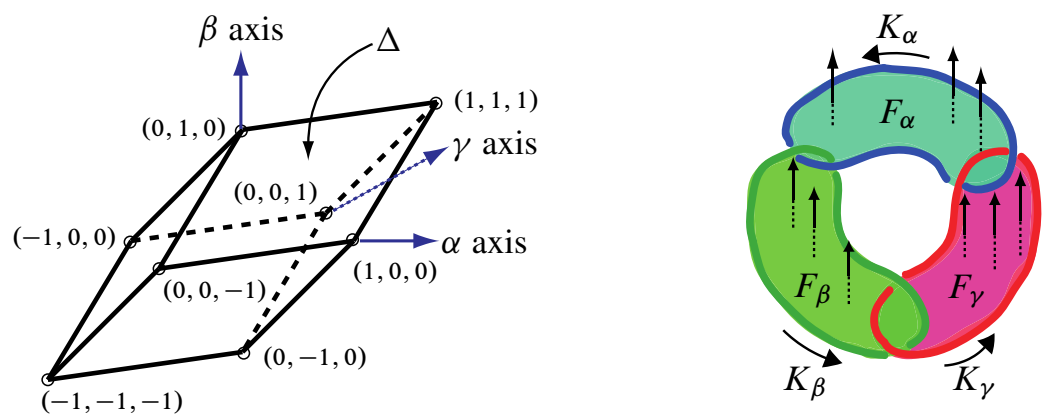

Figure 2: (left) Thurston norm ball for N, (right) $F_{\alpha}, F_{\beta}, F_{\gamma}$ [arrows indicate the normal direction of oriented surfaces]

To relate these data to ones on closed manifolds obtained from $N$ by Dehn filling, we prepare a few homological properties of $N$. Denote by $T_{\alpha}$ the torus which is the boundary of a regular neighborhood of $K_{\alpha}$. We define the tori $T_{\beta}$ and $T_{\gamma}$ in the same manner. For a primitive integral class $a=(x, y, z) \in H_{2}(N, \partial N)$, let us set $\partial_{\alpha} F_{a}=\partial F_{a} \cap T_{\alpha}$ which consists of the parallel simple closed curves on $T_{\alpha}$. We define $\partial_{\beta} F_{a}$ and $\partial_{\gamma} F_{a}$ in the same manner. We see that the slope of $\partial_{\alpha} F_{a}$ (resp. $\left.\partial_{\beta} F_{a}, \partial_{\gamma} F_{a}\right)$ is given by $b_{\alpha}(a)=\frac{y+z}{-x}\left(\operatorname{resp} . b_{\beta}(a)=\frac{z+x}{-y}, b_{\gamma}(a)=\frac{x+y}{-z}\right)$. We call each of $b_{\alpha}(a), b_{\beta}(a), b_{\gamma}(a)$ the boundary slope of $a$.

For more detailed computation, we specify the cusp to be Dehn filled. Let $N(r)$ be the manifold obtained from $N$ by Dehn filling the cusp specified, say, by $T_{\beta}$ along the slope $r \in \mathbb{Q}$. Then, there exists a natural injection $\iota_{\beta}: H_{2}(N(r), \partial N(r)) \rightarrow H_{2}(N, \partial N)$ whose image equals $S_{\beta}(r)$, where

$$
S_{\beta}(r)=\left\{(x, y, z) \in H_{2}(N, \partial N) \mid-r y=z+x\right\} ;
$$

see Proposition 2.11. This implies that every slope $r \in \mathbb{Q}$ can be realized by a boundary slope of some $a \in H_{2}(N, \partial N)$. It is known by Martelli and Petronio [23] that $N(r)$ is hyperbolic if and only if $r \in \mathcal{H} y p=\mathbb{Q} \backslash\{-3,-2,-1,0\}$. Choose such $r \in \mathcal{H} y p$, and assume that $a \in S_{\beta}(r)=\operatorname{Im} \iota_{\beta}$ is a fibered class in $H_{2}(N, \partial N)$. Then, $\bar{a}=\iota_{\beta}^{-1}(a) \in H_{2}(N(r), \partial N(r))$ is also a fibered class of $N(r)$. This description enables us to compute the Thurston norm of $N(r)$, especially the unit ball and fibered faces, and hence to handle closed surface bundles obtained from $N$ by Dehn filling all cusps systematically.

What we further need for our purpose is to know a systematic method to compute entropies of monodromies on such bundles. 


\subsection{Main results}

This paper concerns the small dilatation pseudo-Anosovs generated by the magic manifold $N$ with a very mild restriction which we describe below. Let $\Phi: F \rightarrow F$ be the monodromy of a fibration on $N$, and let $\phi$ be the mapping class of $\Phi$. Then the fibration extends naturally to a fibration on the closed manifold obtained from $N$ by Dehn filling three cusps along boundary slopes of $F$. Also, $\Phi$ extends to the monodromy $\widehat{\Phi}: \hat{F} \rightarrow \widehat{F}$ of the extended fibration, where the extended fiber $\widehat{F}$ is obtained from $F$ by filling holes. Suppose that the stable foliation $\mathcal{F}$ of $\Phi$ has the property such that each boundary component of $F$ has no 1 prong. Then $\mathcal{F}$ extends canonically to the stable foliation $\widehat{\mathcal{F}}$ of $\widehat{\Phi}$, and $\widehat{\phi}=[\widehat{\Phi}]$ becomes pseudo-Anosov (including Anosov) with the same dilatation as that of $\phi$. We consider the set $\mathcal{M}$ of (pseudo-Anosov) mapping classes coming from fibrations of $N$ with this condition, ie, if we let $\mathcal{F}$ be the stable foliation associated to the fibration on $N$, then $\mathcal{F}$ has the property such that

any boundary component of $F$ has no 1 prong.

We will see that this restriction is fairly mild (Lemmas 2.23 and 5.1). Let $\widehat{\mathcal{M}}$ be the set of extensions $\hat{\phi}$ of $\phi \in \mathcal{M}$ defined on the closed surfaces. For example, the pseudo-Anosov $\Phi_{g}(r): \Sigma_{g} \rightarrow \Sigma_{g}$ in Theorem 1.3 enjoys $\left[\Phi_{g}(r)\right] \in \widehat{\mathcal{M}}$ for large $g$; see [19, Lemma 4.8].

Let $\widehat{\delta}_{g}$ be the minimum among dilatations of elements in $\widehat{\mathcal{M}} \cap \operatorname{Mod}\left(\Sigma_{g}\right)$. Clearly we have that $\delta_{g} \leq \hat{\delta}_{g}$. Equality is achieved when $g=2$ [14]. We prove the limit of $g \log \widehat{\delta}_{g}$ exists and it equals the number which we encountered in Theorem 1.3.

Theorem 1.4 (1) We have $\lim _{g \rightarrow \infty} g \log \widehat{\delta}_{g}=\log \left(\frac{3+\sqrt{5}}{2}\right)$.

(2) For large $g, \widehat{\delta}_{g}$ is achieved by the monodromy of some $\Sigma_{g}$-bundle over the circle obtained from either $N\left(\frac{3}{-2}\right)$ or $N\left(\frac{1}{-2}\right)$ by Dehn filling both cusps.

More precisely, the following holds (see also Remark 3.18). For large $g$ such that $g \equiv 0,1,5,6,7,9(\bmod 10)($ resp. large $g$ such that $g \equiv 3,8(\bmod 10)), \widehat{\delta}_{g}$ is achieved by the monodromy of some $\Sigma_{g}$-bundle over the circle obtained from $N\left(\frac{3}{-2}\right)$ (resp. $\left.N\left(\frac{1}{-2}\right)\right)$ by Dehn filling both cusps.

We know from [19, Proposition 4.37] that for $g=8,13, \hat{\delta}_{g}$ cannot be achieved by the monodromy of any $\Sigma_{g}$-bundle over the circle obtained from either $N\left(\frac{3}{-2}\right)$ or $N\left(\frac{1}{-2}\right)$ by Dehn filling. In fact, the manifold $N\left(\frac{4}{-3}, \frac{25}{-17},-5\right)$ (resp. $\left.N\left(\frac{29}{-27}, \frac{5}{-3},-6\right)\right)$ is a $\Sigma_{8}$-bundle (resp. a $\Sigma_{13}$-bundle) over the circle, where $N\left(r_{1}, r_{2}, r_{3}\right)$ is the closed 
manifold obtained from $N$ by Dehn filling all cusps along the slopes $r_{1}, r_{2}$ and $r_{3}$. Its dilatation is smaller than that of any $\Sigma_{8}$-bundle (resp. $\Sigma_{13}$-bundle) over the circle obtained from either $N\left(\frac{3}{-2}\right)$ or $N\left(\frac{1}{-2}\right)$ by Dehn filling. Theorem 1.4 says if $g$ is large, then among the elements of $\widehat{\mathcal{M}}$, the pseudo-Anosovs defined on $\Sigma_{g}$ with the smallest dilatation are the same examples identified by Hironaka [14], Aabor and Dunfield [1] and Kin and Takasawa [19].

However, we can find new examples in $\widehat{\mathcal{M}}$ defined on $\Sigma_{g}$ with orientable invariant foliations when $g \equiv 0(\bmod 6)$. Let $\widehat{\mathcal{M}}^{+}$be the set of pseudo-Anosov elements of $\widehat{\mathcal{M}}$ with orientable invariant foliations. Let $\hat{\delta}_{g}^{+}$be the minimum among dilatations of elements in $\widehat{\mathcal{M}}^{+} \cap \operatorname{Mod}\left(\Sigma_{g}\right)$. Since $\widehat{\mathcal{M}}^{+} \cap^{\circ} \operatorname{Mod}\left(\Sigma_{g}\right) \neq \varnothing$ for $g \geq 2$ (Lemma 3.19), then $\widehat{\delta}_{g}^{+}$is well-defined. Clearly $\delta_{g} \leq \delta_{g}^{+} \leq \hat{\delta}_{g}^{+}$.

The following describes the asymptotic behavior of $\hat{\delta}_{g}^{+}$'s in the case $g \not \equiv 0(\bmod 6)$.

Theorem 1.5 (1) We have

$$
\lim _{\substack{g \neq 0(\bmod 6) \\ g \rightarrow \infty}} g \log \widehat{\delta}_{g}^{+}=\log \left(\frac{3+\sqrt{5}}{2}\right) .
$$

(2) For large $g$ such that $g \equiv 2,4(\bmod 6)$ or $g \equiv 3(\bmod 10), \hat{\delta}_{g}^{+}$is achieved by the monodromy of some $\Sigma_{g}$-bundle over the circle obtained from $N\left(\frac{1}{-2}\right)$ by Dehn filling both cusps.

(3) For large $g$ such that $g \equiv 1,5,7,9(\bmod 10), \hat{\delta}_{g}^{+}$is achieved by the monodromy of some $\Sigma_{g}$-bundle over the circle obtained from $N\left(\frac{3}{-2}\right)$ by Dehn filling both cusps.

Theorem 1.5(1) leads to the following estimate, which was proved by Hironaka first [14].

$$
\limsup _{\substack{g \neq 0(\bmod 6) \\ g \rightarrow \infty}} g \log \delta_{g}^{+} \leq \log \left(\frac{3+\sqrt{5}}{2}\right) .
$$

If $g \not \equiv 0(\bmod 6)$ is large, then elements of $\widehat{\mathcal{M}}^{+}$defined on $\Sigma_{g}$ with the smallest dilatation are the same examples discovered in Hironaka [14], Aaber and Dunfield [1] and the first and third authors [19].

In the case $g \equiv 0(\bmod 6)$, there exist no examples of elements in $\widehat{\mathcal{M}}^{+}$defined on $\Sigma_{g}$ which occur as monodromies of fibrations on manifolds obtained from $N\left(\frac{1}{-2}\right)$ or $N\left(\frac{3}{-2}\right)$ by Dehn filling both cusps; see Hironaka [14], Aaber and Dunfield [1] and the first and third authors [19]. To the best of our knowledge, the smallest known upper bound on $\delta_{g}^{+}$for $g \equiv 0(\bmod 6)$ is

$$
\delta_{g}^{+} \leq \lambda_{(g, g,-1)}
$$


where $\lambda_{(g, g,-1)}$ is the largest root of $f_{(g, g,-1)}(t)=t^{2 g+1}-2 t^{g+1}-2 t^{g}+1[27 ; 15]$. By using the bound (1-4), Minakawa and independently Hironaka and Kin proved that

$$
g \log \delta_{g}^{+} \leq \log (2+\sqrt{3}) \approx 1.3169
$$

Note that the set $\widehat{\mathcal{M}}^{+}$could be a source to provide a sharper upper bound on $\delta_{g}^{+}$than the bound $(1-4)$ in the case $g \equiv 0(\bmod 6)$. In fact, we will find elements of $\widehat{\mathcal{M}}^{+}$ defined on $\Sigma_{g}$ for $g \equiv 6(\bmod 12)$ whose normalized entropies go to $4 \log \delta\left(D_{5}\right)$ as $g$ goes to $\infty$; see Lemmas 3.22 and 3.23. These examples occur as monodromies of fibrations on manifolds obtained from $N(4)$ or $N(-6)$ by Dehn filling both cusps. As a corollary, we have the following.

\section{Theorem 1.6 We have}

$$
\limsup _{g \equiv \underset{g \rightarrow \infty}{(\bmod 12)}} g \log \delta_{g}^{+} \leq 2 \log \delta\left(D_{5}\right) \approx 1.0870 .
$$

By using our examples, we give the following upper bound on $\delta_{g}^{+}$for $g \equiv 6(\bmod 12)$ which is sharper than the previous one (1-4) (see also Table 1).

Theorem 1.7 (Upper bound on $\delta_{g}^{+}$for $g \equiv 6(\bmod 12)$ )

(1) We have $\delta_{g}^{+} \leq \lambda_{((3 g / 2)+1,(3 g / 2)-1,(g / 2))}$ if $g \equiv 6,30,42,54,78(\bmod 84)$.

(2) We have $\delta_{g}^{+} \leq \lambda_{(g+2, g-2,-(g / 2))}$ if $g \equiv 18,66(\bmod 84)$.

In the case $g \equiv 0(\bmod 12)$, we improve the bound (1-4) for many $g$; see Table 1.

Section 3.5 concerns the monodromies of fibrations on the Whitehead link exterior $N(1)$. The manifold $N(1)$ is very special among other $N(r)$ 's. It is the only manifold among the $N(r)$ 's which admits fibers with arbitrarily many boundary components (Lemma 5.2). Moreover the invariant foliation of the monodromy of each fibration on $N(1)$ has the property such that each boundary component of the fiber has a 1 prong. (Remark 3.29). We shall show in Section 3.5 that there exists the monodromy $\Phi_{n}: \Sigma_{1, n} \rightarrow \Sigma_{1, n}$ of a particular fibration on $N(1)$ whose normalized entropy tends to $2 \log \delta\left(D_{4}\right)$ as $n$ tends to $\infty$ (Proposition 3.30 and Lemma 3.31). Thus we have the following.

Theorem $1.8 \limsup _{n \rightarrow \infty} n \log \delta_{1, n} \leq 2 \log \delta\left(D_{4}\right)$.

This implies the upper bound $\lim \sup _{n \rightarrow \infty} n \log \delta_{1, n} \leq 2 \log 9$ by Tsai; see [33, Section 3.2.1 and Theorem 3.2.2]. 


\begin{tabular}{|c|c|c|}
\hline$g$ & upper bound on $\delta_{g}^{+}$ & upper bound on $\delta_{g}^{+}$in $[27 ; 15]$ \\
\hline 6 & $\lambda_{(10,8,3)} \approx 1.20189$ & $\lambda_{(6,6,-1)} \approx 1.22571$ \\
\hline 12 & $\lambda_{(12,20,3)} \approx 1.10240$ & $\lambda_{(12,12,-1)} \approx 1.11124$ \\
\hline 18 & $\lambda_{(20,16,-9)} \approx 1.06276$ & $\lambda_{(18,18,-1)} \approx 1.07382$ \\
\hline 24 & $\lambda_{(32,28,3)} \approx 1.04757$ & $\lambda_{(24,24,-1)} \approx 1.05524$ \\
\hline 30 & $\lambda_{(46,44,15)} \approx 1.03692$ & $\lambda_{(30,30,-1)} \approx 1.04413$ \\
\hline 36 & $\lambda_{(50,52,15)} \approx 1.03148$ & $\lambda_{(36,36,-1)} \approx 1.03674$ \\
\hline 42 & $\lambda_{(64,62,21)} \approx 1.02622$ & $\lambda_{(42,42,-1)} \approx 1.03147$ \\
\hline 48 & $\lambda_{(66,68,19)} \approx 1.02367$ & $\lambda_{(48,48,-1)} \approx 1.02752$ \\
\hline 54 & $\lambda_{(82,80,27)} \approx 1.02033$ & $\lambda_{(54,54,-1)} \approx 1.02446$ \\
\hline 60 & $\lambda_{(80,76,15)} \approx 1.01903$ & $\lambda_{(60,60,-1)} \approx 1.02200$ \\
\hline 66 & $\lambda_{(68,64,-33)} \approx 1.01661$ & $\lambda_{(66,66,-1)} \approx 1.02000$ \\
\hline 72 & $\lambda_{(96,92,19)} \approx 1.01586$ & $\lambda_{(72,72,-1)} \approx 1.01833$ \\
\hline 78 & $\lambda_{(118,116,39)} \approx 1.01403$ & $\lambda_{(78,78,-1)} \approx 1.01691$ \\
\hline 84 & $\lambda_{(114,116,31)} \approx 1.01357$ & $\lambda_{(84,84,-1)} \approx 1.01570$ \\
\hline 90 & $\lambda_{(136,134,45)} \approx 1.01215$ & $\lambda_{(90,90,-1)} \approx 1.01465$ \\
\hline 96 & $\lambda_{(132,140,43)} \approx 1.01190$ & $\lambda_{(96,96,-1)} \approx 1.01374$ \\
\hline 102 & $\lambda_{(104,100,-51)} \approx 1.01071$ & $\lambda_{(102,102,-1)} \approx 1.01293$ \\
\hline 108 & $\lambda_{(146,148,39)} \approx 1.01057$ & $\lambda_{(108,108,-1)} \approx 1.01221$ \\
\hline 114 & $\lambda_{(172,170,57)} \approx 1.00958$ & $\lambda_{(114,114,-1)} \approx 1.01156$ \\
\hline 120 & $\lambda_{(164,172,51)} \approx 1.00952$ & $\lambda_{(120,120,-1)} \approx 1.01098$ \\
\hline 126 & $\lambda_{(190,188,63)} \approx 1.00841$ & $\lambda_{(126,126,-1)} \approx 1.01046$ \\
\hline 132 & $\lambda_{(174,164,31)} \approx 1.00869$ & $\lambda_{(132,132,-1)} \approx 1.00998$ \\
\hline 138 & $\lambda_{(208,206,69)} \approx 1.00790$ & $\lambda_{(138,138,-1)} \approx 1.00955$ \\
\hline 144 & $\lambda_{(194,196,51)} \approx 1.00793$ & $\lambda_{(144,144,-1)} \approx 1.00915$ \\
\hline 150 & $\lambda_{(152,148,-75)} \approx 1.00727$ & $\lambda_{(150,150,-1)} \approx 1.00878$ \\
\hline 156 & $\lambda_{(210,212,55)} \approx 1.00732$ & $\lambda_{(156,1566,-1)} \approx 1.00845$ \\
\hline 162 & $\lambda_{(244,, 242,81)} \approx 1.00673$ & $\lambda_{(162,162,-1)} \approx 1.00813$ \\
\hline 168 & $\lambda_{(228,236,67)} \approx 1.00680$ & $\lambda_{(168,168,-1)} \approx 1.00784$ \\
\hline 174 & $\lambda_{(262,260,87)} \approx 1.00626$ & $\lambda_{(174,174,-1)} \approx 1.00757$ \\
\hline 180 & $\lambda_{(240,236,55)} \approx 1.00635$ & $\lambda_{(180,180,-1)} \approx 1.00732$ \\
\hline 186 & $\lambda_{(188,184,-93)} \approx 1.00586$ & $\lambda_{(186,186,-1)} \approx 1.00708$ \\
\hline 192 & $\lambda_{(258,260,67)} \approx 1.00595$ & $\lambda_{(192,192,-1)} \approx 1.00686$ \\
\hline 198 & $\lambda_{(298,296,99)} \approx 1.00550$ & $\lambda_{(198,198,-1)} \approx 1.00665$ \\
\hline 204 & $\lambda_{(276,284,79)} \approx 1.00560$ & $\lambda_{(204,204,-1)} \approx 1.00646$ \\
\hline 210 & $\lambda_{(316,314,105)} \approx 1.00519$ & $\lambda_{(210,210,-1)} \approx 1.00627$ \\
\hline 216 & $\lambda_{(290,292,75)} \approx 1.00529$ & $\lambda_{(216,216,-1)} \approx 1.00610$ \\
\hline
\end{tabular}

Table 1: Upper bounds on $\delta_{g}^{+}$such that $g \equiv 0(\bmod 6)$ [the bounds on the left if $g \equiv 6(\bmod 12)$ come from Theorem 1.7 ; if $g \equiv 0(\bmod 12)$ and $g>12$, the bounds on the left are given by elements of $\widehat{\mathcal{M}}^{+}$which occur as monodromies of fibrations on manifolds obtained from $N\left(\frac{5}{-4}\right)$ by Dehn filling both cusps] 


\subsection{Thurston norm equivalence, entropy equivalence on manifolds $N(r)$}

In the course of analyzing the magic manifold, we discovered many "twins" among the $N(r)$ 's. The particular ones are $N\left(\frac{3}{-2}\right)$ and $N\left(\frac{1}{-2}\right)$ which will be critical in the proof of Theorem 1.4. They are different manifolds but have common properties from entropy computational viewpoints. To formulate ideas, we say that 3-manifolds $M$ and $M^{\prime}$ are Thurston norm equivalent, denoted by

$$
M \underset{\mathrm{T}}{\sim} M^{\prime},
$$

if there is an isomorphism $f: H_{2}(M, \partial M ; \mathbb{Z}) \rightarrow H_{2}\left(M^{\prime}, \partial M^{\prime} ; \mathbb{Z}\right)$ which preserves the Thurston norm, ie, $\|a\|=\|f(a)\|$ for any $a \in H_{2}(M, \partial M ; \mathbb{Z})$. We call such $f$ the Thurston norm preserving isomorphism. For example

$$
N(r) \underset{\mathrm{T}}{\sim} N(-2-r)
$$

when $r,-2-r \in \mathcal{H} y p$ (Proposition 2.20). We introduce two more equivalence relations, both called the entropy equivalence, of which the precise definitions are given in Section 2.5.1. The first one is defined on the pairs $(M, \Omega)$, where $M$ is a fibered 3 -manifold and $\Omega$ is its fibered face. Namely, $(M, \Omega)$ and $\left(M^{\prime}, \Omega^{\prime}\right)$ are entropy equivalent, denoted by

$$
(M, \Omega) \underset{\mathrm{ent}}{\sim}\left(M^{\prime}, \Omega^{\prime}\right),
$$

if we have that there is a Thurston norm preserving isomorphism $f: H_{2}(M, \partial M ; \mathbb{Z}) \rightarrow$ $H_{2}\left(M^{\prime}, \partial M^{\prime} ; \mathbb{Z}\right)$ such that $f$ maps $\operatorname{int}\left(C_{\Omega}(\mathbb{Z})\right)$ to $\operatorname{int}\left(C_{\Omega^{\prime}}(\mathbb{Z})\right)$ preserving the entropy function. In particular

$$
(M, \Omega) \underset{\text { ent }}{\sim}\left(M^{\prime}, \Omega^{\prime}\right)
$$

implies that $\min \operatorname{Ent}(M, \Omega)=\min \operatorname{Ent}\left(M^{\prime}, \Omega^{\prime}\right)$. The second equivalence relation is defined on the fibered 3-manifolds. Fibered 3-manifolds $M$ and $M^{\prime}$ are entropy equivalent, denoted by

$$
M \underset{\text { ent }}{\sim} M^{\prime}
$$

if we have that there is a Thurston norm preserving isomorphism $f: H_{2}(M, \partial M ; \mathbb{Z}) \rightarrow$ $H_{2}\left(M^{\prime}, \partial M^{\prime} ; \mathbb{Z}\right)$ such that $f$ preserves both fibered classes and the entropy functions. If $M \underset{\text { ent }}{\sim} M^{\prime}$, then $\min \operatorname{Ent}(M)=\min \operatorname{Ent}\left(M^{\prime}\right)$. We shall prove in Theorem 2.26 that

$$
\left(N(2), \Omega_{S}\right) \underset{\text { ent }}{\sim}\left(N\left(\frac{3}{-2}\right), \Omega_{A}\right) \underset{\text { ent }}{\sim}\left(N\left(\frac{1}{-2}\right), \Omega_{A}\right)
$$


For the definition of fibered faces $\Omega_{S}$ and $\Omega_{A}$; see Section 2.5.2. We also prove that

$$
N(r) \underset{\text { ent }}{\sim} N(-2-r)
$$

for 'almost all' $r \in \mathcal{H} y p$; see Theorem 2.26. This is derived from the symmetry of the Thurston norm ball and the symmetry of the entropy function of $N$. In particular

$$
N\left(\frac{3}{-2}\right) \underset{\mathrm{ent}}{\sim} N\left(\frac{1}{-2}\right) \text {. }
$$

Recall that the quantity min Ent is defined to be the minimum of the normalized entropies of the classes in $\bigcup_{\Omega} \operatorname{int}\left(C_{\Omega}\right)$, where $\Omega$ is taken over all fibered faces of $M$. The number $\log ((3+\sqrt{5}) / 2)$ in Theorem 1.3 appears in the equalities $\min \operatorname{Ent}\left(N\left(\frac{3}{-2}\right)\right)=\min \operatorname{Ent}\left(N\left(\frac{1}{-2}\right)\right)=\min \operatorname{Ent}\left(N(2), \Omega_{S}\right)=2 \log \left(\frac{3+\sqrt{5}}{2}\right)$.

\subsection{Question by Lanneau and Thiffeault}

Let $k$ and $\ell$ be integers such that $0<\ell<k$. We consider the following fibered classes $\operatorname{in} \operatorname{int}\left(C_{\Delta}\right)$ :

$$
\begin{gathered}
(2 k \pm \ell, 2 k \pm 2 \ell, k \pm 2 \ell) \in S_{\beta}\left(\frac{3}{-2}\right), \\
(k, 2 k \pm 2 \ell, \pm \ell) \in S_{\beta}\left(\frac{1}{-2}\right), \\
(k \pm \ell, k \mp \ell,-k) \in S_{\gamma}(2) .
\end{gathered}
$$

By using the Teichmüller polynomial (1-2), we see that the dilatation of each fibered class above is equal to the largest real root $\lambda_{(k, \ell)}$ of the following Lanneau-Thiffeault polynomial:

$$
f_{(k, \ell)}(t)=t^{2 k}-t^{k+\ell}-t^{k}-t^{k-\ell}+1
$$

(note that $f_{(k, \ell)}(t)$ is a common factor of $f_{(2 k \pm \ell, 2 k \pm 2 \ell, k \pm 2 \ell)}(t), f_{(k, 2 k \pm 2 \ell, \pm \ell)}(t)$ and $\left.f_{(k \pm \ell, k \mp \ell,-k)}(t)\right)$.

It is known that $\delta_{2}^{+}=\lambda_{(2,1)}, \delta_{4}^{+}=\lambda_{(4,1)}, \delta_{6}^{+} \geq \lambda_{(6,1)}, \delta_{8}^{+}=\lambda_{(8,1)}$; see $[35 ; 21 ; 14]$. Motivated by these results, Lanneau and Thiffeault asked the following.

Question 1.9 [21] For $g$ even, is $\delta_{g}^{+}$equal to $\lambda_{(g, 1)}$ ?

We consider Question 1.9 in the set $\widehat{\mathcal{M}}^{+}$. The results in this paper imply that there exists a gap between $\widehat{\delta}_{g}^{+}$and $\lambda_{(g, 1)}$ for large $g$ such that $g \equiv 0(\bmod 6)$. 


\section{Theorem 1.10}

(1) We fix any $\epsilon>0$ so that $1.97475-\epsilon>2 \log \left(\frac{3+\sqrt{5}}{2}\right)$. For large $g$ such that $g \equiv 0(\bmod 6)$, we have $\hat{\delta}_{g}^{+}>\lambda_{(g, 1)}$ and

$$
\left|\chi\left(\Sigma_{g}\right)\right| \log \hat{\delta}_{g}^{+}>1.97475-\epsilon>2 \log \left(\frac{3+\sqrt{5}}{2}\right) .
$$

(2) We have $\hat{\delta}_{g}^{+}=\lambda_{(g, 1)}$ for large $g$ such that $g \equiv 2,4(\bmod 6)$.

\subsection{Idea of proofs and conjectures}

This subsection describes the outline of the proof of Theorem 1.4. (The proof of Theorem 1.5 is similar.) First, let us recall the approach to (1-1) by Hironaka [14], Aaber and Dunfield [1] and Kin and Takasawa [19]. Take a particular single 2-cusped hyperbolic fibered 3-manifold $M$ (which is either $N\left(\frac{1}{-2}\right), N\left(\frac{3}{-2}\right)$ or $N(2)$.) Compute the Teichmüller polynomial $P_{\Omega}$ and $\min \operatorname{Ent}(M, \Omega)$ (which equals $2 \log ((3+\sqrt{5}) / 2)$ in this case) for a fibered face $\Omega$ of $M$. Then determine the topological type of each fiber $F$ such that $[F] \in \operatorname{int}\left(C_{\Omega}\right)$. We can find a fiber $F_{g}$ of genus $g$ for large $g$ which enjoys the following. The ray of $\left[F_{g}\right]$ goes to the ray whose normalized entropy Ent achieves $\min \operatorname{Ent}(M, \Omega)$ as $g$ goes to $\infty$. (Then $\operatorname{Ent}\left(\left[F_{g}\right]\right)$ goes to $\min \operatorname{Ent}(M, \Omega)$ as $g$ goes to $\infty$.) Moreover, the number of boundary components of $F_{g}$ is bounded by some constant. Finally check that the stable foliation for the monodromy of the fibration associated to $\left[F_{g}\right]$ satisfies that each boundary component of $F_{g}$ has no 1 prong. Then we obtain the equality in Theorem 1.3 which implies (1-1).

Compared to the above approach, a difficulty for the proof of Theorem 1.4 is that for each $r \in \mathcal{H} y p \backslash\{1\}$, the manifold $N(r)$ has a fiber of arbitrarily large genus. Because of this, it is not clear which manifold $N(r)$ we should look in. Thus it is not a straightforward task to identify a primitive fibered class $a_{g} \in H_{2}(N, \partial N)$ such that $\phi_{a_{g}} \in \mathcal{M}$ and $\hat{\delta}_{g}$ is achieved by $\widehat{\phi}_{a_{g}} \in \widehat{\mathcal{M}} \cap \operatorname{Mod}\left(\Sigma_{g}\right)$. Also it is not obvious at all that one of the boundary slopes of $a_{g}$ becomes a constant for large $g$. (As we will see, one of the boundary slopes of $a_{g}$ must be in $\left\{-4, \frac{3}{-2}, \frac{1}{-2}, 2\right\}$ for large $g$.) The key observation to prove Theorem 1.4 is

Theorem 1.11 For $r \in \mathcal{H} y p$, let $\Omega$ be any fibered face of $N(r)$ which enjoys the following.

(*) Let $a \in S_{\beta}(r)$ be a primitive fibered class of $N$ such that $\bar{a} \in \operatorname{int}\left(C_{\Omega}\right)$. Let $\Phi_{a}: F_{a} \rightarrow F_{a}$ be the monodromy of the fibration associated to $a$. Then the stable foliation $\mathcal{F}_{a}$ of $\Phi_{a}$ has the property such that any boundary component of $F_{a}$ lying on $T_{\beta}$ has no 1 prong. 
Then:

(1) $\min \operatorname{Ent}(N(1), \Omega)=2 \log \delta\left(D_{4}\right) \approx 1.6628$.

(2) If $r=-4, \frac{3}{-2}, \frac{1}{-2}$, or 2 , then we have

$$
\min \operatorname{Ent}(N(r), \Omega)=2 \log \delta\left(D_{3}\right)=2 \log \left(\frac{3+\sqrt{5}}{2}\right) \approx 1.9248
$$

(3) If $r \neq-4, \frac{3}{-2}, \frac{1}{-2}, 1$, or 2 , then we have $\min \operatorname{Ent}(N(r), \Omega)>1.97475$.

We remark here that for any $r \in \mathcal{H} y p$, there exists $\Omega$ having the condition (*) (Proposition 2.24). Thus, there are no slopes $r$ for which we cannot apply Theorem 1.11. Also, since the three claims in Theorem 1.11 cover all slopes $r \in \mathcal{H} y p$, the conclusions we could draw from Theorem 1.11 are expected to be fairly sharp.

To see this, consider the set of pairs

$$
\mathcal{D}=\{(N(r), \Omega) \mid r \in \mathcal{H} y p, \Omega \text { is a fibered face of } N(r) \text { with }(*)\} .
$$

Theorem 1.11 shows for instance that there exist a minimum and a second minimum of

$$
\{\min \operatorname{Ent}(N(r), \Omega) \mid(N(r), \Omega) \in \mathcal{D}\}
$$

and they are $2 \log \delta\left(D_{4}\right)$ and $2 \log \delta\left(D_{3}\right)$ respectively. The minimum is attained only by $N(1)$ and the second minimum is attained by $N(r)$ for $r \in\left\{-4, \frac{3}{-2}, \frac{1}{-2}, 2\right\}$. Furthermore, if $r \notin\left\{-4, \frac{3}{-2}, \frac{1}{-2}, 1,2\right\}$, then Theorem 1.11(3) says $\min \operatorname{Ent}(N(r), \Omega)$ is greater than the second minimum with a uniform gap.

The condition ( $*$ ) on one boundary component, $T_{\beta}$, of $N$ is a weaker version of the condition (1-3) on all three boundary components. If $\Omega$ enjoys (*), then the dilatation of $a \in S_{\beta}(r)$ for $N$ equals the dilatation of $\bar{a}$ for $N(r)$. Thus one can compute the dilatation of $\bar{a}$ by using the Teichmüller polynomial of $N$. In Section 2.2, we shall see that the entropy function for $N$ has symmetries. This property together with the strict concavity of 1 /ent works well in the proof of Theorem 1.11 .

Outline of the proof of Theorem 1.4 It is known that $N(-4) \simeq N\left(\frac{3}{-2}\right)$ and $N(1)$ is isomorphic to the Whitehead link exterior; see [23]. Recall that $a_{g}$ is a primitive fibered class of $H_{2}(N, \partial N)$ such that $\phi_{a_{g}} \in \mathcal{M}$ and $\hat{\delta}_{g}$ is achieved by $\widehat{\phi}_{a_{g}} \in \widehat{\mathcal{M}} \cap \operatorname{Mod}\left(\Sigma_{g}\right)$. There exists such a fibered class $a_{g}$ for any $g \geq 3$. (In fact, the existence of the fiber of the fibration of genus $g$ for any $g \geq 3$ is guaranteed by Theorem 1.3. One sees that the monodromy of this fibration is in the set $\mathcal{M}$, by [19, Lemma 4.7].)

Since we know from the computation that $N(1)$ has no fiber of genus greater than $1, a_{g}$ does not have a boundary slope 1 for $g \geq 2$. On the other hand, each of three manifolds $N(-4) \simeq N\left(\frac{3}{-2}\right), N\left(\frac{1}{-2}\right)$ and $N(2)$ has a fiber of genus $g$ for large $g$. 
Now, if we fill two other cusps of $N(r)$, the normalized entropy of $\widehat{\phi}_{a_{g}}$ decreases from that of $\phi_{a_{g}}$ and we have to consider its defect. We will show that the set of normalized entropies of monodromies of the fibrations on the closed manifolds, obtained from $N$ by Dehn filling all cusps along the slopes not in $\left\{-4, \frac{3}{-2}, \frac{1}{-2}, 2\right\}$, have no accumulation values less than or equal to $2 \log ((3+\sqrt{5}) / 2)$. Thus, one sees that $a_{g}$ has to have a boundary slope in $\left\{-4, \frac{3}{-2}, \frac{1}{-2}, 2\right\}$ eventually. Moreover the set of normalized entropies of the monodromies of the fibrations on the closed manifolds obtained from $N$ by Dehn filling all cusps along the slopes, one of which is in $\left\{-4, \frac{3}{-2}, \frac{1}{-2}, 2\right\}$, have no accumulation values less than $2 \log ((3+\sqrt{5}) / 2)$. This together with Theorem 1.3 implies Theorem 1.4(1).

The proof of Theorem 1.4(1) together with a claim in [19] leads to Theorem 1.4(2). For more details of the proofs, see Sections 3.2 and 3.3.

Based on the study of the magic manifold above, we propose conjectures. (The first half of Conjecture 1.12(1),(2) is also stated in [14, Question 1.12].)

Conjecture 1.12 (1) We have $\lim _{g \rightarrow \infty} g \log \delta_{g}=\log (3+\sqrt{5} / 2)$. For large $g, \delta_{g}$ is achieved by the monodromy of some $\Sigma_{g}$-bundle over the circle obtained from either $N\left(\frac{3}{-2}\right)$ or $N\left(\frac{1}{-2}\right)$ by Dehn filling both cusps.

(2) We have

$$
\lim _{g \neq 0(\bmod 6)} g \log \delta_{g}^{+}=\log \left(\frac{3+\sqrt{5}}{2}\right) .
$$

For large $g$ such that $g \not \equiv 0(\bmod 6), \delta_{g}^{+}$is achieved by the monodromy of some $\Sigma_{g}$-bundle over the circle obtained from $N\left(\frac{3}{-2}\right)$ or $N\left(\frac{1}{-2}\right)$ by Dehn filling both cusps.

Conjecture 1.13 We have $\lim _{n \rightarrow \infty} n \log \delta_{1, n}=2 \log \delta\left(D_{4}\right)$. For large $n, \delta_{1, n}$ is achieved by the monodromy of a fibration on $N(1)$.

\subsection{Organization of the paper}

In Section 2, first we describe properties of the entropy function for $N$. Next we construct the Thurston norm ball of $N(r)$. Finally we discuss the Thurston norm equivalence and entropy equivalence on the manifolds $N(r)$. In Section 3 we prove main results. In Section 4 we exhibit the computation of min Ent for some manifolds $N(r)$ which appeared in Gabai, Meyerhoff and Milley's work (Theorem 4.1, Table 2). We also exhibit the normalized entropy of the monodromy of a fibration on each 1-cusped hyperbolic fibered 3-manifold with volume at most 2.848 . 
Acknowledgments We would like to thank the referee for the careful reading of the paper and for valuable comments and suggestions. Due to the referee's efforts, the exposition of the paper has improved greatly.

The first author is partially supported by Grant-in-Aid for Young Scientists (B) (number 20740031), MEXT, Japan, and the second author is partially supported by Grant-in-Aid (A) (number 22244004), JSPS, Japan.

\section{Magic manifold}

\subsection{Fibered face}

Recall that $\Delta$ is the fibered face of $N$ as in Section 1.4. The open face $\operatorname{int}(\Delta)$ is written by

$$
\operatorname{int}(\Delta)=\{(x, y, z) \mid x+y-z=1, x>0, y>0, x>z, y>z\} .
$$

The Thurston norm of $(x, y, z) \in \operatorname{int}\left(C_{\Delta}\right)$ is given by $x+y-z$. We recall some formulas in Lemmas 2.1 and 2.2. Lemma 2.2 tells us the singularity data of the stable foliation $\mathcal{F}_{a}$ for a primitive fibered class $a \in \operatorname{int}\left(C_{\Delta}\right)$. First of all, we explain that one can compute the dilatation $\lambda(a)$ and the singularity data of the stable foliation $\mathcal{F}_{a}$ for any primitive fibered class $a \in H_{2}(N, \partial N)$ by using the symmetries of $H_{2}(N, \partial N)$.

We consider a homeomorphism (in fact, a rotation map)

$$
h:\left(S^{3}, \mathcal{C}_{3}\right) \rightarrow\left(S^{3}, \mathcal{C}_{3}\right)
$$

which sends $K_{\alpha}, K_{\beta}, K_{\gamma}$ to $K_{\beta}, K_{\gamma}, K_{\alpha}$ respectively; see Figure 2(right). Then $h$ induces the isomorphism $h_{*}: H_{2}(N, \partial N) \rightarrow H_{2}(N, \partial N)$ of order 3 which sends $\alpha, \beta, \gamma$ to $\beta, \gamma, \alpha$ respectively.

Let us pick the two fibered faces $\Delta_{1}$ with the vertices $(0,0,1),(1,1,1),(1,0,0)$, $(0,-1,0)$ and $\Delta_{2}$ with the vertices $(0,1,0),(1,1,1),(0,0,1)$ and $(-1,0,0)$; see Figure 2(left). We denote the opposite fibered faces of $\Delta, \Delta_{1}, \Delta_{2}$ by $\Delta^{\prime}, \Delta_{1}^{\prime}, \Delta_{2}^{\prime}$ respectively. Consider the set

$$
\operatorname{Int} C=\bigcup_{\widehat{\Delta}} \operatorname{int}\left(C_{\widehat{\Delta}}\right)
$$


where $\widehat{\Delta}$ is taken over all fibered face of $N$. We define the map $\sigma: \operatorname{Int} C \rightarrow \operatorname{int}\left(C_{\Delta}\right)$ as follows. For $a=(x, y, z) \in \operatorname{Int} C$,

$$
\begin{aligned}
& \sigma(a)=a \text { if } a \in \operatorname{int}\left(C_{\Delta}\right), \\
& \sigma(a)=h_{*}(a)=(z, x, y) \text { if } a \in \operatorname{int}\left(C_{\Delta_{1}}\right), \\
& \sigma(a)=\left(h^{2}\right)_{*}(a)=(y, z, x) \text { if } a \in \operatorname{int}\left(C_{\Delta_{2}}\right), \\
& \sigma(a)=\sigma(-a) \text { if } a \in \operatorname{int}\left(C_{\Delta^{\prime}}\right) \cup \operatorname{int}\left(C_{\Delta_{1}^{\prime}}\right) \cup \operatorname{int}\left(C_{\Delta_{2}^{\prime}}\right),
\end{aligned}
$$

where $h^{2}=h \circ h$, and $\left(h^{2}\right)_{*}: H_{2}(N, \partial N) \rightarrow H_{2}(N, \partial N)$ is the isomorphism induced from $h^{2}$. Clearly, $a \in H_{2}(N, \partial N)$ is a fibered class if and only if $-a \in H_{2}(N, \partial N)$ is a fibered class. In this case the inverse $\left(\Phi_{a}\right)^{-1}$ of the monodromy $\Phi_{a}$ of the fibration on $N$ associated to $a$ is isotopic to the monodromy $\Phi_{-a}$ of the fibration on $N$ associated to $-a$. In particular $\lambda(a)=\lambda(-a)$. Moreover the singularity datum of $\mathcal{F}_{a}$ and $\mathcal{F}_{-a}$ are the same.

Let us assume $a$ is a primitive fibered class such that $a \in \operatorname{int}\left(C_{\Delta_{1}}\right)\left(\right.$ resp. $a \in \operatorname{int}\left(C_{\Delta_{2}}\right)$ ). Then two fibered classes $a$ and $\sigma(a) \in \operatorname{int}\left(C_{\Delta}\right)$ have the fibres $F_{a}$ and $F_{\sigma(a)}$ with the same topology, and the monodromies $\Phi_{a}$ and $\Phi_{\sigma(a)}$ are conjugate. This is because the isomorphism $h_{*}$ (resp. $\left.\left(h^{2}\right)_{*}\right)$ is coming from the homeomorphism on the pair $\left(S^{3}, \mathcal{C}_{3}\right)$. In particular $\lambda(a)=\lambda(\sigma(a))$, and $\lambda(a)$ is the largest real root of $f_{(z, x, y)}(t)$ (resp. $\left.f_{(y, z, x)}(t)\right)$; see (1-2). Notice that the conjugacy homeomorphism $g: F_{a} \rightarrow F_{\sigma(a)}$ between $\Phi_{a}: F_{a} \rightarrow F_{a}$ and $\Phi_{\sigma(a)}: F_{\sigma(a)} \rightarrow F_{\sigma(a)}$ permutes the boundary components of the fiber. More precisely, $g$ maps the boundary components of $F_{a}$ which lie on $T_{\alpha}, T_{\beta}, T_{\gamma}$ to the boundary components of $F_{\sigma(a)}$ which lie on $T_{\beta}, T_{\gamma}, T_{\alpha}$ (resp. $T_{\gamma}, T_{\alpha}, T_{\beta}$ ). Thus, to apply Lemma 2.2 below for such a primitive fibered class $a$ in $\operatorname{int}\left(C_{\Delta_{1}}\right)$ (resp. $\operatorname{int}\left(C_{\Delta_{2}}\right)$ ), first apply the lemma for $\sigma(a) \in \operatorname{int}\left(C_{\Delta}\right)$. Then translate the claim into the one for the fibered class $a$ by permuting the boundary components of the fiber.

Lemma 2.1 Let $a=(x, y, z)$ be a primitive fibered class in $H_{2}(N, \partial N)$. Then $\sharp\left(\partial F_{a}\right)$ equals

$$
\operatorname{gcd}(x, y+z)+\operatorname{gcd}(y, z+x)+\operatorname{gcd}(z, x+y),
$$

where $\operatorname{gcd}(0, w)$ is defined by $|w|$. More precisely,

$$
\sharp\left(\partial_{\alpha} F_{a}\right)=\operatorname{gcd}(x, y+z), \quad \sharp\left(\partial_{\beta} F_{a}\right)=\operatorname{gcd}(y, z+x), \quad \sharp\left(\partial_{\gamma} F_{a}\right)=\operatorname{gcd}(z, x+y) .
$$

Proof The proof in the case $a \in \operatorname{int}\left(C_{\Delta}\right)$ can be found in [18, Lemma 3.1]. Because of the symmetries of $H_{2}(N, \partial N)$, the formula for primitive fibered classes over $\Delta$ can be extended to any primitive fibered classes in $H_{2}(N, \partial N)$. 
Lemma 2.2 [19, Lemma 3.1] Let $a=(x, y, z)$ be a primitive fibered class in $\operatorname{int}\left(C_{\Delta}\right)$. The stable foliation $\mathcal{F}_{a}$ of the monodromy $\Phi_{a}$ has the property such that each component of $\partial_{\alpha} F_{a}, \partial_{\beta} F_{a}$ and $\partial_{\gamma} F_{a}$ has $x / \operatorname{gcd}(x, y+z)$ prongs, $y / \operatorname{gcd}(y, x+z)$ prongs and $(x+y-2 z) / \operatorname{gcd}(z, x+y)$ prongs respectively. Moreover $\mathcal{F}_{a}$ does not have singularities in the interior of $F_{a}$.

For a rational class $a=(x, y, z) \in H_{2}(N, \partial N ; \mathbb{R})$, define $\frac{p_{\alpha}(a)}{q_{\alpha}(a)}, \frac{p_{\beta}(a)}{q_{\beta}(a)}, \frac{p_{\gamma}(a)}{q_{\gamma}(a)}$ as follows:

$$
\operatorname{slope}(a)=\left(b_{\alpha}(a), b_{\beta}(a), b_{\gamma}(a)\right)=\left(\frac{p_{\alpha}(a)}{q_{\alpha}(a)}, \frac{p_{\beta}(a)}{q_{\beta}(a)}, \frac{p_{\gamma}(a)}{q_{\gamma}(a)}\right),
$$

where $\frac{p_{\alpha}(a)}{q_{\alpha}(a)}, \frac{p_{\beta}(a)}{q_{\beta}(a)}, \frac{p_{\gamma}(a)}{q_{\gamma}(a)}$ are irreducible forms so that $p_{\alpha}(a), p_{\beta}(a), p_{\gamma}(a) \in \mathbb{N}$.

Remark 2.3 Suppose that a rational class $a=(x, y, z)$ is an element of $\operatorname{int}\left(C_{\Delta}\right)$. Then $x>0, y>0, x>z$ and $y>z$ from (2-1). Thus if $z \neq 0$ then $b_{\gamma}(a) \in(-\infty,-2)$ or $b_{\gamma}(a) \in(0, \infty)$. In particular $p_{\gamma}(a)+2 q_{\gamma}(a)>0$ and $p_{\gamma}(a)+q_{\gamma}(a)>0$.

\subsection{Entropy function with symmetries}

In this subsection we will see that the entropy function for $N$ possesses symmetries. Some claims given here play an important role in the proof of Theorem 1.11.

Before we state Lemma 2.4, we note that when $(x, y, z)$ is a primitive fibered class in $\operatorname{int}\left(C_{\Delta}\right)$, then $(y, x, z)$ is also a primitive fibered class in $\operatorname{int}\left(C_{\Delta}\right)$. The topological types of the fibers $F_{(x, y, z)}$ and $F_{(y, x, z)}$ are the same by Lemma 2.1.

Lemma 2.4 Let $(x, y, z)$ be a primitive fibered class in $\operatorname{int}\left(C_{\Delta}\right)$. Then the inverse $\left(\Phi_{(x, y, z)}\right)^{-1}$ of the monodromy $\Phi_{(x, y, z)}: F_{(x, y, z)} \rightarrow F_{(x, y, z)}$ of the fibration on $N$ associated to $(x, y, z)$ is conjugate to the monodromy $\Phi_{(y, x, z)}: F_{(y, x, z)} \rightarrow F_{(y, x, z)}$ of the fibration on $N$ associated to $(y, x, z) \in \operatorname{int}\left(C_{\Delta}\right)$. In particular $\lambda_{(x, y, z)}=\lambda_{(y, x, z)}$.

Proof Let us denote by $\mathcal{C}_{3}^{-}$the 3 chain link such that the orientation of each component is opposite to each one for $\mathcal{C}_{3}$. We denote the components of $\mathcal{C}_{3}^{-}$by $K_{\alpha}^{-}, K_{\beta}^{-}$ and $K_{\gamma}^{-}$. There exists a homeomorphism $i_{\gamma}:\left(S^{3}, \mathcal{C}_{3}\right) \rightarrow\left(S^{3}, \mathcal{C}_{3}^{-}\right)$which sends $K_{\alpha}, K_{\beta}, K_{\gamma}$ to $K_{\beta}^{-}, K_{\alpha}^{-}, K_{\gamma}^{-}$respectively. Then $i_{\gamma}$ induces the isomorphism $\left(i_{\gamma}\right)_{*}: H_{2}(N, \partial N) \rightarrow H_{2}(N, \partial N)$ which sends $\alpha, \beta, \gamma$ to $-\beta,-\alpha,-\gamma$ respectively. If we take $a=(x, y, z) \in \operatorname{int}\left(C_{\Delta}\right)$, then $\left(i_{\gamma}\right)_{*}(a)=(-y,-x,-z) \in \operatorname{int}\left(C_{\Delta^{\prime}}\right)$. Since $\left(i_{\gamma}\right)_{*}$ is induced by the homeomorphism $i_{\gamma}$, the monodromies $\Phi_{a}$ and $\Phi_{\left(i_{\gamma}\right)_{*}(a)}$ must be conjugate. (Hence $\Phi_{a}^{-1}$ and $\left(\Phi_{\left(i_{\gamma}\right)_{*}(a)}\right)^{-1}$ are conjugate.) On the other hand, $\left(\Phi_{\left(i_{\gamma}\right)_{*}(a)}\right)^{-1}$ is isotopic to the monodromy $\Phi_{-\left(i_{\gamma}\right)_{*}(a)}$ of the fibration on $N$ associated to $-\left(i_{\gamma}\right)_{*}(a)=(y, x, z) \in \operatorname{int}\left(C_{\Delta}\right)$. Thus $\left(\Phi_{(x, y, z)}\right)^{-1}$ and $\Phi_{(y, x, z)}$ are conjugate. 
Observe that if we have $(x, y, z) \in \operatorname{int}\left(C_{\Delta}\right)$, then $(y-z, y, y-x),(y-z, x-z,-z)$, $(x, x-z, x-y) \in \operatorname{int}\left(C_{\Delta}\right)$. These four classes have the same Thurston norm.

Lemma 2.5 The four classes $(x, y, z),(y-z, y, y-x),(y-z, x-z,-z)$ and $(x, x-z, x-y)$ of $\operatorname{int}\left(C_{\Delta}\right)$ have the same entropy.

Proof One sees that $f_{(y-z, y, y-x)}(t), f_{(y-z, x-z,-z)}(t)$ and $f_{(x, x-z, x-y)}(t)$ are equal to the same polynomial $f_{(x, y, z)}(t)$.

Remark 2.6 If $(x, y, z)$ is a primitive fibered class in $\operatorname{int}\left(C_{\Delta}\right)$, then the other three classes in Lemma 2.5 are also primitive. Although these classes have the same Thurston norm, the topological types of their minimal representatives may be different.

Let $(x, y, z) \in \Delta$. Since $x+y-z=1$, one may represent $(x, y, z)$ without $z$. Let us denote the class $(x, y, z)$ by $[x, y]$. Then the open face $\operatorname{int}(\Delta)$ can be written by

$$
\operatorname{int}(\Delta)=\{[x, y] \mid 0<x<1,0<y<1\} ;
$$

see Figure 3. We shall see in Remark 2.8 that this parametrization for the points of $\operatorname{int}(\Delta)$ makes it easy to see the symmetry of the entropy function for $N$. We denote by $\lambda_{[x, y]}$ the dilatation of $[x, y] \in \operatorname{int}(\Delta)$. By Lemma 2.5 one obtains the following.

Corollary 2.7 If $(x, y, z) \in \operatorname{int}\left(C_{\Delta}\right)$, then

$$
\begin{aligned}
& {\left[\frac{x}{x+y-z}, \frac{y}{x+y-z}\right], \quad\left[\frac{y-z}{x+y-z}, \frac{y}{x+y-z}\right],} \\
& {\left[\frac{y-z}{x+y-z}, \frac{x-z}{x+y-z}\right], \quad\left[\frac{x}{x+y-z}, \frac{x-z}{x+y-z}\right],}
\end{aligned}
$$

have the same entropy (see Figure 4).

Remark 2.8 Corollary 2.7 says that any two classes of int $(\Delta)$ having a line symmetry about $x=\frac{1}{2}$ (resp. $y=\frac{1}{2}$ ) have the same entropy. In addition by Lemma 2.4, $\lambda_{(x, y, z)}=\lambda_{(y, x, z)}$ holds for $(x, y, z) \in \operatorname{int}\left(C_{\Delta}\right)$. This implies that any two classes $a=[x, y], \tilde{a} \in[y, x] \in \operatorname{int}(\Delta)$ with a line symmetry about $y=x$ have the same entropy. Putting all things together, one has another line symmetry about $y=-x+1$ for the entropy function of $N$. Thus 8 classes $b_{0}, \tilde{b}_{0}, \ldots, b_{3}, \tilde{b}_{3} \in \operatorname{int}(\Delta)$ as in Figure 4 have the same entropy.

By Corollary 2.7, one obtains the following. 


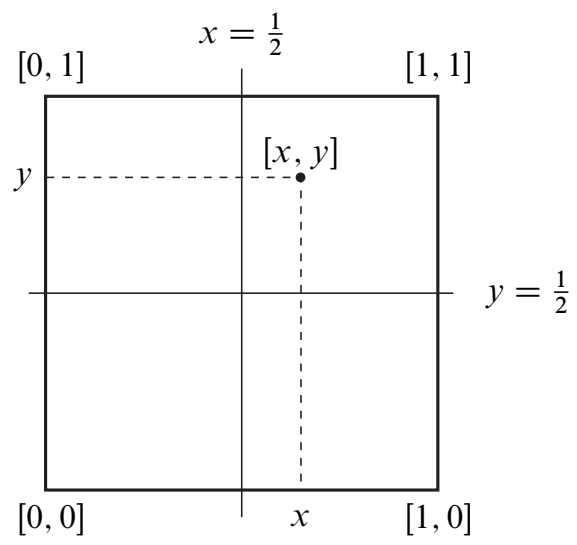

Figure 3: $[x, y] \in \operatorname{int}(\Delta)$
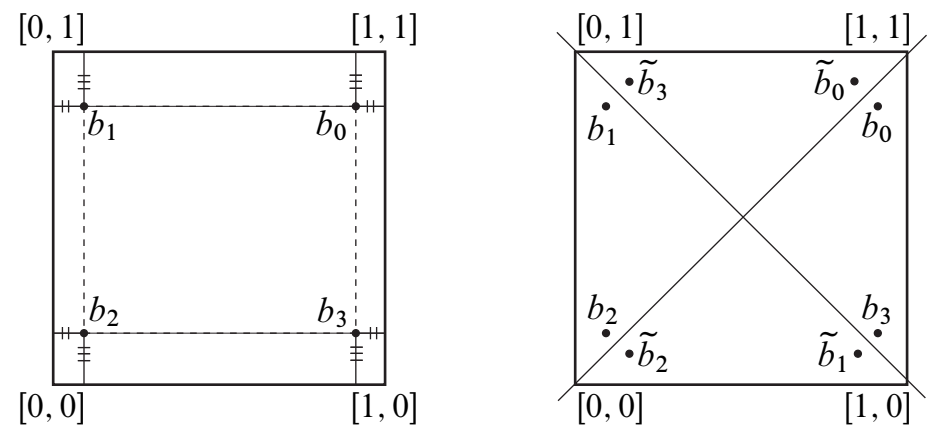

Figure 4: We have that $b_{0}=\left[\frac{x}{x+y-z}, \frac{y}{x+y-z}\right], b_{1}=\left[\frac{y-z}{x+y-z}, \frac{y}{x+y-z}\right]$, $b_{2}=\left[\frac{y-z}{x+y-z}, \frac{x-z}{x+y-z}\right], b_{3}=\left[\frac{x}{x+y-z}, \frac{x-z}{x+y-z}\right] \in \operatorname{int}(\Delta)$ and $\widetilde{b_{i}} \in \operatorname{int}(\Delta)$

Lemma 2.9 Fix $0<x_{0}<1,0<y_{0}<1$ and $0<c<2$.

(1) $\lambda_{\left[x_{0},(1 / 2)-t\right]}=\lambda_{\left[x_{0},(1 / 2)+t\right]}$ for $0 \leq t<(1 / 2)$, and

$$
\lambda_{\left[x_{0}, 1 / 2\right]}=\min \left\{\lambda_{\left[x_{0}, y\right]} \mid 0<y<1\right\} .
$$

(2) $\lambda_{\left[(1 / 2)-t, y_{0}\right]}=\lambda_{\left[(1 / 2)+t, y_{0}\right]}$ for $0 \leq t<\frac{1}{2}$, and

$$
\lambda_{\left[1 / 2, y_{0}\right]}=\min \left\{\lambda_{\left[x, y_{0}\right]} \mid 0<x<1\right\} .
$$

(3) $\lambda_{[(c / 2)+t,(c / 2)-t]}=\lambda_{[(c / 2)-t,(c / 2)+t]}$ for $0 \leq t<1-\frac{c}{2}$, and

$$
\lambda_{[c / 2, c / 2]}=\min \left\{\lambda_{[x, y]} \mid[x, y] \in \operatorname{int}(\Delta), y=-x+c\right\} .
$$

Proof We prove (3). The first equality follows since $\lambda_{[x, y]}=\lambda[y, x]$ for $0<x<1$ and $0<y<1$. The function $1 / \log \lambda$ restricted to the set $\{[x, y] \in \operatorname{int}(\Delta) \mid y=-x+c\}$ is strictly concave. This together with the first equality implies that $\left[\frac{c}{2}, \frac{c}{2}\right]$ reaches a minimum. 
The proofs of (1), (2) are similar to that of (3).

By using Lemma 2.9 one sees that the center $\left[\frac{1}{2}, \frac{1}{2}\right] \in \operatorname{int}(\Delta)$ achieves $\min \operatorname{Ent}(N, \Delta)$. Because of the symmetries of $H_{2}(N, \partial N), \min \operatorname{Ent}(N, \Delta)=\min \operatorname{Ent}(N, \widehat{\Delta})$ holds for any fibered face $\widehat{\Delta}$. Thus one has the following.

Proposition $2.10 \min \operatorname{Ent}(N)=\operatorname{Ent}\left(\left[\frac{1}{2}, \frac{1}{2}\right]\right)=2 \log (2+\sqrt{3}) \approx 2.6339$

By Proposition 2.10, one sees: when $[x, y] \in \operatorname{int}(\Delta)$ such that $[x, y] \neq\left[\frac{1}{2}, \frac{1}{2}\right]$, then

$$
\log \lambda_{[x, y]}>\log \lambda_{[1 / 2,1 / 2]}=2 \log (2+\sqrt{3})>2.633 .
$$

\subsection{Thurston norm of manifolds $N(r)$}

Let $N(r)$ be the manifold obtained from the magic manifold $N$ by Dehn filling the cusp specified by the torus $T_{\beta}$ along the slope $r$, and $D(r)$ an attached solid torus in $N(r)$ so that $\partial D(r)=T_{\beta}$. Consider the exact sequence of the homology group of the triple $(N(r), \partial N(r) \cup D(r), \partial N(r))$ with real coefficients:

$\cdots \longrightarrow H_{2}(N(r), \partial N(r)) \stackrel{j}{\longrightarrow} H_{2}(N(r), \partial N(r) \cup D(r))$

$$
\stackrel{\partial}{\longrightarrow} H_{1}(\partial N(r) \cup D(r), \partial N(r)) \longrightarrow \cdots
$$

The first homomorphism $j$ is injective since $H_{2}(\partial N(r) \cup D(r), \partial N(r))=0$. Also by excision, we have an isomorphism

$$
e: H_{2}(N, \partial N) \longrightarrow H_{2}(N(r), \partial N(r) \cup D(r)) .
$$

Notice that the composition

$$
\partial \circ e: H_{2}(N, \partial N) \longrightarrow H_{1}(\partial N(r) \cup D(r), \partial N(r)) \cong \mathbb{Z}
$$

can be identified with the intersection number for a cycle in $H_{2}(N, \partial N)$ with a slope $r$ on $\partial D(r)=T_{\beta}$.

On the other hand, since the composition of the boundary map with a quotient homomorphism

$$
H_{2}(N, \partial N) \stackrel{\partial}{\longrightarrow} H_{1}(\partial N) \longrightarrow H_{1}(\partial N) / H_{1}\left(T_{\alpha} \cup T_{\gamma}\right) \cong H_{1}\left(T_{\beta}\right)
$$

sends $\alpha$ and $\gamma$ to the minus meridian on $T_{\beta}$ (see Figure 5) and $\beta$ to a longitude, the kernel of $\partial \circ e$ is identified with

$$
S_{\beta}(r)=\left\{(x, y, z) \in H_{2}(N, \partial N) \mid-r y=x+z\right\} .
$$

Thus, we have proved the following. 
Proposition 2.11 Take a slope $r \in \mathbb{Q}$ on a boundary torus for $N$, say $T_{\beta}$. Let $N(r)$ be the manifold obtained from $N$ by Dehn filling the cusp specified by $T_{\beta}$ along the slope $r$. Then there is a natural injection

$$
\iota_{\beta}=e^{-1} \circ j: H_{2}(N(r), \partial N(r)) \longrightarrow H_{2}(N, \partial N)
$$

such that $\operatorname{Im} \iota_{\beta}=S_{\beta}(r)$.

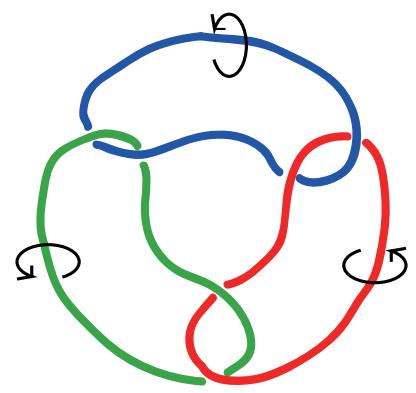

Figure 5: Meridians of the components of $\mathcal{C}_{3}$

For $a=(x, y, z) \in S_{\beta}(r)$, we denote by $\bar{a}=\overline{(x, y, z)}$, the element of $H_{2}(N(r), \partial N(r))$ such that $\iota_{\beta}(\bar{a})=a$. We sometimes denote $N(r)$ by $N_{\beta}(r)$ when we need to specify the cusp which is filled. By using this notation, we may write $\bar{a} \in H_{2}\left(N_{\beta}(r), \partial N_{\beta}(r)\right)$.

Similarly, when $N(r)$ is the manifold obtained from $N$ by Dehn filling the cusp specified by $T_{\alpha}$ or $T_{\gamma}$ along the slope $r$, one has natural injections,

$$
\begin{aligned}
& \iota_{\alpha}: H_{2}(N(r), \partial N(r)) \longrightarrow H_{2}(N, \partial N), \\
& \iota_{\gamma}: H_{2}(N(r), \partial N(r)) \longrightarrow H_{2}(N, \partial N),
\end{aligned}
$$

such that their images are

$$
\begin{aligned}
& S_{\alpha}(r)=\left\{(x, y, z) \in H_{2}(N, \partial N) \mid-r x=y+z\right\}, \\
& S_{\gamma}(r)=\left\{(x, y, z) \in H_{2}(N, \partial N) \mid-r z=x+y\right\} .
\end{aligned}
$$

We also denote by $N_{\alpha}(r)$ or $N_{\gamma}(r)$, the manifold $N(r)$ in this case.

Hereafter we denote the Thurston norm of $N$ by $\|\cdot\|$ and its Thurston norm ball with radius $d$ by $B(d)$. (Hence $U_{N}=B(1)$.) The entropy function and the normalized entropy function of $N$ are denoted by ent and Ent respectively as usual. We also denote the Thurston norm of $N(r)$ by $\|\cdot\|_{r}$ and the Thurston norm ball with radius $d$ by $B_{r}(d)$. The dilatation, entropy function and the normalized entropy function of $N(r)$ are denoted by $\lambda_{r}$, ent $r$ and Ent $r$ respectively. 
Let us define the set $\widehat{B}_{\beta, r}(1)$ to be $\widehat{B}_{\beta, r}(1)=B(1) \cap S_{\beta}(r)$; see Figure 6 . It is parallelogram when $r \in(-2,0)$ (resp. hexagons when $r \in(-\infty,-2) \cup(0, \infty)$ ).
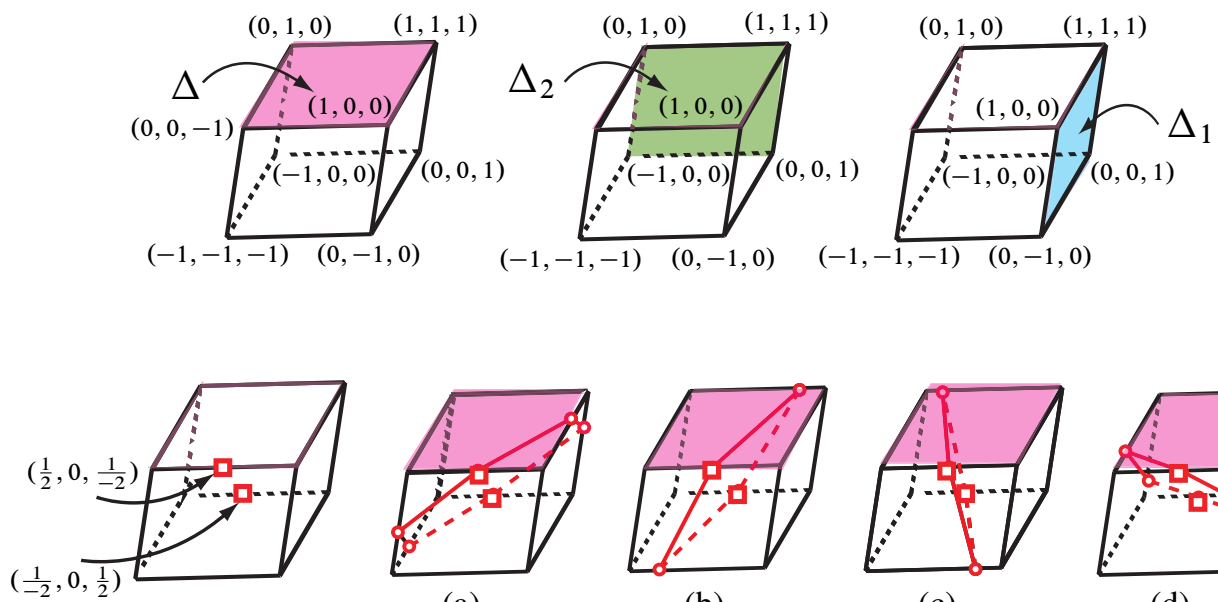

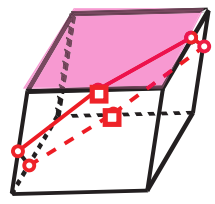

(a)

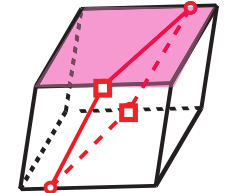

(b)

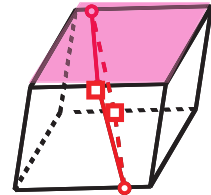

(c)

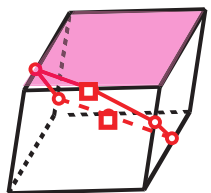

(d)

Figure 6: (Top) fibered faces $\Delta, \Delta_{2}$ and $\Delta_{1}$, (bottom) $\widehat{B}_{\beta, r}(1)$ in the case (a) $r \in(-\infty,-2)$, (b) $r \in(-2,-1)$, (c) $r \in(-1,0)$, (d) $r \in(0, \infty)$

Now we consider the sets $\Delta \cap S_{\alpha}(r), \Delta \cap S_{\beta}(r)$ and $\Delta \cap S_{\gamma}(r)$ for $r \in \mathcal{H} y p$; see Figure 7. Note that $\Delta \cap S_{\gamma}(r) \neq \varnothing$ if and only if $r \in(-\infty,-2) \cup(0, \infty)$; see Remark 2.3.

Lemma 2.12 (1) $\Delta \cap S_{\alpha}(r)$ is a segment $\left\{[x, y] \in \Delta \mid y=\left(\frac{1+r}{-2}\right) x+\frac{1}{2}\right\}$. The set of its endpoints equals:

(i) $\left\{\left[0, \frac{1}{2}\right],\left[\frac{-1}{1+r}, 1\right]\right\}$ when $r \in(-\infty,-2)$.

(ii) $\left\{\left[0, \frac{1}{2}\right],\left[1, \frac{r}{-2}\right]\right\}$ when $r \in(-2,0)$.

(iii) $\left\{\left[0, \frac{1}{2}\right],\left[\frac{1}{1+r}, 0\right]\right\}$ when $r \in(0, \infty)$.

(2) $\Delta \cap S_{\beta}(r)$ is a segment $\left\{[x, y] \in \Delta \mid y=\left(\frac{-2}{1+r}\right) x+\frac{1}{1+r}\right\}$. The set of its endpoints equals:

(i) $\left\{\left[\frac{1}{2}, 0\right],\left[1, \frac{-1}{1+r}\right]\right\}$ when $r \in(-\infty,-2)$.

(ii) $\left\{\left[\frac{1}{2}, 0\right],\left[\frac{r}{-2}, 1\right]\right\}$ when $r \in(-2,0)$.

(iii) $\left\{\left[\frac{1}{2}, 0\right],\left[0, \frac{1}{1+r}\right]\right\}$ when $r \in(0, \infty)$.

(3) $\Delta \cap S_{\gamma}(r)$ is a segment $\left\{[x, y] \in \Delta \mid y=-x+\frac{r}{1+r}\right\}$ when $r \in(-\infty,-2) \cup$ $(0, \infty)$. In this case the set of its endpoints equals:

(i) $\left\{\left[\frac{-1}{1+r}, 1\right],\left[1, \frac{-1}{1+r}\right]\right\}$ when $r \in(-\infty,-2)$.

(ii) $\left\{\left[0, \frac{r}{1+r}\right],\left[\frac{r}{1+r}, 0\right]\right\}$ when $r \in(0, \infty)$. 
Proof We prove the claim (1). Let $a=(x, y, z) \in \Delta \cap S_{\alpha}(r)$. Then $\|a\|=x+y-z=1$ and $-r x=y+z$. Substituting $z=x+y-1$ for $-r x=y+z$, one obtains $y=\left(\frac{1+r}{-2}\right) x+\frac{1}{2}$. It is immediate to check (i), (ii), (iii).

The proofs of (2), (3) are similar to that of (1).
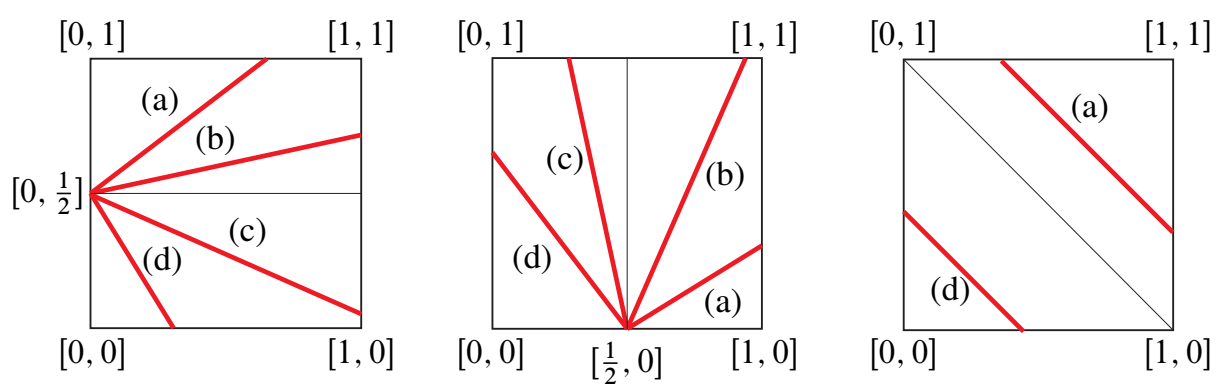

Figure 7: (From left to right) $\Delta \cap S_{\alpha}(r), \Delta \cap S_{\beta}(r), \Delta \cap S_{\gamma}(r)[$ (a) $r \in$ $(-\infty,-2)$, (b) $r \in(-2,-1)$, (c) $r \in(-1,0)$, (d) $r \in(0, \infty)]$

Remark 2.13 We note that $\left(\Delta \cap S_{\alpha}(r)\right) \cup\left(\Delta \cap S_{\beta}(r)\right)$ has a line symmetry about $y=x$.

Lemma 2.14 Suppose that one of the boundary slopes of a rational class of $\mathrm{H}_{2}(\mathrm{~N}, \partial \mathrm{N})$ equals 1 . Then the other two boundary slopes also equal 1 .

Proof Because of the symmetries of $H_{2}(N, \partial N)$, it suffices to suppose that the rational class lives in $\operatorname{int}\left(C_{\Delta}\right)$. By Lemma 2.12, $\Delta \cap S_{\alpha}(1)=\Delta \cap S_{\beta}(1)=\Delta \cap S_{\gamma}(1)$. This leads to the lemma.

We present a formula for the Thurston norm of $N(r)$ by using the Thurston norm of $N$.

Lemma 2.15 Let $p \in \mathbb{N}$ and $q \in \mathbb{Z}$ be coprime such that $p / q \in \mathcal{H} y p$. If $a=(x, y, z) \in S_{\gamma}\left(\frac{p}{q}\right)$, then the Thurston norm of $\bar{a} \in H_{2}\left(N_{\gamma}\left(\frac{p}{q}\right), \partial N_{\gamma}\left(\frac{p}{q}\right)\right)$ equals $\|a\|-\left|\frac{z}{q}\right|$. In particular

$$
\|\bar{a}\|_{p / q}=1-\frac{1}{p+q} \quad \text { if } a \in \Delta \cap S_{\gamma}\left(\frac{p}{q}\right) .
$$


Proof Suppose that $a \in S_{\gamma}\left(\frac{p}{q}\right)$ is an integral class. Then $\|\bar{a}\|_{p / q}$ equals $\|a\|$ minus the number of the boundary components of $F_{a}$ which lie on $T_{\gamma}$, that is

$$
\|\bar{a}\|_{p / q}=\|a\|-\operatorname{gcd}(z, x+y)=\|a\|-\left|\frac{z}{q}\right| .
$$

The Thurston norm $\|\cdot\|_{p / q}$ defined on integral classes admits a unique continuous extension to $H_{2}\left(N\left(\frac{p}{q}\right), \partial N\left(\frac{p}{q}\right) ; \mathbb{R}\right)$. Thus we have that the above formula holds for any class $a \in S_{\gamma}\left(\frac{p}{q}\right)$.

Suppose that $a \in \Delta \cap S_{\gamma}\left(\frac{p}{q}\right)$. Then $p+q>0$. One has $-p z=q(x+y)$ and $\|a\|=x+y-z=1$. Hence $-p z=q(1+z)$, and one obtains $z=\frac{q}{-(p+q)}$. Thus $\|\bar{a}\|_{p / q}=1-\left|\frac{z}{q}\right|=1-\frac{1}{p+q}$.

Similarly, we have the following.

Lemma 2.16 If one fills the cusp of $N$ specified by the torus $T_{\alpha}$ (resp. $T_{\beta}$ ) along the slope $\frac{p}{q}$, then the Thurston norm of $N_{\alpha}\left(\frac{p}{q}\right)$ (resp. $\left.N_{\beta}\left(\frac{p}{q}\right)\right)$ is given by

$$
\begin{aligned}
\|\bar{a}\|_{p / q} & =\|a\|-\left|\frac{x}{q}\right| \text { for } a=(x, y, z) \in S_{\alpha}\left(\frac{p}{q}\right), \\
\left(\text { resp. }\|\bar{a}\|_{p / q}\right. & \left.=\|a\|-\left|\frac{y}{q}\right| \text { for } a=(x, y, z) \in S_{\beta}\left(\frac{p}{q}\right)\right) .
\end{aligned}
$$

\subsection{Thurston norm equivalence on manifolds $N(r)$}

Let $p \in \mathbb{N}$ and $q \in \mathbb{Z}$ be coprime such that $r=\frac{p}{q} \in \mathcal{H} y p$. We shall investigate the shape of the Thurston norm ball of $N(r)=N_{\beta}(r)$.

First we take $\mathfrak{a}_{r}, \mathfrak{b}_{r} \in S_{\beta}(r)$ as follows:

$$
\begin{aligned}
\mathfrak{a}_{r} & =\left(\frac{p+1}{2},-q, \frac{p-1}{2}\right), \mathfrak{b}_{r}=\left(\frac{p-1}{2},-q, \frac{p+1}{2}\right) \text { if } p \text { is odd, } \\
\mathfrak{a}_{r} & =\left(\frac{p}{2}+1,-q, \frac{p}{2}-1\right), \mathfrak{b}_{r}=\left(\frac{p}{2},-q, \frac{p}{2}\right) \text { if } p \text { is even. }
\end{aligned}
$$

Lemma 2.17 The set $\left\{\overline{\mathfrak{a}}_{r}, \overline{\mathfrak{b}}_{r}\right\}$ is a basis of $H_{2}\left(N_{\beta}(r), \partial N_{\beta}(r) ; \mathbb{Z}\right)$.

Proof By Proposition 2.11, $\operatorname{Im} \iota_{\beta}=S_{\beta}(r)$. Thus it is enough to show for any integral class $a=(x, y, z) \in S_{\beta}(r)$, there exist integers $k_{0}, \ell_{0}$ such that $a=k_{0} \mathfrak{a}_{r}+\ell_{0} \mathfrak{b}_{r}$. One has $-p y=q(x+z)$. Since $p$ and $q$ are coprime, there exists an integer $t$ such that $x+z=p t$. Hence $z=p t-x$. Substitute $x+z=p t$ for $-p y=q(x+z)$, then one obtains $(x, y, z)=(x,-q t, p t-x)$. Now let us take $k_{0}=\left(\frac{1-p}{2}\right) t+x$, $\ell_{0}=\left(\frac{1+p}{2}\right) t-x$ if $p$ is odd (resp. $k_{0}=\left(\frac{-p}{2}\right) t+x, \ell_{0}=\left(1+\frac{p}{2}\right) t-x$ if $p$ is even). One can check that $a=k_{0} \mathfrak{a}_{r}+\ell_{0} \mathfrak{b}_{r}$. 
Lemma 2.18_Let $r=\frac{p}{q}$ be as above. The Thurston norm ball of $N(r)$ is described by using $\left(\overline{\mathfrak{a}}_{r}, \overline{\mathfrak{b}}_{r}\right)$ coordinates as follows (see Figure 8 ).

(1) Suppose that $r \in(-\infty,-2)$.

(i) If $|q|(=-q) \neq 1$, then $B_{r}(p+q-1)$ is a hexagon with vertices

$$
\begin{aligned}
& \pm\left(\frac{p+2 q+1}{2}, \frac{p+2 q-1}{-2}\right), \pm\left(\frac{p+2 q-1}{2}, \frac{p+2 q+1}{-2}\right), \\
& \pm\left(\frac{p+q-1}{2}, \frac{p+q-1}{-2}\right) \quad \text { when } p \text { is odd, } \\
& \pm\left(\frac{p+2 q}{2}, \frac{p+2 q-2}{-2}\right), \pm\left(\frac{p+2 q}{2}, \frac{p+2 q+2}{-2}\right), \\
& \pm\left(\frac{p+q-1}{2}, \frac{p+q-1}{-2}\right) \text { when } p \text { is even. }
\end{aligned}
$$

(ii) If $|q|(=-q)=1$, then $B_{r}(p+q-1)$ is a rectangle with vertices

$$
\begin{array}{r} 
\pm\left(\frac{p+2 q+1}{2}, \frac{p+2 q-1}{-2}\right), \pm\left(\frac{p+2 q-1}{2}, \frac{p+2 q+1}{-2}\right) \quad \text { when } p \text { is odd, } \\
\pm\left(\frac{p+2 q}{2}, \frac{p+2 q-2}{-2}\right), \pm\left(\frac{p+2 q}{2}, \frac{p+2 q+2}{-2}\right) \quad \text { when } p \text { is even. }
\end{array}
$$

(2) Suppose that $r \in(-2,0)$. Then $B_{r}(-q)$ is a parallelogram with vertices

$$
\pm\left(\frac{q}{2 q+2}, \frac{q}{2 q+2}\right), \pm\left(\frac{q}{-2}, \frac{q}{2}\right) \text { when } p \text { is odd, }
$$

and $B_{r}(-q-1)$ is a parallelogram with vertices

$$
\pm(0,1), \pm\left(\frac{q+1}{-2}, \frac{q+1}{2}\right) \text { when } p \text { is even. }
$$

(3) Suppose that $r \in(0, \infty)$.

(i) If $|q|(=q) \neq 1$, then $B_{r}(p+q-1)$ is a hexagon with vertices

$\pm\left(\frac{p+1}{2}, \frac{p-1}{-2}\right), \pm\left(\frac{p-1}{2}, \frac{p+1}{-2}\right), \pm\left(\frac{p+q-1}{2}, \frac{p+q-1}{-2}\right)$ when $p$ is odd,

$$
\pm\left(\frac{p}{2}, \frac{p-2}{-2}\right), \pm\left(\frac{p}{2}, \frac{p+2}{-2}\right), \pm\left(\frac{p+q-1}{2}, \frac{p+q-1}{-2}\right) \text { when } p \text { is even. }
$$

(ii) If $|q|(=q)=1$, then $B_{r}(p+q-1)$ is a rectangle with vertices

$$
\begin{array}{r} 
\pm\left(\frac{p+1}{2}, \frac{p-1}{-2}\right), \pm\left(\frac{p-1}{2}, \frac{p+1}{-2}\right) \quad \text { when } p \text { is odd } \\
\pm\left(\frac{p}{2}, \frac{p-2}{-2}\right), \pm\left(\frac{p}{2}, \frac{p+2}{-2}\right) \text { when } p \text { is even. }
\end{array}
$$


Proof Let us consider the classes in $\widehat{B}_{\beta, r}(1)\left(=B(1) \cap S_{\beta}(r)\right)$; see Figure 6 . Then $\|a\|=1$ and $\|\bar{a}\|_{r}=1-\left|\frac{y}{q}\right|$ for all $a=(x, y, z) \in \widehat{B}_{\beta, r}(1)$. To find the Thurston norm ball of $N(r)$, one needs to shear $\widehat{B}_{\beta, r}(1)$ by an appropriate amount depending on the $y$-coordinate of $a$. One can see that the shearing turns the parallelogram/hexagon into another parallelogram/hexagon unless $|q|$ equals 1 . The degeneration of the Thurston norm ball of $N(r)$ occurs when $|q|$ equals 1 . In this case, the shearing makes two sides of the hexagon line up, and the hexagon turns into a rectangle. By using this argument, it is straightforward to verify the lemma.

Note that every top-dimensional face on the boundary of the Thurston norm ball of $N(r)$ is a fibered face for each $r \in \mathcal{H} y p$. Figure 9 illustrates the Thurston norm balls of $N\left(\frac{5}{-2}\right), N\left(\frac{3}{-2}\right), N\left(\frac{2}{-3}\right)$ and $N(1)$.

We now prove that there exist infinitely many Thurston norm equivalent pairs obtained from $N$ by Dehn filling.

Lemma 2.19 Let $p \in \mathbb{N}$ and $q \in \mathbb{Z}$ (resp. $p^{\prime} \in \mathbb{N}$ and $q \in \mathbb{Z}$ ) be coprime such that $r=\frac{p}{q}, r^{\prime}=\frac{p^{\prime}}{q} \in \mathcal{H} y p \cap(-2,0)$. Suppose that either both $p$ and $p^{\prime}$ are odd or both $p$ and $p^{\prime}$ are even. Then $N(r) \underset{\mathrm{T}}{\sim} N\left(r^{\prime}\right)$.

Proof Suppose that $p$ and $p^{\prime}$ are odd. The numerator does not appear in the vertices of $B_{r}(-q)$; see Lemma 2.18(2). The position for the vertices of $B_{r}(-q)$ is the same as that of $B_{r^{\prime}}(-q)$. Thus the natural isomorphism $f: H_{2}(N(r), \partial N(r) ; \mathbb{Z}) \rightarrow$ $H_{2}\left(N\left(r^{\prime}\right), \partial N\left(r^{\prime}\right) ; \mathbb{Z}\right)$ which sends $\overline{\mathfrak{a}}_{r}$ to $\overline{\mathfrak{a}}_{r^{\prime}}$ and $\overline{\mathfrak{b}}_{r}$ to $\overline{\mathfrak{b}}_{r}^{\prime}$ becomes a Thurston norm preserving isomorphism.

The proof in the case $p$ and $p^{\prime}$ are even is similar.

Proposition 2.20 Suppose that both $r,-2-r \in \mathcal{H} y p$. Then $N(r) \underset{\mathrm{T}}{\sim} N(-2-r)$.

Proof Let $p \in \mathbb{N}$ and $q \in \mathbb{Z}$ be coprime such that $r=p / q \in \mathcal{H} y p$. We have shown the claim when $p / q \in(-2,-1)$; see Lemma 2.19. Now suppose that $p / q \in(-\infty,-2)$. Let us set an irreducible form $r^{\prime}=p^{\prime} / q^{\prime}=(p+2 q) /(-q)\left(p^{\prime}=p+2 q \in \mathbb{N}\right)$. By Lemma 2.18(1) and (3), $B_{r^{\prime}}\left(p^{\prime}+q^{\prime}-1\right)$ and $B_{r}(p+q-1)$ are hexagons when $|q| \neq 1$ (resp. rectangle when $|q|=1)$. The position for the vertices of $B_{r^{\prime}}\left(p^{\prime}+q^{\prime}-1\right)$ is the same as that of $B_{r}(p+q-1)$. The Thurston norm balls $B_{r^{\prime}}\left(p^{\prime}+q^{\prime}-1\right)$ and $B_{r}(p+q-1)$ have the same radius, ie, $p^{\prime}+q^{\prime}-1=p+q-1$. Thus the natural isomorphism $f: H_{2}(N(r), \partial N(r) ; \mathbb{Z}) \rightarrow H_{2}\left(N\left(r^{\prime}\right), \partial N\left(r^{\prime}\right) ; \mathbb{Z}\right)$ which sends $\overline{\mathfrak{a}}_{r}$ to $\overline{\mathfrak{a}}_{r^{\prime}}$ and $\overline{\mathfrak{b}}_{r}$ to $\overline{\mathfrak{b}}_{r^{\prime}}$ becomes a Thurston norm preserving isomorphism. 

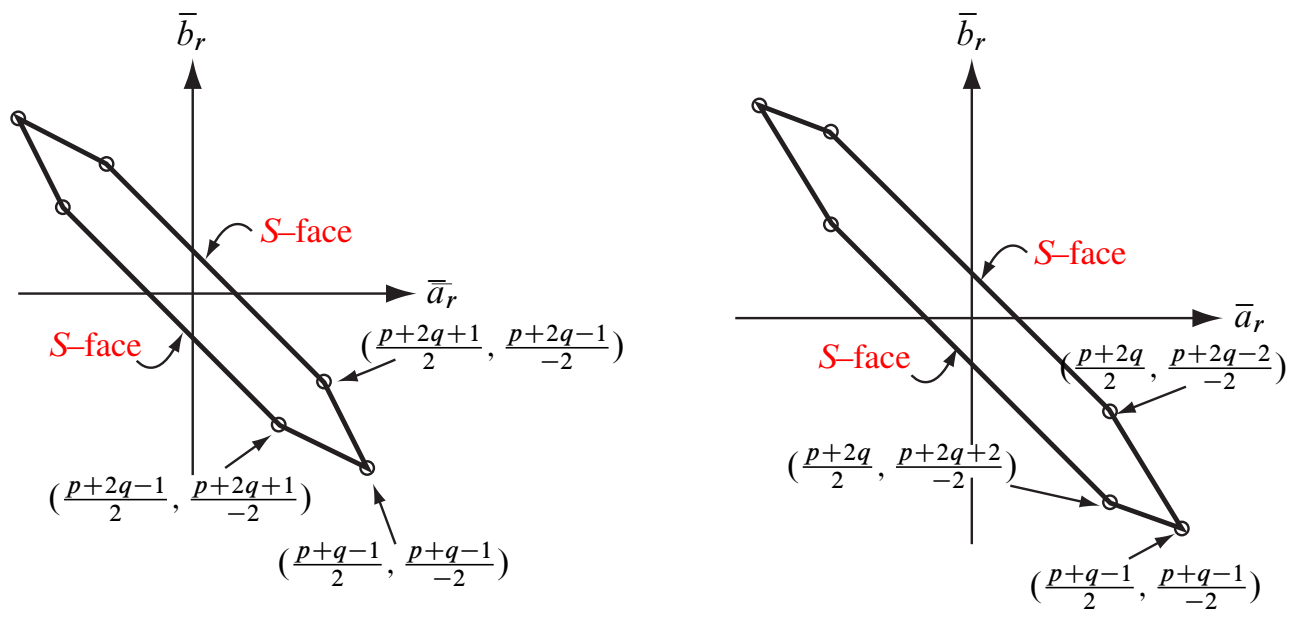

$$
\left(1-\mathrm{i}-\text { odd } \quad B_{r}(p+q-1)\right)
$$

$$
\left(1-\mathrm{i}-\mathrm{even} \quad B_{r}(p+q-1)\right)
$$
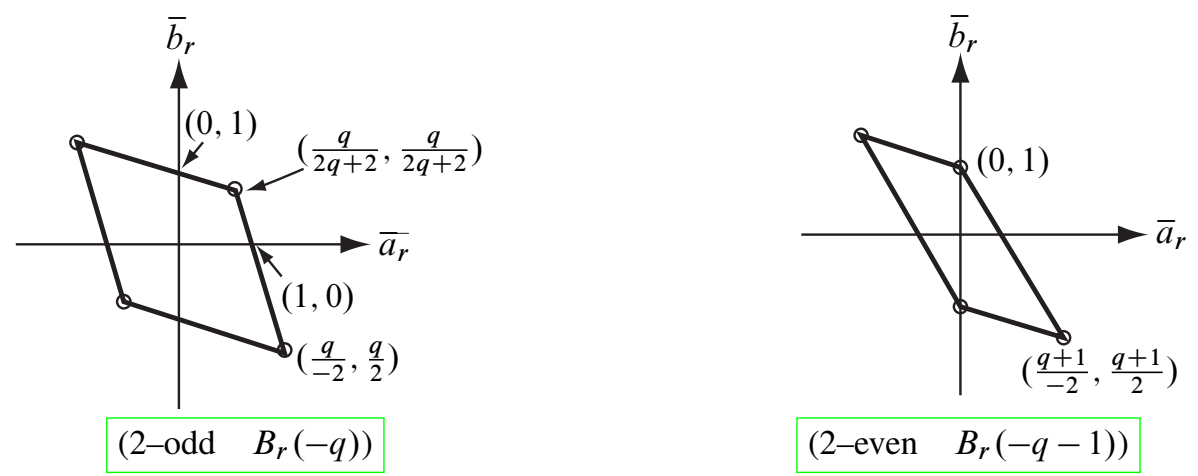

Figure 8: Thurston norm ball $B_{r}(d)$ (with radius $\left.d\right)$ of $N\left(\frac{p}{q}\right) ;(1-\mathrm{i}-$ odd) $p / q \in(-\infty,-2), q \neq 1$ and $p$ is odd; (1-i-even) $p / q \in(-\infty,-2), q \neq 1$ and $p$ is even; (2-odd) $p / q \in(-2,0)$ and $p$ is odd; (2-even) $p / q \in(-2,0)$ and $p$ is even

\subsection{Entropy equivalence on fibered 3-manifolds}

2.5.1 Definition of entropy equivalence Let $(M, \Omega)$ and $\left(M^{\prime}, \Omega^{\prime}\right)$ be pairs of $3-$ manifolds $M, M^{\prime}$ and their fibered faces $\Omega, \Omega^{\prime}$ respectively. Possibly $M \simeq M^{\prime}$. Then $(M, \Omega)$ and $\left(M^{\prime}, \Omega^{\prime}\right)$ are entropy equivalent, denoted by

$$
(M, \Omega) \underset{\mathrm{ent}}{\sim}\left(M^{\prime}, \Omega^{\prime}\right),
$$

if we have that there is a Thurston norm preserving isomorphism $f: H_{2}(M, \partial M ; \mathbb{Z}) \rightarrow$ $H_{2}\left(M^{\prime}, \partial M^{\prime} ; \mathbb{Z}\right)$ satisfying the following: 


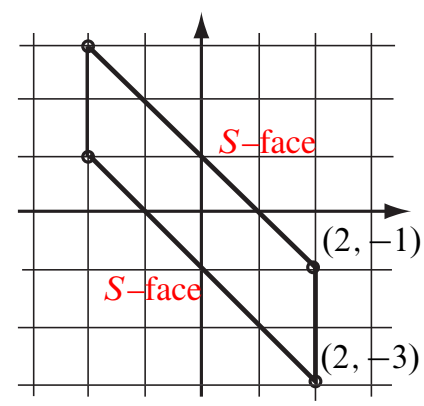

(a) $B_{r}(4)$

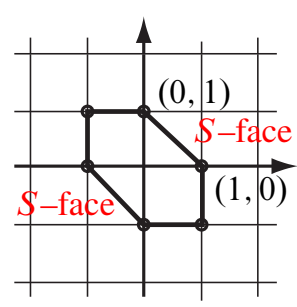

(b) $B_{r}(2)$

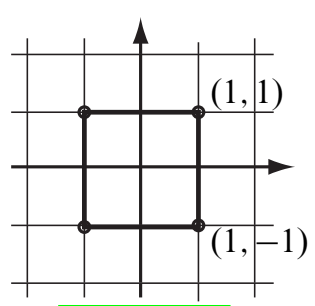

(c) $B_{r}(2)$

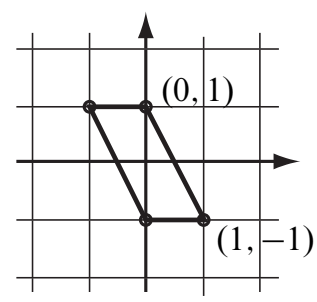

(d) $B_{r}(2)$

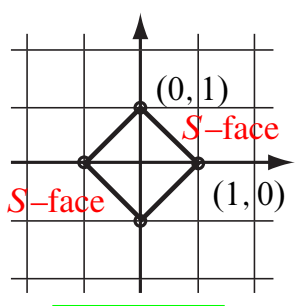

(e) $B_{r}(1)$

Figure 9: Thurston norm ball $B_{r}(d)$ (with radius $d$ ) of $N(r)$ when (a) $r=-6,4$, (b) $r=\frac{5}{-2}, \frac{1}{2}$, (c) $r=\frac{3}{-2}, \frac{1}{-2}$, (d) $r=\frac{4}{-3}, \frac{2}{-3}$, (e) $r=1$.

- $a \in \operatorname{int}\left(C_{\Omega}(\mathbb{Z})\right)$ if and only if $f(a) \in \operatorname{int}\left(C_{\Omega^{\prime}}(\mathbb{Z})\right)$.

- $\operatorname{ent}(a)=\operatorname{ent}(f(a))$ for any $a \in \operatorname{int}\left(C_{\Omega}(\mathbb{Z})\right)$.

The second bullet implies that $\operatorname{ent}(a)=\operatorname{ent}(f(a))$ for any $a \in \operatorname{int}\left(C_{\Omega}\right)$, since we have that ent: $\operatorname{int}\left(C_{\Omega}(\mathbb{Q})\right) \rightarrow \mathbb{R}$ admits a unique continuous extension. Thus if

$$
(M, \Omega) \underset{\mathrm{ent}}{\sim}\left(M^{\prime}, \Omega^{\prime}\right),
$$

then $\min \operatorname{Ent}(M, \Omega)=\min \operatorname{Ent}\left(M^{\prime}, \Omega^{\prime}\right)$.

Here is an obvious example. If a face $\Omega^{\prime}$ of $M$ is opposite to a fibered face $\Omega$, then $\Omega^{\prime}$ is also a fibered face. The pairs $(M, \Omega)$ and $\left(M, \Omega^{\prime}\right)$ are entropy equivalent, because the isomorphism on $H_{2}(M, \partial M ; \mathbb{Z})$ given by $a \mapsto-a$ preserves the Thurston norm and entropy.

Fibered 3-manifolds $M$ and $M^{\prime}$ are entropy equivalent, denoted by

$$
M \underset{\mathrm{ent}}{\sim} M^{\prime}
$$


if we have that there is a Thurston norm preserving isomorphism $f: H_{2}(M, \partial M ; \mathbb{Z}) \rightarrow$ $H_{2}\left(M^{\prime}, \partial M^{\prime} ; \mathbb{Z}\right)$ satisfying the following:

- $a \in H_{2}(M, \partial M ; \mathbb{Z})$ is a fibered class if and only if $f(a) \in H_{2}\left(M^{\prime}, \partial M^{\prime} ; \mathbb{Z}\right)$ is a fibered class.

- Given a fibered face $\Omega$ of $M$, we have $\operatorname{ent}(a)=\operatorname{ent}(f(a))$ for any $a \in \operatorname{int}\left(C_{\Omega}(\mathbb{Z})\right.$ ).

If $M \underset{\text { ent }}{\sim} M^{\prime}$, then $\min \operatorname{Ent}(M)=\min \operatorname{Ent}\left(M^{\prime}\right)$.

2.5.2 Entropy equivalence on manifolds $N(\boldsymbol{r})$ In this subsection, first of all we focus on the set $S_{\beta}(r)$ and the stable foliation $\mathcal{F}_{a}$ for $a \in S_{\beta}(r)$. We compute the number of prongs on each boundary component of $F_{a}$ lying on $T_{\beta}$. We will see this number depends on the slope $r$ and the fibered face $\Omega$ of $N(r)$ with the property $\bar{a} \in \operatorname{int}\left(C_{\Omega}\right)$. Then we discuss the entropy equivalence between $N(r)$ and $N(-2-r)$ when $r,-2-r \in \mathcal{H} y p$.

We begin with the definition of $A$-faces and $S$-faces. They are top-dimensional faces $\Omega$ on the boundary of the Thurston norm ball of $N(r)$ for $r=\frac{p}{q} \in \mathcal{H} y p$.

- Suppose that $|q| \neq 1$. Then $\Omega$ is called an $A$-face if an element of $\partial \Omega$ is equal to $\overline{\alpha-\gamma}$ projectively. Equivalently, $\Omega$ is an $A$-face if an element of $\partial \Omega$ is equal to $\overline{\mathfrak{a}}_{r}-\overline{\mathfrak{b}}_{r}$ projectively. A face $\Omega$ is called an $S$-face if it is not an $A$-face.

- Suppose that $|q|=1$. Then $\Omega$ is called an $A$-face if the interior of the cone over $\Omega$ contains $\overline{\alpha-\gamma}$ projectively. Equivalently, $\Omega$ is an $A$-face if the interior of the cone over $\Omega$ contains $\overline{\mathfrak{a}}_{r}-\overline{\mathfrak{b}}_{r}$ projectively. A face $\Omega$ is called an $S$-face if it is not an $A$-face.

It follows from Lemma 2.18 that every top-dimensional face for $N(r)$ is an $A$-face if $r \in(-2,0)$. When $r \in(-\infty,-2) \cup(0, \infty)$ such that $|q| \neq 1$ (resp. $|q|=1)$, the Thurston norm ball for $N(r)$ is a hexagon (resp. rectangle) having two $S$-faces and four $A$-faces (resp. having two $S$-faces and two $A$-faces); see Figures 8 and 9.

It is worthwhile to point out that the two $S$-faces come from the fibered face $\Delta_{1}$ and its opposite face $\Delta_{1}^{\prime}$ for $N$; see Figure 6(a), (d). Let us turn to the $A$-faces. If we have that $|q| \neq 1$, then the Thurston norm ball of $N(r)$ has four $A$-faces, and they come from the four fibered faces $\Delta, \Delta_{2}$ and their opposite faces $\Delta^{\prime}, \Delta_{2}^{\prime}$ (see Figure 6(a)-(d)). The degeneration of $A$-faces occur when $|q|=1$. In this case, the Thurston norm ball of $N(r)$ has two $A$-faces. One of the $A$-faces comes from the pair $\Delta$ and $\Delta_{2}^{\prime}$. The other $A$-face comes from the pair $\Delta^{\prime}$ and $\Delta_{2}$. This observation leads to the following. 
Lemma 2.21 Let $\sigma: \operatorname{Int} C \rightarrow \operatorname{int}\left(C_{\Delta}\right)$ be the map given in Section 2.1. We take a class $a \in S_{\beta}(r) \cap \operatorname{Int} C$.

(1) If $a \in S_{\beta}(r) \cap\left(\operatorname{int}(\Delta) \cup \operatorname{int}\left(\Delta^{\prime}\right)\right)$, then $\bar{a} \in \operatorname{int}\left(C_{\Omega_{A}}\right) \subset H_{2}\left(N_{\beta}(r), \partial N_{\beta}(r)\right)$ for some $A-$ face, and $\sigma(a) \in \operatorname{int}\left(C_{\Delta}\right) \cap S_{\beta}(r)$.

(2) If $a \in S_{\beta}(r) \cap\left(\operatorname{int}\left(\Delta_{1}\right) \cup \operatorname{int}\left(\Delta_{1}^{\prime}\right)\right)$, then $\bar{a} \in \operatorname{int}\left(C_{\Omega_{S}}\right) \subset H_{2}\left(N_{\beta}(r), \partial N_{\beta}(r)\right)$ for some $S$-face, and $\sigma(a) \in \operatorname{int}\left(C_{\Delta}\right) \cap S_{\gamma}(r)$.

(3) If $a \in S_{\beta}(r) \cap\left(\operatorname{int}\left(\Delta_{2}\right) \cup \operatorname{int}\left(\Delta_{2}^{\prime}\right)\right)$, then $\bar{a} \in \operatorname{int}\left(C_{\Omega_{A}}\right) \subset H_{2}\left(N_{\beta}(r), \partial N_{\beta}(r)\right)$ for some $A$-face, and $\sigma(a) \in \operatorname{int}\left(C_{\Delta}\right) \cap S_{\alpha}(r)$.

Lemma 2.22 Let $r \in \mathcal{H} y p$. Any two $S$-faces of $N(r)$ are entropy equivalent, and any two $A$-faces of $N(r)$ are entropy equivalent.

Proof An $S$-face of $N(r)$ is opposite to the other $S$-face, and hence they are entropy equivalent (see the example after the definition of entropy equivalence). Similarly, if an $A$-face $\Omega$ is opposite side to an $A$-face $\Omega^{\prime}$, then they are entropy equivalent. Thus the proof in the case $r \in \mathbb{Z}$ is done.

We assume that $r=\frac{p}{q} \notin \mathbb{Z}$, ie, $q \neq 1$. We need to show that an $A$-face $\Omega$ is entropy equivalent to an $A$-face $\widehat{\Omega}$ which is not the opposite face $\Omega^{\prime}$. To do this, it is enough to prove that the $A$-face of $N(r)$ coming from $\Delta$, say $\Omega_{A, \Delta}$, and the $A$-face of $N(r)$ coming from $\Delta_{2}$, say $\Omega_{A, \Delta_{2}}$ are entropy equivalent. We first find the Thurston norm preserving isomorphism

$$
f: H_{2}(N(r), \partial N(r) ; \mathbb{Z}) \rightarrow H_{2}(N(r), \partial N(r) ; \mathbb{Z})
$$

which sends $\operatorname{int}\left(C_{\Omega_{A, \Delta_{2}}}(\mathbb{Z})\right)$ to $\operatorname{int}\left(C_{\Omega_{A, \Delta}}(\mathbb{Z})\right)$. We recall the two isomorphisms

$$
\begin{aligned}
\left(h^{2}\right)_{*}: H_{2}(N, \partial N ; \mathbb{Z}) & \rightarrow H_{2}(N, \partial N ; \mathbb{Z}), \\
(x, y, z) & \mapsto(y, z, x), \\
-\left(i_{\gamma}\right)_{*}: H_{2}(N, \partial N ; \mathbb{Z}) & \rightarrow H_{2}(N, \partial N ; \mathbb{Z}), \\
(x, y, z) & \mapsto(y, x, z) ;
\end{aligned}
$$

see the proof of Lemma 2.4. Observe that $\left(h^{2}\right)_{*}\left(S_{\beta}(r)\right)=S_{\alpha}(r)$. This shows that we have the isomorphism

$$
\begin{aligned}
\overline{\left(h^{2}\right)_{*}: H_{2}\left(N_{\beta}(r), \partial N_{\beta}(r) ; \mathbb{Z}\right)} & \rightarrow H_{2}\left(N_{\alpha}(r), \partial N_{\alpha}(r) ; \mathbb{Z}\right), \\
\overline{(x, y, z)} & \mapsto \overline{(y, z, x),}
\end{aligned}
$$


induced from $\left(h^{2}\right)_{*}$. On the other hand, we have $-\left(i_{\gamma}\right)_{*}\left(S_{\alpha}(r)\right)=S_{\beta}(r)$. Thus $-\left(i_{\gamma}\right)_{*}$ induces the isomorphism

$$
\begin{aligned}
\overline{-\left(i_{\gamma}\right)_{*}}: H_{2}\left(N_{\alpha}(r), \partial N_{\alpha}(r) ; \mathbb{Z}\right) & \rightarrow H_{2}\left(N_{\beta}(r), \partial N_{\beta}(r) ; \mathbb{Z}\right), \\
\overline{(x, y, z)} & \mapsto \overline{(y, x, z)} .
\end{aligned}
$$

Let us set $f={\overline{-\left(i_{\gamma}\right)_{*}}}_{*} \overline{\left(h^{2}\right)}$. One sees that $f$ sends $\operatorname{int}\left(C_{\Omega_{A, \Delta_{2}}}(\mathbb{Z})\right)$ to $\operatorname{int}\left(C_{\Omega_{A, \Delta}}(\mathbb{Z})\right)$, because

$$
\begin{aligned}
\left(h^{2}\right)_{*}\left(S_{\beta}(r) \cap \operatorname{int}\left(C_{\Delta_{2}}(\mathbb{Z})\right)\right) & =S_{\alpha}(r) \cap \operatorname{int}\left(C_{\Delta}(\mathbb{Z})\right), \\
-\left(i_{\gamma}\right)_{*}\left(S_{\alpha}(r) \cap \operatorname{int}\left(C_{\Delta}(\mathbb{Z})\right)\right) & =S_{\beta}(r) \cap \operatorname{int}\left(C_{\Delta}(\mathbb{Z})\right) .
\end{aligned}
$$

Then $f$ preserves the Thurston norm, since both ${\overline{\left(h^{2}\right.}}_{*}$ and $\overline{-\left(i_{\gamma}\right)} *$ preserve the Thurston norm by Lemma 2.16.

We now prove that $f$ preserves the entropies on $\operatorname{int}\left(C_{\Omega_{A, \Delta_{2}}}(\mathbb{Z})\right)$. Let $(x, y, z) \in$ $S_{\beta}(r) \cap \operatorname{int}\left(C_{\Delta_{\underline{2}}}(\mathbb{Z})\right)$. Then $\overline{(x, y, z)}$ and $\overline{\left(h^{2}\right)_{*}}(\overline{(x, y, z)})=\overline{(y, z, x)}$ have the same entropy, since $\left(h^{2}\right)_{*}$ is induced from the homeomorphism $h^{2}:\left(S^{3}, \mathcal{C}_{3}\right) \rightarrow\left(S^{3}, \mathcal{C}_{3}\right)$. Next, let us take $(y, z, x) \in S_{\alpha}(r) \cap \operatorname{int}\left(C_{\Delta}(\mathbb{Z})\right)$. As a consequence of Lemma 2.4, $\overline{(y, z, x)}$ and $-\left(i_{\gamma}\right)_{*}(\overline{(y, z, x)})=\overline{(z, y, x)}$ have the same entropy. In fact, the inverse

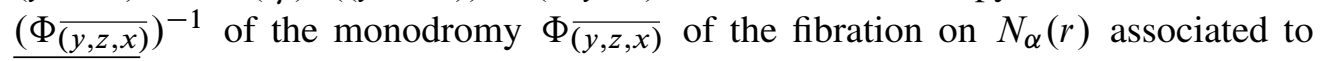
$\overline{(y, z, x)}$ is conjugate to the monodromy $\Phi \overline{(z, y, x)}$ of the fibration on $N_{\beta}(r)$ associated to $\overline{(z, y, x)}$. Putting all things together, we see that for $(x, y, z) \in S_{\beta}(r) \cap \operatorname{int}\left(C_{\Delta_{2}}(\mathbb{Z})\right)$, the two fibered classes $\overline{(x, y, z)} \in \operatorname{int}\left(C_{\Omega_{A, \Delta_{2}}}(\mathbb{Z})\right)$ and $\overline{(z, y, x)} \in \operatorname{int}\left(C_{\Omega_{A, \Delta}}(\mathbb{Z})\right)$ have the same entropy. This completes the proof.

Because of the lemma above, we denote by $\Omega_{A}=\Omega_{A, r}$ (resp. $\Omega_{S}=\Omega_{S, r}$ ), any $A$-face (resp. $S$-face) of $N(r)$. The first letter ' $A$ ' (resp. ' $S$ ') represents 'asymmetry' (resp. 'symmetry'); cf Remark 3.4.

Lemma 2.23 Let $a \in S_{\beta}\left(\frac{p}{q}\right) \subset H_{2}(N, \partial N ; \mathbb{Z})$ be a primitive fibered class, and let $\Omega$ be the fibered face of $N\left(\frac{p}{q}\right)$ such that $\bar{a} \in \operatorname{int}\left(C_{\Omega}\right) \subset H_{2}\left(N_{\beta}(r), \partial N_{\beta}(r)\right)$. If $\Omega$ is an $S$-face (resp. A-face), then $\mathcal{F}_{a}$ has the property that each boundary component on $T_{\beta}$ has $p+2 q$ prongs (resp. $|q|$ prongs). The inequality $\lambda_{p / q}(\bar{a}) \leq \lambda(a)$ holds, and the equality is achieved if $p+2 q \neq 1$ (resp. if $|q| \neq 1$ ).

Note that $p+2 q \geq 1$ when $\Omega$ is an $S$-face (cf Remark 2.3).

Proof Let $\mathcal{S}=\mathcal{S}_{a}$ be the suspended stable foliation constructed from $\mathcal{F}_{a} \times I \subset F_{a} \times I$ by gluing $\mathcal{F}_{a} \times\{1\}$ to $\mathcal{F}_{a} \times\{0\}$ using $\Phi_{a}$. It is known that such a foliation $\mathcal{S}$ depends 
only on the fibered face, that is $\mathcal{S}_{a}$ is isotopic to $\mathcal{S}_{a^{\prime}}$ if $a$ and $a^{\prime}$ are primitive fibered classes in the cone over the same fibered face; see McMullen [26, Corollary 3.2]. When $a \in S_{\beta}(r)$, the number of prongs on each boundary component of $T_{\beta}$ is determined by how $\mathcal{S}$ intersects with the simple closed curve representing the slope $r$ on $T_{\beta}$. Thus such a number depends only on the slope $r$ and the fibered face.

Given a fibered face $\Omega$ of $N\left(\frac{p}{q}\right)$, it is enough to take one primitive fibered class $a \in S_{\beta}\left(\frac{p}{q}\right)$ such that $\bar{a} \in \operatorname{int}\left(C_{\Omega}\right)$. Then one can apply Lemma 2.2 to know the desired number of prongs on each boundary component. Let us compute the desired number when $r=p / q \in(-2,0)$. (The rest of the cases can be proved similarly.) In this case, every face of $N(r)$ is an $A$-face. One sees that $\mathfrak{a}_{r} \in \operatorname{int}\left(C_{\Delta}\right) \cap S_{\beta}(r)$ and $\overline{\mathfrak{a}}_{r} \in \operatorname{int}\left(C_{\Omega_{A}}\right)$. By Lemma 2.2, the desired number equals $|q|$.

The second half of the claim on the inequality between $\lambda_{p / q}(\bar{a})$ and $\lambda(a)$ is clear. The equality holds if $\mathcal{F}_{a}$ has the property such that any boundary component on $T_{\beta}$ has no 1 prong.

Proposition 2.24 For $r \in \mathcal{H} y p$, there exists a fibered face $\Omega$ of $N(r)$ which enjoys (*) in Theorem 1.11.

Proof We use Lemma 2.23. Let $p \in \mathbb{N}$ and $q \in \mathbb{Z}$ be coprime such that $\frac{p}{q} \in \mathcal{H} y p$. There exists no pair $(p, q)$ such that $|q|=1$ and $p+2 q=1$.

Suppose that $|q| \neq 1$ and $p+2 q \neq 1$. Then each fibered face of $N(r)$ enjoys $(*)$ in Theorem 1.11. Suppose that $|q| \neq 1$ and $p+2 q=1$ (resp. Suppose that $|q|=1$ and $p+2 q \neq 1$ ). Then only $A$-faces (resp. only $S$-faces) of $N(r)$ fulfill (*).

Lemma 2.25 For $r=\frac{p}{q} \in \mathcal{H} y p$, let $\Omega$ be a fibered face of $N(r)$ enjoying (*) in Theorem 1.11. We take $a \in S_{\beta}(r) \cap \operatorname{Int} C$ such that $\|a\|=1$.

(1) If $\Omega=\Omega_{S}$ and $\bar{a} \in \operatorname{int}\left(C_{\Omega_{S}}\right) \subset H_{2}\left(N_{\beta}(r), \partial N_{\beta}(r)\right)$, then

$$
\operatorname{Ent}_{r}(\bar{a})=\left(1-\frac{1}{p+q}\right) \log \lambda(\sigma(a)) .
$$

(2) If $\Omega=\Omega_{A}$ and $\bar{a} \in \operatorname{int}\left(C_{\Omega_{A}}\right) \subset H_{2}\left(N_{\beta}(r), \partial N_{\beta}(r)\right)$, then

$$
\begin{aligned}
& \operatorname{Ent}_{r}(\bar{a})=\left(1-\left|\frac{y}{q}\right|\right) \log \lambda(\sigma(a)) \text { when } \sigma(a)=(x, y, z) \in \operatorname{int}(\Delta) \cap S_{\beta}(r), \\
& \operatorname{Ent}_{r}(\bar{a})=\left(1-\left|\frac{x}{q}\right|\right) \log \lambda(\sigma(a)) \text { when } \sigma(a)=(x, y, z) \in \operatorname{int}(\Delta) \cap S_{\alpha}(r) .
\end{aligned}
$$


Proof (1) We have $\sigma(a) \in \operatorname{int}(\Delta) \cap S_{\gamma}(r)$ since $\bar{a} \in \operatorname{int}\left(C_{\Omega_{S}}\right)$; see Lemma 2.21. The Thurston norms of both classes $\bar{a} \in H_{2}\left(N_{\beta}(r), \partial N_{\beta}(r)\right)$ and $\overline{\sigma(a)} \in H_{2}\left(N_{\gamma}(r), \partial N_{\gamma}(r)\right)$ are equal, and hence $\|\bar{a}\|_{r}=\|\overline{\sigma(a)}\|_{r}=1-1 /(p+q)$ by Lemma 2.15. On the other hand, the condition $(*)$ in Theorem 1.11 ensures that $\lambda_{r}(\bar{a})$ is equal to $\lambda(a)$. We have the equality $\lambda(a)=\lambda(\sigma(a))$, since the monodromies $\Phi_{a}$ and $\Phi_{\sigma(a)}$ are conjugate. Thus

$$
\operatorname{Ent}_{r}(\bar{a})=\|\bar{a}\|_{r} \log \lambda_{r}(\bar{a})=\left(1-\frac{1}{p+q}\right) \log \lambda(\sigma(a)) .
$$

(2) By using Lemma 2.16, one can prove the claim similarly.

Theorem 2.26 Let $p \in \mathbb{N}$ and $q \in \mathbb{Z}$ be coprime such that $\frac{p}{q} \in \mathcal{H} y p$.

(1) Suppose $\frac{p}{q} \in(-\infty,-2)$ and $p+2 q \neq 1$. Then $\left(N\left(\frac{p}{q}\right), \Omega_{S}\right) \underset{\text { ent }}{\sim}\left(N\left(\frac{2 q+p}{-q}\right), \Omega_{S}\right)$.

(2) Suppose $\frac{p}{q} \in(-\infty,-1)$ and $|q| \neq 1$. Then $\left(N\left(\frac{p}{q}\right), \Omega_{A}\right) \underset{\text { ent }}{\sim}\left(N\left(\frac{-2 q-p}{q}\right), \Omega_{A}\right)$.

(3) Suppose $\frac{p}{q} \in(-\infty,-1), p+2 q \neq 1$ and $|q| \neq 1$. Then $N\left(\frac{p}{q}\right) \underset{\text { ent }}{\sim} N\left(\frac{-2 q-p}{q}\right)$.

Proof For $r=\frac{p}{q} \in \mathcal{H} y p \cap(-\infty,-1)$, set $r^{\prime}=-2-r$. Recall that

$$
f: H_{2}(N(r), \partial N(r) ; \mathbb{Z}) \rightarrow H_{2}\left(N\left(r^{\prime}\right), \partial N\left(r^{\prime}\right) ; \mathbb{Z}\right)
$$

is the Thurston norm preserving isomorphism as in the proof of Lemma 2.19 and Proposition 2.20. Then $f$ maps $A$-faces (resp. $S$-faces) of $N(r)$ to $A$-faces (resp. $S$-faces) of $N\left(r^{\prime}\right)$.

Let $b_{0}, \tilde{b}_{0}, \ldots, b_{3}, \tilde{b}_{3} \in \operatorname{int}(\Delta)$ be as in Remark 2.8 .

(1) Let $\Omega_{S, r}$ (resp. $\Omega_{S, r^{\prime}}$ ) be the $S$-face of $N(r)$ (resp. $\left.N\left(r^{\prime}\right)\right)$ coming from $\Delta_{1}$ of $N$. Observe that

$$
f\left(\operatorname{int}\left(C_{\Omega_{S, r}}(\mathbb{Z})\right)\right)=\operatorname{int}\left(C_{\Omega_{S, r^{\prime}}}(\mathbb{Z})\right) .
$$

It suffices to prove that for each $a \in \operatorname{int}\left(\Delta_{1}\right) \cap S_{\beta}(r)$, the two classes

$$
\begin{gathered}
\bar{a} \in \operatorname{int}\left(C_{\Omega_{S, r}}\right) \subset H_{2}\left(N_{\beta}(r), \partial N_{\beta}(r)\right), \\
f(\bar{a}) \in \operatorname{int}\left(C_{\Omega_{S, r^{\prime}}}\right) \subset H_{2}\left(N_{\beta}\left(r^{\prime}\right), \partial N_{\beta}\left(r^{\prime}\right)\right),
\end{gathered}
$$

have the same entropy. To do this, consider the sets $\operatorname{int}(\Delta) \cap S_{\gamma}(r)$ and $\operatorname{int}(\Delta) \cap S_{\gamma}\left(r^{\prime}\right)$ which are the images of $\operatorname{int}\left(\Delta_{1}\right) \cap S_{\beta}(r)$ and $\operatorname{int}\left(\Delta_{1}\right) \cap S_{\beta}\left(r^{\prime}\right)$ under $\sigma$. If we write $b_{0}=\sigma(a) \in \operatorname{int}(\Delta) \cap S_{\gamma}(r)$, then we have $\tilde{b}_{2}=\sigma\left(a^{\prime}\right) \in \operatorname{int}(\Delta) \cap S_{\gamma}\left(r^{\prime}\right)$, 
where $a^{\prime}=\iota_{\beta}(f(\bar{a})) \in \operatorname{int}\left(\Delta_{1}\right) \cap S_{\beta}\left(r^{\prime}\right)$ (see Figure 7(right)). As a consequence of Corollary 2.7 and Lemma 2.23, it follows that

$$
\operatorname{ent}_{r}\left(\bar{b}_{0}\right)=\operatorname{ent}_{r^{\prime}}\left(\overline{\tilde{b}}_{2}\right)
$$

ie, $\bar{b}_{0} \in H_{2}\left(N_{\gamma}(r), \partial N_{\gamma}(r)\right)$ and $\overline{\widetilde{b}}_{2} \in H_{2}\left(N_{\gamma}\left(r^{\prime}\right), \partial N_{\gamma}\left(r^{\prime}\right)\right)$ have the same entropy. Since we have

$$
\operatorname{ent}_{r}\left(\bar{b}_{0}\right)=\operatorname{ent}_{r}(\bar{a}) \text { and } \operatorname{ent}_{r^{\prime}}\left(\overline{\tilde{b}}_{2}\right)=\operatorname{ent}_{r^{\prime}}(f(\bar{a})),
$$

we conclude that $\operatorname{ent}_{r}(\bar{a})=\operatorname{ent}_{r^{\prime}}(f(\bar{a}))$. This completes the proof.

(2) Let $\Omega_{A, r}$ be the $A$-face of $N(r)$ coming from $\Delta$ of $N$, and let $\Omega_{A, r^{\prime}}$ be the $A$-face of $N(r)$ coming from $\Delta_{2}^{\prime}$ of $N$. One sees that

$$
f\left(\operatorname{int}\left(C_{\Omega_{A, r}}(\mathbb{Z})\right)\right)=\operatorname{int}\left(C_{\Omega_{A, r^{\prime}}}(\mathbb{Z})\right) .
$$

It is enough to prove that for each $a \in \operatorname{int}(\Delta) \cap S_{\beta}(r)$, the two classes

$$
\begin{gathered}
\bar{a} \in \operatorname{int}\left(C_{\Omega_{A, r}}\right) \subset H_{2}\left(N_{\beta}(r), \partial N_{\beta}(r)\right), \\
f(\bar{a}) \in \operatorname{int}\left(C_{\Omega_{A, r^{\prime}}}\right) \subset H_{2}\left(N_{\beta}\left(r^{\prime}\right), \partial N_{\beta}\left(r^{\prime}\right)\right),
\end{gathered}
$$

have the same entropy. Now, we consider the sets $\operatorname{int}(\Delta) \cap S_{\beta}(r)$ and $\operatorname{int}(\Delta) \cap S_{\alpha}\left(r^{\prime}\right)$ which are the images of $\operatorname{int}(\Delta) \cap S_{\beta}(r)$ and $\operatorname{int}\left(\Delta_{2}^{\prime}\right) \cap S_{\beta}\left(r^{\prime}\right)$ under $\sigma$. If one writes $b_{0}=\sigma(a) \in \operatorname{int}(\Delta) \cap S_{\beta}(r)$, then one can write $\tilde{b}_{0}=\sigma\left(a^{\prime}\right) \in \operatorname{int}(\Delta) \cap S_{\alpha}\left(r^{\prime}\right)$, where $a^{\prime}=\iota_{\beta}(f(\bar{a})) \in \operatorname{int}\left(\Delta_{2}^{\prime}\right) \cap S_{\beta}\left(r^{\prime}\right)$. As a consequence of Corollary 2.7 and Lemma 2.23, it follows that

$$
\operatorname{ent}_{r}\left(\bar{b}_{0}\right)=\operatorname{ent}_{r^{\prime}}\left(\overline{\widetilde{b}}_{0}\right)
$$

ie, $\bar{b}_{0} \in H_{2}\left(N_{\beta}(r), \partial N_{\beta}(r)\right)$ and $\overline{\widetilde{b}}_{0} \in H_{2}\left(N_{\alpha}\left(r^{\prime}\right), \partial N_{\alpha}\left(r^{\prime}\right)\right)$ have the same entropy.

Since ent ${ }_{r}\left(\bar{b}_{0}\right)=\operatorname{ent}_{r}(\bar{a})$ and $\operatorname{ent}_{r^{\prime}}\left(\overline{\tilde{b}}_{0}\right)=\operatorname{ent}_{r^{\prime}}(f(\bar{a}))$, the map $f$ preserves the entropy, ie, ent $r(\bar{a})=\operatorname{ent}_{r^{\prime}}(f(\bar{a}))$. This completes the proof.

(3) The proof of (3) is similar to that of (1) or (2).

Let us check the entropy equivalence on some pairs which we promised to prove in Section 1.6. Theorem 2.26 tells us that

$$
\left(N(-4), \Omega_{S}\right) \underset{\mathrm{ent}}{\sim}\left(N(2), \Omega_{S}\right), \quad N\left(\frac{3}{-2}\right) \underset{\mathrm{ent}}{\sim} N\left(\frac{1}{-2}\right) .
$$

Since $N(-4) \simeq N\left(\frac{3}{-2}\right)$, we see that

$$
\left(N(2), \Omega_{S}\right) \underset{\mathrm{ent}}{\sim}\left(N\left(\frac{3}{-2}\right), \Omega_{A}\right) \underset{\mathrm{ent}}{\sim}\left(N\left(\frac{1}{-2}\right), \Omega_{A}\right) .
$$




\section{Proofs of main results}

\subsection{Proof of Theorem 1.11 (Theorem 3.1)}

In this subsection, we shall prove the next theorem which is equivalent to Theorem 1.11 (see Lemma 2.23 or proof of Proposition 2.24).

Theorem 3.1 Let $p \in \mathbb{N}$ and $q \in \mathbb{Z}$ be coprime such that $\frac{p}{q} \in \mathcal{H} y p$.

(1) Suppose that $\frac{p}{q} \in(-\infty,-2) \cup(0, \infty)$ and $p+2 q \neq 1$. Then

(i) $\min \operatorname{Ent}\left(N(1), \Omega_{S}\right)=2 \log \delta\left(D_{4}\right) \approx 1.6628$,

(ii) $\min \operatorname{Ent}\left(N\left(\frac{p}{q}\right), \Omega_{S}\right)=2 \log \left(\frac{3+\sqrt{5}}{2}\right) \approx 1.9248$ for $\frac{p}{q}=-4,2$,

(iii) $\min \operatorname{Ent}\left(N\left(\frac{p}{q}\right), \Omega_{S}\right)>1.97475$ if $\frac{p}{q} \neq-4,1,2$.

(2) Suppose that $|q| \neq 1$. Then

(i) $\min \operatorname{Ent}\left(N\left(\frac{p}{q}\right), \Omega_{A}\right)=2 \log \left(\frac{3+\sqrt{5}}{2}\right) \approx 1.9248$ for $\frac{p}{q}=\frac{3}{-2}, \frac{1}{-2}$,

(ii) $\min \operatorname{Ent}\left(N\left(\frac{p}{q}\right), \Omega_{A}\right)>1.97475$ if $\frac{p}{q} \neq \frac{3}{-2}, \frac{1}{-2}$.

We start by the computation of $\min \operatorname{Ent}\left(N\left(\frac{p}{q}\right), \Omega_{S}\right)$ and $\min \operatorname{Ent}\left(N\left(\frac{p}{q}\right), \Omega_{A}\right)$.

Lemma 3.2 Let $\frac{p}{q} \in(-\infty,-2) \cup(0, \infty)$. Then $\min \operatorname{Ent}\left(N\left(\frac{p}{q}\right), \Omega_{S}\right)=\left(1-\frac{1}{p+q}\right) \log \lambda_{[p /(2 p+2 q), p /(2 p+2 q)]} \quad$ if $p+2 q \neq 1$.

Proof By Lemma 2.22, we have the equalities $\min \operatorname{Ent}\left(N\left(\frac{p}{q}\right), \Omega_{S}\right)$

$$
\begin{aligned}
& =\min \left\{\|\overline{[x, y]}\|_{p / q} \log \lambda_{p / q}(\overline{[x, y]}) \mid[x, y] \in \operatorname{int}(\Delta) \cap S_{\gamma}\left(\frac{p}{q}\right)\right\} \\
& =\min \left\{\left(1-\frac{1}{p+q}\right) \log \lambda_{p / q}(\overline{[x, y]}) \mid[x, y] \in \operatorname{int}(\Delta) \cap S_{\gamma}\left(\frac{p}{q}\right)\right\} \\
& =\min \left\{\left(1-\frac{1}{p+q}\right) \log \lambda_{[x, y]} \mid[x, y] \in \operatorname{int}(\Delta) \cap S_{\gamma}\left(\frac{p}{q}\right)\right\} .
\end{aligned}
$$

The first equality comes from Lemma 2.21(2). The second equality and the third one follow from Lemma 2.15 and Lemma 2.23 respectively. Lemmas 2.9(3) and 2.12(3) imply that the minimum is achieved by the center $\left[\frac{p}{2 p+2 q}, \frac{p}{2 p+2 q}\right] \in \operatorname{int}(\Delta) \cap S_{\gamma}\left(\frac{p}{q}\right)$. This completes the proof. 
Lemma 3.3 Let $\frac{p}{q} \in(-\infty, \infty)$. Then

$$
\begin{aligned}
\min \operatorname{Ent}\left(N\left(\frac{p}{q}\right), \Omega_{A}\right) & =\min \left\{\left(1-\left|\frac{y}{q}\right|\right) \log \lambda_{[x, y]} \mid[x, y] \in \operatorname{int}(\Delta) \cap S_{\beta}\left(\frac{p}{q}\right)\right\} \\
& =\min \left\{\left(1-\left|\frac{x}{q}\right|\right) \log \lambda_{[x, y]} \mid[x, y] \in \operatorname{int}(\Delta) \cap S_{\alpha}\left(\frac{p}{q}\right)\right\} .
\end{aligned}
$$

Proof The claim follows from Lemmas 2.21(1), (3) and 2.22.

Remark 3.4 If an $S$-face enjoys (*) in Theorem 1.11, then we are able to compute $\min \operatorname{Ent}\left(N(r), \Omega_{S}\right)$ explicitly from Lemma 3.2. This is because ent: $\operatorname{int}(\Delta) \rightarrow \mathbb{R}$ on $\operatorname{int}(\Delta) \cap S_{\gamma}\left(\frac{p}{q}\right)$ has a symmetry with respect to the center. There exists no symmetry of ent: $\operatorname{int}(\Delta) \rightarrow \mathbb{R}$ on $\operatorname{int}(\Delta) \cap S_{\beta}\left(\frac{p}{q}\right)$ (hence on $\left.\operatorname{int}(\Delta) \cap S_{\alpha}\left(\frac{p}{q}\right)\right)$ in general. Later we shall compute $\min \operatorname{Ent}\left(N(r), \Omega_{A}\right)$ for some manifolds having a symmetry themselves (see Lemma 3.6 and Proposition 3.26), but these cases are rare.

We prove the following monotonicity of $\min \operatorname{Ent}\left(\cdot, \Omega_{A}\right)$.

Lemma 3.5 Let $p, p^{\prime} \in \mathbb{N}$ and $q \in \mathbb{Z}$ such that $(p, q)$ and $\left(p^{\prime}, q\right)$ are coprime pairs. If $|q| \neq 1$ and $\left|1+\frac{p^{\prime}}{q}\right|>\left|1+\frac{p}{q}\right|$, then $\min \operatorname{Ent}\left(N\left(\frac{p^{\prime}}{q}\right), \Omega_{A}\right)>\min \operatorname{Ent}\left(N\left(\frac{p}{q}\right), \Omega_{A}\right)$.

Proof We use Lemma 3.3. Put $r^{\prime}=\frac{p^{\prime}}{q}$ and $r=\frac{p}{q}$. The sets $\Delta \cap S_{\beta}\left(r^{\prime}\right)$ and $\Delta \cap S_{\beta}(r)$ lie on the lines $y=\left(\frac{-2}{1+r^{\prime}}\right) x+\frac{1}{1+r^{\prime}}$ and $y=\left(\frac{-2}{1+r}\right) x+\frac{1}{1+r}$ respectively. (These lines go through $\left[\frac{1}{2}, 0\right] \in \partial \Delta$.) One has the inequality $\left|\frac{-2}{1+r^{\prime}}\right|<\left|\frac{-2}{1+r}\right|$ between the slopes. Thus for any $a^{\prime}=\left[x^{\prime}, y^{\prime}\right] \in \operatorname{int}(\Delta) \cap S_{\beta}\left(r^{\prime}\right)$, there exists a unique point $a=\left[x, y^{\prime}\right] \in \operatorname{int}(\Delta) \cap S_{\beta}(r)$ with the same second coordinate $y^{\prime}$. Since $\left|\frac{1}{2}-x\right|<\left|\frac{1}{2}-x^{\prime}\right|$, one sees that $\lambda\left[x, y^{\prime}\right]<\lambda_{\left[x^{\prime}, y^{\prime}\right]}$ (cf Lemma 2.9(2)). The condition $|q| \neq 1$ says that $A$ faces for both $N\left(\frac{p^{\prime}}{q}\right)$ and $N\left(\frac{p}{q}\right)$ enjoy (*) in Theorem 1.11. Hence by Lemma 2.25(2),

$$
\text { Ent }_{r^{\prime}}\left(\bar{a}^{\prime}\right)=\left(1-\left|\frac{y^{\prime}}{q}\right|\right) \lambda_{\left[x^{\prime}, y^{\prime}\right]}>\left(1-\left|\frac{y^{\prime}}{q}\right|\right) \lambda_{\left[x, y^{\prime}\right]}=\operatorname{Ent}_{r}(\bar{a}) .
$$

Since this holds for any $a^{\prime} \in \operatorname{int}(\Delta) \cap S_{\beta}\left(r^{\prime}\right)$, the proof is done.

Lemma 3.6 Suppose that $|q|=2$. Then:

(1) $\min \operatorname{Ent}\left(N\left(\frac{p}{q}\right), \Omega_{A}\right)=2 \log \left(\frac{3+\sqrt{5}}{2}\right)$ if $\frac{p}{q}=\frac{3}{-2}, \frac{1}{-2}$.

(2) $\min \operatorname{Ent}\left(N\left(\frac{p}{q}\right), \Omega_{A}\right)=4 \log \lambda_{(4,2,1)} \approx 2.5318$ if $\frac{p}{q}=\frac{5}{-2}, \frac{1}{2}$.

(3) $\min \operatorname{Ent}\left(N\left(\frac{p}{q}\right), \Omega_{A}\right)>4 \log \lambda_{(4,2,1)}$ otherwise. 
Proof For the proof of (1), see [19, Proposition 4.13]. In fact in this case, the center of each $A$-face $\Omega_{A}$ reaches $\min \operatorname{Ent}\left(N\left(\frac{p}{q}\right), \Omega_{A}\right)$.

Let us turn to the proof of (2). By Theorem 2.26, $N\left(\frac{5}{-2}\right)$ and $N\left(\frac{1}{2}\right)$ are entropy equivalent. Put $r_{0}=5 /(-2)$. We consider the $A$-face $\Omega_{A}$ (on $\partial B_{r_{0}}(2)$ ) whose endpoints are $\overline{(3,2,2)}, \overline{(1,0,-1)} \in H_{2}\left(N\left(r_{0}\right), \partial N\left(r_{0}\right)\right)$. We now prove that ent $\left.r_{0}\right|_{\Omega_{A}}$ has a minimum at the center of $\Omega_{A}$. The ray from the origin and through $\overline{(4,2,1)} \in$ $\operatorname{int}\left(C_{\Omega_{A}}\right)$ passes through the center of $\Omega_{A}$. (In other words, the ray from the origin, through $(2,-1)$ in the $\left(\overline{\mathfrak{a}}_{r_{0}}, \overline{\mathfrak{b}}_{r_{0}}\right)$ coordinates, passes through the center of $\Omega_{A}$; see Figure 9.) For $k>\ell$, let

$$
p_{ \pm}(k, \ell)=(4,2,1) k \pm(2,2,3) \ell=(4 k \pm 2 \ell, 2 k \pm 2 \ell, k \pm 3 \ell) .
$$

Observe that $p_{ \pm}(k, \ell)$ are elements of $\operatorname{int}\left(C_{\Delta}\right) \cap S_{\beta}\left(r_{0}\right)$, and $\overline{p_{ \pm}(k, \ell)} \in \operatorname{int}\left(C_{\Omega_{A}}\right)$ have the same Thurston norm. To show that the center of $\Omega_{A}$ achieves the minimum of ent $r_{0} \mid \Omega_{A}$, it suffices to prove that $\overline{p_{+}(k, \ell)}$ and $\overline{p_{-}(k, \ell)}$ have the same entropy for each $k, \ell$ such that $k>\ell$. To do this, we show that $p_{+}(k, \ell)$ and $p_{-}(k, \ell)$ have the same dilatation (since in this case, $\lambda(a)=\lambda_{r_{0}}(\bar{a})$ for $a \in S_{\beta}\left(r_{0}\right)$ such that $\left.\bar{a} \in \operatorname{int}\left(C_{\Omega_{A}}\right)\right)$. The dilatation $\lambda\left(p_{+}(k, \ell)\right)\left(\right.$ resp. $\left.\lambda\left(p_{-}(k, \ell)\right)\right)$ is the largest real root of the polynomial $f_{(4 k+2 \ell, 2 k+2 \ell, k+3 \ell)}(t)$ (resp. $f_{(4 k-2 \ell, 2 k-2 \ell, k-3 \ell)}(t)$ ), that is

$$
\begin{array}{r}
\quad-t^{-\ell}\left(1+t^{k+\ell}\right)\left(t^{k}+t^{3 k}-t^{\ell}-t^{2 k+\ell}-t^{4 k+\ell}+t^{k+2 \ell}+t^{3 k+2 \ell}\right), \\
\left(\text { resp. }-t^{-2 \ell}\left(t^{k}+t^{\ell}\right)\left(t^{k}+t^{3 k}-t^{\ell}-t^{2 k+\ell}-t^{4 k+\ell}+t^{k+2 \ell}+t^{3 k+2 \ell}\right)\right) .
\end{array}
$$

Since each of polynomials $-t^{-2 \ell}\left(t^{k}+t^{\ell}\right)$ and $-t^{-\ell}\left(1+t^{k+\ell}\right)$ have no real roots greater than 1 , the proof of (2) is done.

The claim (2) together with Lemma 3.5 leads to (3).

Lemma 3.7 Suppose that $|q|=3$. Then $\min \operatorname{Ent}\left(N\left(\frac{p}{q}\right), \Omega_{A}\right)>2.0918$.

Proof By Lemma 3.5 and Theorem 2.26,

$$
\min \operatorname{Ent}\left(N\left(\frac{p}{q}\right), \Omega_{A}\right)>\min \operatorname{Ent}\left(N\left(\frac{2}{-3}\right), \Omega_{A}\right)=\min \operatorname{Ent}\left(N\left(\frac{4}{-3}\right), \Omega_{A}\right)
$$

if $|q|=3$ and $\frac{p}{q} \neq \frac{2}{-3}, \frac{4}{-3}$. Thus it suffices to prove that $\min \operatorname{Ent}\left(N\left(\frac{2}{-3}\right), \Omega_{A}\right)>2.0918$. We consider the $A$-face $\Omega_{A}$ (on $\left.\partial B_{2 /-3}(2)\right)$ for $N\left(\frac{2}{-3}\right)$ whose endpoints are $\overline{(1,3,1)}$ and $\overline{(1,0,-1)} \in H_{2}\left(N\left(\frac{2}{-3}\right), \partial N\left(\frac{2}{-3}\right)\right)$. Take fibered classes

$a_{1}=(201,312,7), \quad a_{2}=(201,309,5), \quad a_{3}=(201,306,3) \in \operatorname{int}\left(C_{\Delta}\right) \cap S_{\beta}\left(\frac{2}{-3}\right)$. 
Then $\bar{a}_{1}, \bar{a}_{2}$ and $\bar{a}_{3}$ are elements of $\operatorname{int}\left(C_{\Omega_{A}}\right)$. One can check that the Thurston norms of $\bar{a}_{1}, \bar{a}_{2}$ and $\bar{a}_{3}$ are the same. Note that $\lambda(a)=\lambda_{2 /(-3)}(\bar{a})$ for $a \in S_{\beta}\left(\frac{2}{-3}\right)$ such that $\bar{a} \in \operatorname{int}\left(C_{\Omega_{A}}\right)$. One sees that:

$$
\begin{aligned}
\lambda_{(201,312,7)}=1.00542189 \ldots & >\lambda_{(201,309,5)}=1.00542166 \ldots \\
<\lambda_{(201,306,3)} & =1.00542185 \ldots
\end{aligned}
$$

The fibered class $a_{1}$ equals $\left(\frac{201}{506}, \frac{312}{506}, \frac{7}{506}\right) \in \operatorname{int}(\Delta)$ projectively. Thus we have $\min \operatorname{Ent}\left(N\left(\frac{2}{-3}\right), \Omega_{A}\right)$ is achieved by a unique point $[x, y] \in \operatorname{int}(\Delta) \cap S_{\beta}\left(\frac{2}{3}\right)$ such that $0<y<312 / 506$. This together with Lemma 3.3 implies that

$$
\begin{aligned}
\min \operatorname{Ent}(N & \left.\left(\frac{2}{-3}\right), \Omega_{A}\right) \\
& =\min \left\{\left(1-\left|\frac{y}{-3}\right|\right) \log \lambda_{[x, y]} \mid[x, y] \in \operatorname{int}(\Delta) \cap S_{\beta}\left(\frac{2}{-3}\right), 0<y<\frac{312}{506}\right\} \\
& >\left(1-\frac{312}{3 \times 506}\right) \log \lambda_{[1 / 2,1 / 2]}>\frac{402}{506} \times 2.633>2.0918 .
\end{aligned}
$$

This completes the proof.

\section{Lemma 3.8}

(1) Let $\frac{p}{q} \in(-\infty,-2) \cup(0, \infty)$ such that $p+2 q \neq 1$. Suppose that $p+q \geq 4$. Then $\min \operatorname{Ent}\left(N\left(\frac{p}{q}\right), \Omega_{S}\right)>1.97475$.

(2) Suppose that $|q| \geq 4$. Then $\min \operatorname{Ent}\left(N\left(\frac{p}{q}\right), \Omega_{A}\right)>1.97475$.

Proof The claim (1) is immediate from

$$
\begin{aligned}
\min \operatorname{Ent}\left(N\left(\frac{p}{q}\right), \Omega_{S}\right) & =\left(1-\frac{1}{p+q}\right) \log \lambda_{[p /(2 p+2 q), p /(2 p+2 q)]} \\
& >\left(1-\frac{1}{4}\right) \times 2.633=1.97475 .
\end{aligned}
$$

Let us turn to the claim (2). By Lemma 3.3,

$$
\min \operatorname{Ent}\left(N\left(\frac{p}{q}\right), \Omega_{A}\right)=\min \left\{\left(1-\left|\frac{y}{q}\right|\right) \log \lambda_{[x, y]} \mid[x, y] \in \operatorname{int}(\Delta) \cap S_{\beta}\left(\frac{p}{q}\right)\right\} .
$$

Since $|q| \geq 4$, one sees that for any $[x, y] \in \operatorname{int}(\Delta) \cap S_{\beta}\left(\frac{p}{q}\right)$,

$$
\left(1-\left|\frac{y}{q}\right|\right) \log \lambda_{[x, y]}>\left(1-\frac{1}{4}\right) \times 2.633=1.97475 .
$$

This completes the proof. 
Proof of Theorem 3.1 We have already proved the claim (2); see Lemmas 3.6, 3.7 and 3.8(2). Let us prove the claim (1). By Lemma 3.8(1), it is enough to consider the case $p+q<4$. For $\frac{p}{q} \in \mathcal{H} y p \cap(-\infty,-2)$ such that $p+2 q \neq 1$, one has by Theorem 2.26,

$$
\min \operatorname{Ent}\left(N\left(\frac{p}{q}\right), \Omega_{S}\right)=\min \operatorname{Ent}\left(N\left(\frac{-2 q-p}{q}\right), \Omega_{S}\right) .
$$

If $\frac{p}{q} \in \mathcal{H} y p \cap(0, \infty)$, then $p+2 q \geq 3$ (hence $p+2 q \neq 1$ ). Thus it suffices to consider the case $\frac{p}{q} \in \mathcal{H} y p \cap(0, \infty)$ such that $p+q<4$. The pairs $(p, q)$ with $p+q<4$ are given by $(p, q)=(1,1),(2,1),(1,2)$. By Lemma 3.2,

$$
\begin{aligned}
& \min \operatorname{Ent}\left(N\left(\frac{1}{1}\right), \Omega_{S}\right)=2 \log \lambda_{(1,1,-2)}=2 \log \delta\left(D_{4}\right) \approx 1.6628, \\
& \min \operatorname{Ent}\left(N\left(\frac{2}{1}\right), \Omega_{S}\right)=2 \log \lambda_{(1,1,-1)}=2 \log \left(\frac{3+\sqrt{5}}{2}\right) \approx 1.9248, \\
& \min \operatorname{Ent}\left(N\left(\frac{1}{2}\right), \Omega_{S}\right)=4 \log \lambda_{(1,1,-4)} \approx 2.9314 .
\end{aligned}
$$

This completes the proof.

\subsection{Proof of Theorem 1.4(1)}

The idea of the proof is as follows. We define a finite set $L_{K} \subset \mathcal{H} y p$ for $K>2$ which consists of irreducible rational numbers $\frac{p}{q} \in \mathcal{H} y p$ with $p \in \mathbb{N}$ such that

- $|q| \leq K$ if $\frac{p}{q} \in(-2,0)$,

- $p+q \leq K$ if $\frac{p}{q} \in(-\infty,-2) \cup(0, \infty)$.

We fix $K_{0}=100000$. First we prove that for a primitive fibered class $a \in H_{2}(N, \partial N)$ such that $\phi_{a} \in \mathcal{M}$, the normalized entropy of $\hat{\phi}_{a}$ is greater than 2.5803 if all the slopes $b_{\alpha}(a), b_{\beta}(a), b_{\gamma}(a)$ of $a$ enjoy $b_{\alpha}(a), b_{\beta}(a), b_{\gamma}(a) \in \mathcal{H} y p \backslash L_{K_{0}}$. Next we prove the following for any $\epsilon>0$ : for all but finitely many primitive fibered classes $a \in H_{2}(N, \partial N)$ satisfying $\phi_{a} \in \mathcal{M}$, if one of the boundary slopes of $a$ is an element of $L_{K_{0}}$, then the normalized entropy of $\widehat{\phi}_{a}$ is greater than $2 \log ((3+\sqrt{5}) / 2)-\epsilon$. These together with Theorem 1.3 lead to Theorem 1.4(1).

Lemma 3.9 Let $a=(x, y, z)$ be a primitive fibered class of $H_{2}(N, \partial N)$ such that $\phi_{a} \in \mathcal{M}$. Then $\phi_{\sigma(a)} \in \mathcal{M}$ for $\sigma(a) \in \operatorname{int}\left(C_{\Delta}\right)$, and

$$
\operatorname{Ent}\left(\hat{\phi}_{a}\right)=\operatorname{Ent}\left(\hat{\phi}_{\sigma(a)}\right)=\left(1-\left|\frac{x^{\prime}}{q_{\alpha}(\sigma(a))}\right|-\left|\frac{y^{\prime}}{q_{\beta}(\sigma(a))}\right|-\left|\frac{z^{\prime}}{q_{\gamma}(\sigma(a))}\right|\right) \log \lambda\left(a^{\prime}\right),
$$

where $a^{\prime}=\left(x^{\prime}, y^{\prime}, z^{\prime}\right)$ is the rational class of $\operatorname{int}(\Delta)$ that is projectively equal to $\sigma(a)$. 
Proof Clearly $\phi_{a} \in \mathcal{M}$ implies $\phi_{\sigma(a)} \in \mathcal{M}$ and $\operatorname{Ent}\left(\hat{\phi}_{a}\right)=\operatorname{Ent}\left(\hat{\phi}_{\sigma(a)}\right)$. The dilatation $\lambda\left(\hat{\phi}_{\sigma(a)}\right)$ equals $\lambda(\sigma(a))\left(=\lambda\left(\phi_{\sigma(a)}\right)\right)$ since $\phi_{\sigma(a)} \in \mathcal{M}$. If we set $\sigma(a)=(x, y, z)$, then

$$
\sharp\left(\partial F_{\sigma(a)}\right)=\left|\frac{x}{q_{\alpha}(\sigma(a))}\right|+\left|\frac{y}{q_{\beta}(\sigma(a))}\right|+\left|\frac{z}{q_{\gamma}(\sigma(a))}\right| .
$$

By definition of the normalized entropy,

$$
\operatorname{Ent}\left(\hat{\phi}_{\sigma(a)}\right)=\left(\|\sigma(a)\|-\left|\frac{x}{q_{\alpha}(\sigma(a))}\right|-\left|\frac{y}{q_{\beta}(\sigma(a))}\right|-\left|\frac{z}{q_{\gamma}(\sigma(a))}\right|\right) \log \lambda(\sigma(a)) .
$$

On the other hand we have $x^{\prime}=x /\|\sigma(a)\|, y^{\prime}=y /\|\sigma(a)\|, z^{\prime}=z /\|\sigma(a)\|$ and $\log \lambda\left(a^{\prime}\right)=\|\sigma(a)\| \log \lambda(\sigma(a))$ since $a^{\prime}$ is projectively equal to $\sigma(a)$. Substituting these equalities for $\left(1-\left|\frac{x^{\prime}}{q_{\alpha}}(\sigma(a))\right|-\left|\frac{y^{\prime}}{q_{\beta}(\sigma(a))}\right|-\left|\frac{z^{\prime}}{q_{\gamma}(\sigma(a))}\right|\right) \log \lambda\left(a^{\prime}\right)$, one finds it is equal to $\operatorname{Ent}\left(\hat{\phi}_{\sigma(a)}\right)$.

Proposition 3.10 Suppose that $b_{\alpha}(a), b_{\beta}(a), b_{\gamma}(a) \in \mathcal{H} y p \backslash L_{K_{0}}$ for a rational class $a=(x, y, z) \in \operatorname{int}(\Delta)$. Then

$$
\left(1-\left|\frac{x}{q_{\alpha}(a)}\right|-\left|\frac{y}{q_{\beta}(a)}\right|-\left|\frac{z}{q_{\gamma}(a)}\right|\right) \log \lambda(a)>2.5803 .
$$

We need the following lemma for the proof of Proposition 3.10.

Lemma 3.11 Let us take $K>K^{\prime}>2$. Let $p \in \mathbb{N}$ and $q \in \mathbb{Z}$ be coprime such that $\frac{p}{q} \in \mathcal{H} y p \backslash L_{K}$. Then the following hold.

(1) If $\left|\frac{p}{q}\right| \leq K^{\prime}$, then $|q| \geq K /\left(1+K^{\prime}\right)$. If $\left|\frac{p}{q}\right|>K^{\prime}$, then $0<y<1 /\left(-1+K^{\prime}\right)$ for any $(x, y, z) \in \operatorname{int}(\Delta) \cap S_{\beta}\left(\frac{p}{q}\right)$.

(2) $\left|\frac{y}{q}\right|<\max \left\{\frac{1+K^{\prime}}{K}, \frac{1}{-1+K^{\prime}}\right\}$ for any $(x, y, z) \in \operatorname{int}(\Delta) \cap S_{\beta}\left(\frac{p}{q}\right)$.

Proof (1) If $\frac{p}{q} \in(-2,0)$, then $\left|\frac{p}{q}\right|<2$. The assumption $\frac{p}{q} \notin L_{K}$ implies that $|q|>K>K /\left(1+K^{\prime}\right)$.

Let us consider the case $\frac{p}{q} \in(-\infty,-2) \cup(0, \infty)$. Suppose that $\left|\frac{p}{q}\right| \leq K^{\prime}$ and $q<0$. Then $p \leq-K^{\prime} q$. One has $p+q>K$ since $\frac{p}{q} \notin L_{K}$. Hence $-q<p-K \leq-K^{\prime} q-K$. One obtains $\left(K^{\prime}-1\right) q \leq-K$. Thus $|q|=-q>K /\left(K^{\prime}-1\right)>K /\left(K^{\prime}+1\right)$.

Suppose that $\left|\frac{p}{q}\right| \leq K^{\prime}$ and $q>0$. In this case $p \leq K^{\prime} q$. Since $p+q>K$, one has $q>K-p \geq K-K^{\prime} q$. Thus $q>K /\left(1+K^{\prime}\right)$. The proof of the first part is done. 
The second part can be proved using Lemma 2.12(2). In fact for $[x, y] \in \operatorname{int}(\Delta) \cap S_{\beta}(r)$ such that $|r|>K^{\prime}>2$, one has $0<y<\frac{-1}{1+r}$ when $r \in(-\infty,-2)$ (resp. $0<y<\frac{1}{1+r}$ when $r \in(0, \infty))$. This leads to the second part.

(2) If $\left|\frac{p}{q}\right| \leq K^{\prime}$, then $\left|\frac{y}{q}\right|<\frac{1}{|q|} \leq\left(1+K^{\prime}\right) / K$ by the first part of (1). If $\left|\frac{p}{q}\right|>K^{\prime}$, then by the second part of (1), we have that $\left|\frac{y}{q}\right|<\frac{1}{|q|} \times 1 /\left(-1+K^{\prime}\right) \leq 1 /\left(-1+K^{\prime}\right)$. These imply the desired inequality.

Similarly, one can prove the following.

Lemma 3.12 Let us take $K>K^{\prime}>2$. Let $p \in \mathbb{N}$ and $q \in \mathbb{Z}$ be coprime such that $\frac{p}{q} \in \mathcal{H} y p \backslash L_{K}$.

(1) If $\left|\frac{p}{q}\right| \leq K^{\prime}$, then $|q| \geq K /\left(1+K^{\prime}\right)$. If $\left|\frac{p}{q}\right|>K^{\prime}$, then $0<x<1 /\left(-1+K^{\prime}\right)$ for any $(x, y, z) \in \operatorname{int}(\Delta) \cap S_{\alpha}\left(\frac{p}{q}\right)$.

(2) $\left|\frac{x}{q}\right|<\max \left\{\frac{1+K^{\prime}}{K}, \frac{1}{-1+K^{\prime}}\right\}$ for any $(x, y, z) \in \operatorname{int}(\Delta) \cap S_{\alpha}\left(\frac{p}{q}\right)$.

Proof of Proposition 3.10 Let $K=K_{0}(=100000)$ and $K^{\prime}=999$. Then since $b_{\gamma}(a)=p_{\gamma}(a) / q_{\gamma}(a) \in \mathcal{H} y p \backslash L_{K_{0}}$ and $b_{\gamma}(a) \in(-\infty,-2) \cup(0, \infty)$, the inequality $p_{\gamma}(a)+q_{\gamma}(a)>K_{0}$ holds. By using the same argument in the proof of Lemma 2.15, we obtain the upper bound on $\left|z / q_{\gamma}(a)\right|$,

$$
\left|\frac{z}{q_{\gamma}(a)}\right|=\left|\frac{1}{p_{\gamma}(a)+q_{\gamma}(a)}\right|<\frac{1}{K_{0}}=\frac{1}{100000} .
$$

By Lemmas 3.11(2) and 3.12(2), we have

$$
\left|\frac{x}{q_{\alpha}(a)}\right|,\left|\frac{y}{q_{\beta}(a)}\right|<\max \left\{\frac{1+K^{\prime}}{K_{0}}, \frac{1}{-1+K^{\prime}}\right\}=\frac{1+K^{\prime}}{K_{0}}=\frac{1}{100} .
$$

Thus we have lower bounds $1-\left|x / q_{\alpha}(a)\right|-\left|y / q_{\beta}(a)\right|-\left|z / q_{\gamma}(a)\right|>1-1 / 50-$ $1 / 100000=0.97999$ and $\log \lambda(a) \geq \log \lambda_{[1 / 2,1 / 2]}>2.633$. These two bounds then give us the desired inequality.

Proposition 3.13 Let $p \in \mathbb{N}$ and $q \in \mathbb{Z}$ be coprime such that $\frac{p}{q} \in \mathcal{H} y p \backslash\{1\}$. Let $\epsilon>0$ be any number.

(1) Suppose that $\frac{p}{q} \in(-\infty,-2) \cup(0, \infty)$ and $p+2 q \neq 1$. Then

$$
\left(1-\left|\frac{x}{q_{\alpha}(a)}\right|-\left|\frac{y}{q_{\beta}(a)}\right|-\left|\frac{z}{q}\right|\right) \log \lambda(a)>\min \operatorname{Ent}\left(N\left(\frac{p}{q}\right), \Omega_{S}\right)-\epsilon
$$

for any rational class $a=(x, y, z) \in \operatorname{int}(\Delta) \cap S_{\gamma}\left(\frac{p}{q}\right)$ but finitely many exceptions. 
(2) Suppose that $|q| \neq 1$. Then

$$
\left(1-\left|\frac{x}{q_{\alpha}(a)}\right|-\left|\frac{y}{q}\right|-\left|\frac{z}{q_{\gamma}(a)}\right|\right) \log \lambda(a)>\min \operatorname{Ent}\left(N\left(\frac{p}{q}\right), \Omega_{A}\right)-\epsilon
$$

for any rational class $a=(x, y, z) \in \operatorname{int}(\Delta) \cap S_{\beta}\left(\frac{p}{q}\right)$ but finitely many exceptions.

The following lemma is needed for the proof of Proposition 3.13.

Lemma 3.14 Let $\epsilon^{\prime}>0$ be any number.

(1) Let $r \in \mathcal{H} y p \backslash\{1\}$ and $r \in(-\infty,-2) \cup(0, \infty)$. Then $\left|x / q_{\alpha}(a)\right|<\epsilon^{\prime}$ and $\left|y / q_{\beta}(a)\right|<\epsilon^{\prime}$ for any rational class $a=(x, y, z) \in \operatorname{int}(\Delta) \cap S_{\gamma}(r)$ but finitely many exceptions.

(2) Let $r \in \mathcal{H} y p \backslash\{1\}$. Then $\left|x / q_{\alpha}(a)\right|<\epsilon^{\prime}$ and $\left|z / q_{\gamma}(a)\right|<\epsilon^{\prime}$ for any rational class $a=(x, y, z) \in \operatorname{int}(\Delta) \cap S_{\beta}(r)$ but finitely many exceptions.

Proof Take $K>K^{\prime}>2$ so that $\max \left\{\left(1+K^{\prime}\right) / K, 1 /\left(-1+K^{\prime}\right)\right\}<\epsilon^{\prime}$. (Note that $1 \in L_{K}$.) We see $r \neq 1$ implies $\operatorname{int}(\Delta) \cap S_{\gamma}(r) \cap S_{\alpha}\left(r^{\prime}\right)$ or $\operatorname{int}(\Delta) \cap S_{\gamma}(r) \cap S_{\beta}\left(r^{\prime}\right)$ is at most a single point for any $r^{\prime}$. This means the set of rational classes $a=(x, y, z) \in$ $\operatorname{int}(\Delta) \cap S_{\gamma}(r)$ such that $b_{\alpha}(a) \in L_{K}$ or $b_{\beta}(a) \in L_{K}$ is finite whenever $r \neq 1$. If $b_{\alpha}(a) \in \mathcal{H} y p \backslash L_{K}\left(\right.$ resp. $\left.b_{\beta}(a) \in \mathcal{H} y p \backslash L_{K}\right)$, then $\left|x / q_{\alpha}(a)\right|<\epsilon^{\prime}\left(\operatorname{resp} .\left|y / q_{\beta}(a)\right|<\epsilon^{\prime}\right)$; see Lemma 3.12(2) (resp. Lemma 3.11(2)). Thus the proof of (1) is done. (Note that this is not true for $r=1$, since $\Delta \cap S_{\alpha}(1)=\Delta \cap S_{\beta}(1)=\Delta \cap S_{\gamma}(1)$.)

The proof of (2) is similar to that of (1).

Proof of Proposition 3.13 (1) By Lemma 2.15, one has

$$
\left(1-\left|\frac{x}{q_{\alpha}(a)}\right|-\left|\frac{y}{q_{\beta}(a)}\right|-\left|\frac{z}{q}\right|\right) \log \lambda(a)=\left(1-\left|\frac{x}{q_{\alpha}(a)}\right|-\left|\frac{y}{q_{\beta}(a)}\right|-\frac{1}{p+q}\right) \log \lambda(a) .
$$

Lemma 3.14 says that for any $\epsilon^{\prime}>0$, the inequality

$$
\left(1-\left|\frac{x}{q_{\alpha}(a)}\right|-\left|\frac{y}{q_{\beta}(a)}\right|-\frac{1}{p+q}\right) \log \lambda(a)>\left(1-\frac{1}{p+q}-2 \epsilon^{\prime}\right) \log \lambda(a)
$$

holds for any rational class $a=(x, y, z) \in \operatorname{int}(\Delta) \cap S_{\gamma}\left(\frac{p}{q}\right)$ but finitely many exceptions. If we set $a_{0}=\left[\frac{p}{2 p+2 q}, \frac{p}{2 p+2 q}\right]$, then by Lemma 3.2,

$$
\left(1-\frac{1}{p+q}\right) \log \lambda\left(a_{0}\right)=\min \operatorname{Ent}\left(N\left(\frac{p}{q}\right), \Omega_{S}\right) .
$$


For any $\epsilon>0$, choose a small number $\epsilon^{\prime}>0$ so that

$$
\left(1-\frac{1}{p+q}-2 \epsilon^{\prime}\right) \log \lambda\left(a_{0}\right)>\min \operatorname{Ent}\left(N\left(\frac{p}{q}\right), \Omega_{S}\right)-\epsilon .
$$

For any $a \in \operatorname{int}(\Delta) \cap S_{\gamma}\left(\frac{p}{q}\right)$, one has

$$
\begin{aligned}
\left(1-\frac{1}{p+q}-2 \epsilon^{\prime}\right) \log \lambda(a) & \geq\left(1-\frac{1}{p+q}-2 \epsilon^{\prime}\right) \log \lambda\left(a_{0}\right) \\
& >\min \operatorname{Ent}\left(N\left(\frac{p}{q}\right), \Omega_{S}\right)-\epsilon
\end{aligned}
$$

(see also Figure 10(left)). The proof of (1) is done.

(2) Let $a_{0}=\left[x_{0}, y_{0}\right]$ be the unique point of $\operatorname{int}(\Delta) \cap S_{\beta}\left(\frac{p}{q}\right)$ which enjoys

$$
\min \operatorname{Ent}\left(N\left(\frac{p}{q}\right), \Omega_{A}\right)=\left(1-\left|\frac{y_{0}}{q}\right|\right) \log \lambda\left(a_{0}\right) ;
$$

see Lemma 3.3. The function $1 / \log \lambda$ restricted to $\operatorname{int}(\Delta) \cap S_{\beta}\left(\frac{p}{q}\right)$ is strictly concave, and the entropy $\log \lambda(a)$ for $a \in \operatorname{int}(\Delta) \cap S_{\beta}\left(\frac{p}{q}\right)$ goes to $\infty$ as $a$ goes to a point on $\partial \Delta \cap S_{\beta}\left(\frac{p}{q}\right)$. This ensures the existence of $a_{i}=\left[x_{i}, y_{i}\right] \in \operatorname{int}(\Delta) \cap S_{\beta}\left(\frac{p}{q}\right)$ for $i \in\{-1,1\}$ satisfying the following:

- $\log \lambda\left(a_{-1}\right)=\log \lambda\left(a_{1}\right)$, and $0<y_{-1}<y_{0}<y_{1}<1$.

- $\left(1-\frac{1}{|q|}\right) \log \lambda\left(a_{i}\right)>\min \operatorname{Ent}\left(N\left(\frac{p}{q}\right), \Omega_{A}\right)$ for $i \in\{-1,1\}$.

For any $\epsilon>0$, one can choose a small number $\epsilon^{\prime}>0$ such that for $i \in\{-1,1\}$,

$$
\begin{aligned}
\epsilon & >2 \epsilon^{\prime} \log \lambda\left(a_{i}\right), \\
\left(1-\frac{1}{|q|}-2 \epsilon^{\prime}\right) \log \lambda\left(a_{i}\right) & >\min \operatorname{Ent}\left(N\left(\frac{p}{q}\right), \Omega_{A}\right) .
\end{aligned}
$$

Then for any $a=[x, y] \in \operatorname{int}(\Delta) \cap S_{\beta}\left(\frac{p}{q}\right)$ but finitely many exceptions, one has the following by a consequence of Lemma 3.14: if either $y>y_{1}$ or $y<y_{-1}$, then

$$
\begin{aligned}
\left(1-\left|\frac{x}{q_{\alpha}(a)}\right|-\left|\frac{y}{q}\right|-\frac{z}{q_{\gamma}(a)}\right) \log \lambda(a) & >\left(1-\frac{1}{|q|}-2 \epsilon^{\prime}\right) \log \lambda(a) \\
& >\left(1-\frac{1}{|q|}-2 \epsilon^{\prime}\right) \log \lambda\left(a_{ \pm 1}\right) \\
& >\min \operatorname{Ent}\left(N\left(\frac{p}{q}\right), \Omega_{A}\right) \\
& >\min \operatorname{Ent}\left(N\left(\frac{p}{q}\right), \Omega_{A}\right)-\epsilon .
\end{aligned}
$$


If $y_{-1} \leq y \leq y_{1}$, then one has $\log \lambda\left(a_{i}\right) \geq \log \lambda(a)$ for $i \in\{-1,1\}$. Thus

$$
\begin{aligned}
\left(1-\left|\frac{x}{q_{\alpha}(a)}\right|-\left|\frac{y}{q}\right|-\frac{z}{q_{\gamma}(a)}\right) \log \lambda(a) & >\left(1-\left|\frac{y}{q}\right|-2 \epsilon^{\prime}\right) \log \lambda(a) \\
& >\left(1-\left|\frac{y}{q}\right|\right) \log \lambda(a)-2 \epsilon^{\prime} \log \lambda\left(a_{ \pm 1}\right) \\
& \geq \min \operatorname{Ent}\left(N\left(\frac{p}{q}\right), \Omega_{A}\right)-2 \epsilon^{\prime} \log \lambda\left(a_{ \pm 1}\right) \\
& >\min \operatorname{Ent}\left(N\left(\frac{p}{q}\right), \Omega_{A}\right)-\epsilon .
\end{aligned}
$$

The third inequality comes from Lemma 3.3 (see also Figure 10(right)). This completes the proof of (2).
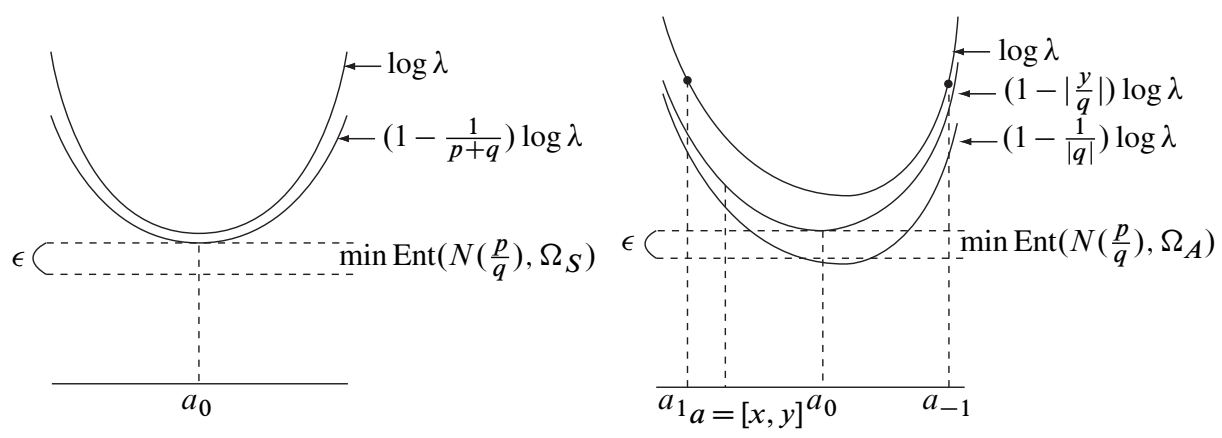

Figure 10: $\log \lambda$ and $(1-1 /(p+q)) \log \lambda$ on $\operatorname{int}(\Delta) \cap S_{\gamma}\left(\frac{p}{q}\right)$ (left); $\log \lambda$, $(1-|y / q|) \log \lambda$ and $(1-1 /|q|) \log \lambda$ on $\operatorname{int}(\Delta) \cap S_{\beta}\left(\frac{p}{q}\right)$ (right).

We are now ready to prove Theorem 1.4(1).

Proof of Theorem 1.4(1) We start by expressing Theorem 1.3 with [19, Lemma 4.8] in the following way.

Claim 3.15 Let $r \in\left\{-4, \frac{3}{-2}, \frac{1}{-2}, 2\right\}$. For each $g \geq 3$, there exists a primitive fibered class $h_{g}(r) \in H_{2}(N, \partial N)$ with the following properties.

- One of the boundary slopes of $h_{g}(r)$, say $b_{\beta}\left(h_{g}(r)\right)$ equals $r$, ie, $h_{g}(r) \in S_{\beta}(r)$.

- $\phi_{h_{g}(r)}$ is a mapping class on a surface of genus $g$ such that $\phi_{h_{g}(r)} \in \mathcal{M}$ for large $g$, and

$$
\lim _{g \rightarrow \infty} g \log \lambda\left(\widehat{\phi}_{h_{g}(r)}\right)=\log \left(\frac{3+\sqrt{5}}{2}\right) .
$$

In other words $\lim _{g \rightarrow \infty} \operatorname{Ent}\left(\hat{\phi}_{h_{g}(r)}\right)=2 \log \left(\frac{3+\sqrt{5}}{2}\right)$. 
Recall that $a_{g}$ is a primitive fibered class of $H_{2}(N, \partial N)$ such that $\phi_{a_{g}} \in \mathcal{M}$ and $\hat{\delta}_{g}$ is achieved by $\widehat{\phi}_{a_{g}} \in \widehat{\mathcal{M}} \cap \operatorname{Mod}\left(\Sigma_{g}\right)$. Lemma 3.9, Proposition 3.10 and Claim 3.15 tell us that for large $g$, one of the boundary slopes of $a_{g}$ is an element of the finite set $L_{K_{0}}$. No boundary slopes of $a_{g}$ equal 1 when $g \geq 2$. (We will prove Lemma 3.28 which implies this fact.) Thus for large $g$, one of the boundary slopes of $a_{g}$ is an element of $L_{K_{0}} \backslash\{1\}$.

We prove that for large $g$, one of the boundary slopes of $a_{g}$ must be $-4, \frac{3}{-2}, \frac{1}{-2}$ or 2. We fix $\epsilon>0$ so that we have $1.97475-\epsilon>2 \log ((3+\sqrt{5}) / 2)$. Now we set $L_{K_{0}}^{\prime}=L_{K_{0}} \backslash\left\{-4, \frac{3}{-2}, \frac{1}{-2}, 1,2\right\}$. Let $a \in H_{2}(N, \partial N)$ be a primitive fibered class such that one of the boundary slopes of $a$ is an element of $L_{K_{0}}^{\prime}$ and $\left\{b_{\alpha}(a), b_{\beta}(a), b_{\gamma}(a)\right\} \cap$ $\left\{-4, \frac{3}{-2}, \frac{1}{-2}, 1,2\right\}=\varnothing$. Suppose that $\phi_{a} \in \mathcal{M}$. Then Theorem 1.11 (or Theorem 3.1) implies that

$\min \operatorname{Ent}\left(N\left(b_{\alpha}(a)\right)\right), \min \operatorname{Ent}\left(N\left(b_{\beta}(a)\right)\right), \min \operatorname{Ent}\left(N\left(b_{\gamma}(a)\right)\right)>1.97475$.

It follows that $\operatorname{Ent}\left(\widehat{\phi}_{a}\right)>1.97475-\epsilon$ for any such a class $a$ but finitely many exceptions, which is ensured by Lemma 3.9 and Proposition 3.13. Thus for large $g$, one of the boundary slopes of $a_{g}$ must be an element of $\left\{-4, \frac{3}{-2}, \frac{1}{-2}, 2\right\}$.

Again by Proposition 3.13, the set of normalized entropies of mapping classes $\widehat{\phi}_{a} \in \widehat{\mathcal{M}}$ such that $\left\{b_{\alpha}(a), b_{\beta}(a), b_{\gamma}(a)\right\} \cap\left\{-4, \frac{3}{-2}, \frac{1}{-2}, 2\right\} \neq \varnothing$ have no accumulation values less than $2 \log ((3+\sqrt{5}) / 2)$. This together with Claim 3.15 leads to the conclusion.

\subsection{Proof of Theorem 1.4(2)}

For $r \in \mathcal{H} y p$, let $\widehat{\delta}_{g}(r)$ be minimum among dilatations of elements $\widehat{\phi}_{a} \in \widehat{\mathcal{M}} \cap \operatorname{Mod}\left(\Sigma_{g}\right)$ such that $a \in S_{\beta}(r)$ and $\phi_{a} \in \mathcal{M}$. We set $\hat{\delta}_{g}(r)=\infty$ when there exist no such elements. Clearly we have $\delta_{g} \leq \hat{\delta}_{g} \leq \hat{\delta}_{g}(r)$.

The proof of Theorem 1.4(1) implies that for large $g, \widehat{\delta}_{g}$ is either $\widehat{\delta}_{g}\left(\frac{3}{-2}\right), \widehat{\delta}_{g}\left(\frac{1}{-2}\right)$ or $\widehat{\delta}_{g}(2)$, because $N(-4) \simeq N\left(\frac{3}{-2}\right)$. We prove the following.

Proposition 3.16 $\min \left\{\widehat{\delta}_{g}\left(\frac{3}{-2}\right), \widehat{\delta}_{g}\left(\frac{1}{-2}\right), \widehat{\delta}_{g}(2)\right\}<\widehat{\delta}_{g}(2)$ for each $g \geq 4$.

Proof Set $r=\frac{3}{-2}$ and $r^{\prime}=-2-r=\frac{1}{-2}$. Recall that $\lambda_{(k, \ell)}$ is the largest real root of the polynomial $f_{(k, \ell)}(t)$ as in Section 1.7. Let $k$ and $\ell$ be coprime integers such that $0<\ell<k$. By the discussion in Section 1.7, we see that

$$
\lambda\left(k \overline{\mathfrak{a}}_{r} \pm \ell \overline{\mathfrak{b}}_{r}\right)=\lambda\left(k \overline{\mathfrak{a}}_{r^{\prime}} \pm \ell \overline{\mathfrak{b}}_{r^{\prime}}\right)=\lambda_{(k, \ell)} .
$$


The number $\widehat{\delta}_{g}\left(\frac{1}{-2}\right)$ was computed in [14, Theorem 1.4]:

$$
\begin{aligned}
& \widehat{\delta}_{g}\left(\frac{1}{-2}\right)=\lambda_{(g+1,3)} \quad \text { if } g \equiv 0,1,3,4(\bmod 6), g \geq 3, \\
& \hat{\delta}_{g}\left(\frac{1}{-2}\right)=\lambda_{(g+1,1)} \quad \text { if } g \equiv 2,5(\bmod 6), g \geq 5 .
\end{aligned}
$$

The following inequalities were proved in [19, Proposition 4.26]:

$$
\begin{aligned}
& \widehat{\delta}_{g}\left(\frac{3}{-2}\right)<\widehat{\delta}_{g}\left(\frac{1}{-2}\right) \quad \text { for } g \equiv 0,1,5,6,7,9(\bmod 10), g \geq 5, \\
& \widehat{\delta}_{g}\left(\frac{1}{-2}\right)<\widehat{\delta}_{g}\left(\frac{3}{-2}\right) \quad \text { for } g \equiv 3,8(\bmod 10), g \geq 3 .
\end{aligned}
$$

Thus it suffices to prove that $\widehat{\delta}_{g}(2)>\widehat{\delta}_{g}\left(\frac{1}{-2}\right)$ for each $g \geq 4$.

Let $a$ be a fibered class of $S_{\beta}(2)$ such that $\phi_{a} \in \mathcal{M}$ and $\widehat{\phi}_{a} \in \widehat{\mathcal{M}} \cap \operatorname{Mod}\left(\Sigma_{g}\right)$ for $g \geq 3$. Then $\lambda(a)=\lambda_{(g, \ell)}$ for some $1 \leq \ell<g$; see [19, Lemma 4.1]. There exists such a class $a$ whose dilatation $\lambda(a)$ equals $\lambda_{(g, 1)}$. This is proved by [19, Remark 4.18] together with the monodromy $\Phi_{g \mathfrak{r}+1 \mathfrak{s}}$ (in the notation of [19]). The inequality $\lambda_{(k, \ell)}<\lambda_{(k, \ell+1)}$ for $1<\ell+1<k$ (see [19, Lemma 4.15]) gives the equality $\widehat{\delta}_{g}(2)=\lambda_{(g, 1)}$ for $g \geq 3$.

It holds that $\lambda_{(g, 1)}>\lambda_{(g+1,1)}$ for $g \geq 2$; see [14, Proposition 4.3]. Hence we have $\hat{\delta}_{g}(2)>\hat{\delta}_{g}\left(\frac{1}{-2}\right)$ for $g \equiv 2,5(\bmod 6)$. We use the following claim to prove $\hat{\delta}_{g}(2)>\hat{\delta}_{g}\left(\frac{1}{-2}\right)$ for other cases.

Claim 3.17 [19, Proposition 4.17] If $\lambda_{(k+1, \ell)}<\lambda_{(k, 1)}$ for some $k \geq \ell \geq 2$, then

$$
\lambda_{(k+2, \ell)}<\lambda_{(k+1,1)} \text {. }
$$

One can check that $\lambda_{(4,1)} \approx 1.2806>\lambda_{(5,3)} \approx 1.2612$. Thus $\lambda_{(g, 1)}>\lambda_{(g+1,3)}$ for all $g \geq 4$. This implies that $\hat{\delta}_{g}(2)>\hat{\delta}_{g}\left(\frac{1}{-2}\right)$ for $g \equiv 0,1,3,4(\bmod 6)$.

Remark 3.18 From the proof of Proposition 3.16, we see the following: for large $g$ such that $g \equiv 0,1,5,6,7,9(\bmod 10)($ resp. such that $g \equiv 3,8(\bmod 10))$, $\widehat{\delta}_{g}$ is achieved by the monodromy of some $\Sigma_{g}$-bundle over the circle obtained from $N\left(\frac{3}{-2}\right)$ (resp. $\left.N\left(\frac{1}{-2}\right)\right)$ by Dehn filling both cusps. For many $g$ such that $g \equiv 2,4(\bmod 10)$, we have $\widehat{\delta}_{g}\left(\frac{3}{-2}\right)<\widehat{\delta}_{g}\left(\frac{1}{-2}\right)$; see [19, Proposition 4.28]. It might be true $\widehat{\delta}_{g}\left(\frac{3}{-2}\right)<\widehat{\delta}_{g}\left(\frac{1}{-2}\right)$ holds for all $g \equiv 2,4(\bmod 10)$; see [19, Question 4.32]. 


\subsection{Proofs of Theorems 1.5, 1.6, 1.7 and 1.10}

First, we prove that there exists an element of $\widehat{\mathcal{M}}^{+}$defined on $\Sigma_{g}$.

Lemma 3.19 For $g \geq 2, \widehat{\mathcal{M}}^{+} \cap \operatorname{Mod}\left(\Sigma_{g}\right) \neq \varnothing$.

We recall the following.

Lemma 3.20 [19, Proposition 3.5] The mapping class $\phi_{(x, y, z)}$ associated to a primitive fibered class $(x, y, z) \in \operatorname{int}\left(C_{\Delta}\right)$ has orientable invariant foliations if and only if $x$ and $y$ are even and $z$ is odd.

Proof of Lemma 3.19 For $g \geq 2$ even, let $u_{g}=(g, g,-1) \in \operatorname{int}\left(C_{\Delta}\right)$. For $g \geq 3$ odd, let $u_{g}=(g+1, g+1,1) \in \operatorname{int}\left(C_{\Delta}\right)$. The minimal representative $F_{u_{g}}$ is a genus $g$ surface with 3 boundary components. By Lemma 3.20, we see that $\phi_{u_{g}} \in \mathcal{M}$, since $\phi_{u_{g}}$ has orientable invariant foliations. In particular $\widehat{\phi}_{u_{g}} \in \widehat{\mathcal{M}}^{+} \cap \operatorname{Mod}\left(\Sigma_{g}\right)$.

From the proof of Lemma 3.19, we have that $\delta_{g}^{+} \leq \lambda_{(g, g,-1)}$ for $g$ even, and $\lambda_{(g, g,-1)}$ is the largest real root of $f_{(g, g,-1)}(t)=t^{2 g+1}-2 t^{g+1}-2 t^{g}+1$. Thus we have $\widehat{\phi}_{u_{g}} \in \widehat{\mathcal{M}}^{+} \cap \operatorname{Mod}\left(\Sigma_{g}\right)$ have the same dilatation as examples by Minakawa and Hironaka and Kin; see [27; 15].

Next, we recall upper bounds on $\delta_{g}^{+}$when $g \not \equiv 0(\bmod 6)$ by Hironaka, Aaber and Dunfield, and Kin and Takasawa which are sharper than the bound $\delta_{g}^{+} \leq \lambda_{(g, g,-1)}$. To do this, let us define $\hat{\delta}_{g}^{+}(r)$ for $r \in \mathcal{H} y p$. Let $\hat{\delta}_{g}^{+}(r)$ be the minimum among dilatations of elements $\widehat{\phi}_{a} \in \widehat{\mathcal{M}}^{+} \cap \operatorname{Mod}\left(\Sigma_{g}\right)$ such that $a \in S_{\beta}(r)$ and $\phi_{a} \in \mathcal{M}$. We set $\hat{\delta}_{g}^{+}(r)=\infty$ when there exist no such elements. Clearly $\delta_{g}^{+} \leq \hat{\delta}_{g}^{+} \leq \hat{\delta}_{g}^{+}(r)$.

Lemma 3.21 (1) $\hat{\delta}_{g}^{+}\left(\frac{3}{-2}\right)=\widehat{\delta}_{g}^{+}\left(\frac{1}{-2}\right)=\widehat{\delta}_{g}^{+}(2)=\infty$ if $g \equiv 0(\bmod 6)$.

(2) $\hat{\delta}_{g}^{+}\left(\frac{1}{-2}\right)=\lambda_{(g, 1)}, \hat{\delta}_{g}^{+}\left(\frac{3}{-2}\right)=\widehat{\delta}_{g}^{+}(2)=\infty$ if $g \equiv 2,4(\bmod 6)$.

(3) $\min \left\{\hat{\delta}_{g}^{+}\left(\frac{3}{-2}\right), \hat{\delta}_{g}^{+}\left(\frac{1}{-2}\right), \hat{\delta}_{g}^{+}(2)\right\}=\hat{\delta}_{g}^{+}\left(\frac{3}{-2}\right)=\lambda_{(g+2,4)}$ if $g \equiv 1,5(\bmod 10)$.

(4) $\min \left\{\hat{\delta}_{g}^{+}\left(\frac{3}{-2}\right), \hat{\delta}_{g}^{+}\left(\frac{1}{-2}\right), \hat{\delta}_{g}^{+}(2)\right\}=\widehat{\delta}_{g}^{+}\left(\frac{1}{-2}\right)=\lambda_{(g+1,3)}$ if $g \equiv 3(\bmod 10)$ and $g \equiv 1,3(\bmod 6)$.

(5) $\min \left\{\hat{\delta}_{g}^{+}\left(\frac{3}{-2}\right), \hat{\delta}_{g}^{+}\left(\frac{1}{-2}\right), \hat{\delta}_{g}^{+}(2)\right\}=\widehat{\delta}_{g}^{+}\left(\frac{1}{-2}\right)=\lambda_{(g+1,1)}$ if $g \equiv 3(\bmod 10)$ and $g \equiv 5(\bmod 6)$.

(6) $\min \left\{\hat{\delta}_{g}^{+}\left(\frac{3}{-2}\right), \hat{\delta}_{g}^{+}\left(\frac{1}{-2}\right), \hat{\delta}_{g}^{+}(2)\right\}=\widehat{\delta}_{g}^{+}\left(\frac{3}{-2}\right)=\lambda_{(g+2,2)}$ if $g \equiv 7,9(\bmod 10)$. 
Proof We have $\hat{\delta}_{g}^{+}\left(\frac{3}{-2}\right)=\hat{\delta}_{g}^{+}(2)=\infty$ if $g$ is even [19, Corollary 4.5, Lemma 4.11]. As a consequence of [14], $\hat{\delta}_{g}^{+}(1 /(-2))=\infty$ if $g \equiv 0(\bmod 6)$. By [14, Theorem 1.5], $\hat{\delta}_{g}^{+}(1 /(-2))=\lambda_{(g, 1)}$ if $g \equiv 2,4(\bmod 6)$. This completes the proofs of $(1)$ and $(2)$.

By using the same argument as in Proposition 3.16, one can prove that

$$
\min \left\{\hat{\delta}_{g}^{+}\left(\frac{3}{-2}\right), \hat{\delta}_{g}^{+}\left(\frac{1}{-2}\right), \hat{\delta}_{g}^{+}(2)\right\}<\hat{\delta}_{g}^{+}(2) \quad \text { if } g \text { is odd. }
$$

This together with [19, Propositions 4.23, 4.34] implies the claims (3)-(6).

If we fix $\ell>0$, then $k \log \lambda_{(k, \ell)}$ and $k \log \lambda_{(k, k,-1)}$ go to $\log ((3+\sqrt{5}) / 2)$ and $\log (2+\sqrt{3})$ respectively if $k$ goes to $\infty$. Thus the upper bound on $\delta_{g}^{+}$in Lemma 3.21 when $g \not \equiv 0(\bmod 6)$ is sharper than the bound $\delta_{g}^{+} \leq \lambda_{(g, g,-1)}$ for large $g$.

Putting all things together, we have the upper bound on $\delta_{g}^{+}$due to $[14 ; 1 ; 19 ; 27 ; 15]$ :

- $\delta_{g}^{+} \leq \lambda_{(g, g,-1)}$ when $g \equiv 0(\bmod 6)$,

- $\delta_{g}^{+} \leq \lambda_{(g, 1)}$ when $g \equiv 2,4(\bmod 6)$,

and for $g$ odd,

- $\delta_{g}^{+} \leq \lambda_{(g+2,4)}$ when $g \equiv 1,5(\bmod 10)$,

- $\delta_{g}^{+} \leq \lambda_{(g+1,3)}$ when $g \equiv 3(\bmod 10)$ and $g \equiv 1,3(\bmod 6)$,

- $\delta_{g}^{+} \leq \lambda_{(g+1,1)}$ when $g \equiv 3(\bmod 10)$ and $g \equiv 5(\bmod 6)$,

- $\delta_{g}^{+} \leq \lambda_{(g+2,2)}$ when $g \equiv 7,9(\bmod 10)$.

Proof of Theorem 1.5 The proof of the claim (1) is similar to that of Theorem 1.4(1). The claims (2), (3) hold by Lemma 3.21(2)-(6) and by the same argument as in the proof of Theorem 1.4(2).

We are ready to prove Theorem 1.10 .

Proof of Theorem 1.10 Let $a_{g}^{+}$be a primitive fibered class of $H_{2}(N, \partial N)$ such that $\phi_{a_{g}^{+}} \in \mathcal{M}$ and $\hat{\delta}_{g}^{+}$is achieved by $\widehat{\phi}_{a_{g}^{+}} \in \widehat{\mathcal{M}}^{+} \cap \operatorname{Mod}\left(\Sigma_{g}\right)$. We prove the claim (2) first.

(2) Suppose that $g \equiv 2,4(\bmod 6)$. By Lemma 3.21(2), we have $\hat{\delta}_{g}^{+}\left(\frac{1}{-2}\right)=\lambda_{(g, 1)}$. By Theorem 1.5(2), the fibered class $a_{g}^{+}$must have a boundary slope $\frac{1}{-2}$ for large $g$. Thus $\hat{\delta}_{g}^{+}=\hat{\delta}_{g}^{+}\left(\frac{1}{-2}\right)=\lambda_{(g, 1)}$. 
(1) Suppose that $g \equiv 0(\bmod 6)$. By Lemma 3.21(1), no boundary slope of $a_{g}^{+}$ is an element of $\left\{-4, \frac{3}{-2}, \frac{1}{-2}, 2\right\}$. From the proof of Theorem 1.4(1), we know that $\operatorname{Ent}\left(\widehat{\phi}_{a_{g}^{+}}\right)>1.97475-\epsilon$ for any $g \equiv 0(\bmod 6)$ but finitely many exceptions. Thus

$$
\operatorname{Ent}\left(\widehat{\phi}_{a_{g}^{+}}\right)=\left|\chi\left(\Sigma_{g}\right)\right| \log \hat{\delta}_{g}^{+}>1.97475-\epsilon>2 \log \left(\frac{3+\sqrt{5}}{2}\right)
$$

for large $g$ such that $g \equiv 0(\bmod 6)$. Since we have $\lim _{g \rightarrow \infty}\left|\chi\left(\Sigma_{g}\right)\right| \log \lambda_{(g, 1)}=$ $2 \log ((3+\sqrt{5}) / 2)$, the inequality $\hat{\delta}_{g}^{+}>\lambda_{(g, 1)}$ holds for such a large $g$.

We now prove Theorem 1.6 which improves the upper bound (1-5) in Section 1.5. To do this, it suffices to prove the following two lemmas.

Lemma 3.22 $\min \operatorname{Ent}\left(N(-6), \Omega_{S}\right)=\min \operatorname{Ent}\left(N(4), \Omega_{S}\right)=4 \log \delta\left(D_{5}\right)$.

Proof We have that both minimal dilatations $\delta_{2}$ and $\delta\left(D_{5}\right)$ are the largest real roots of $t^{5}-2 t^{3}-2 t^{2}+1$; see [5; 12]. By Lemma 3.2,

$$
\min \operatorname{Ent}\left(N(4), \Omega_{S}\right)=\frac{4}{5} \log \lambda_{[2 / 5,2 / 5]}=4 \log \lambda_{(2,2,-1)} \text {. }
$$

Since $f_{(2,2,-1)}(t)=t^{5}-2 t^{3}-2 t^{2}+1$, we have the identities $\lambda_{(2,2,-1)}=\delta_{2}=\delta\left(D_{5}\right)$. By Theorem 2.26(1), it follows that $\left(N(4), \Omega_{S}\right) \underset{\text { ent }}{\sim}\left(N(-6), \Omega_{S}\right)$. Hence

$$
\min \operatorname{Ent}\left(N(4), \Omega_{S}\right)=\min \operatorname{Ent}\left(N(-6), \Omega_{S}\right) .
$$

This completes the proof.

Lemma 3.23 For each $i \geq 0$, there exists a $\Sigma_{6+12 i}$-bundle over the circle which satisfies the following. It is obtained from $N(4)$ by Dehn filling both cusps along boundary slopes of a fiber of $N(4)$, and the monodromy $\Phi_{i}: \Sigma_{6+12 i} \rightarrow \Sigma_{6+12 i}$ of the fibration has orientable invariant foliations. Moreover

$$
\min \operatorname{Ent}\left(N(4), \Omega_{S}\right)=\lim _{i \rightarrow \infty} \operatorname{Ent}\left(\Phi_{i}\right)
$$

Proof Consider a primitive fibered class

$$
a_{q}=(4 q+8,4 q+4,-2 q-3) \in \operatorname{int}\left(C_{\Delta}\right) \cap S_{\gamma}(4) \text { for } q \geq 0 .
$$

Lemma 3.20 tells us that the monodromy of the fibration on $N$ associated to $a_{q}$ has orientable invariant foliations. In particular, $\phi_{a_{q}} \in \mathcal{M}$ and $\widehat{\phi}_{a_{q}} \in \widehat{\mathcal{M}}^{+}$. Now let $q=3 i$ for $i \geq 0$. Then the numbers of the boundary components of $F_{a_{q}}$ lying on $T_{\alpha}, T_{\beta}$ and $T_{\gamma}$ are given by $1,1,2 q+3$ respectively (see Lemma 2.1), and the genus of $F_{a_{q}}$ is equal to $6+12 i$. 
The ray of $\bar{a}_{q} \in H_{2}(N(4), \partial N((4)))$ goes to the ray of $\overline{(2,2,-1)} \in H_{2}(N(4), \partial N((4)))$ as $q$ goes to $\infty$. Hence

$$
\operatorname{Ent}_{4}(\overline{(2,2,-1)})=\lim _{q \rightarrow \infty} \operatorname{Ent}_{4}\left(\bar{a}_{q}\right)
$$

On the other hand by Lemma 3.22, we have

$$
\min \operatorname{Ent}\left(N(4), \Omega_{S}\right)=4 \log \lambda_{(2,2,-1)}=\operatorname{Ent}_{4}(\overline{(2,2,-1)}) .
$$

Since the number of the boundary components of $F_{\bar{a}_{q}}$ is bounded (in fact, it is exactly 2), it follows that

$$
\operatorname{Ent}_{4}(\overline{(2,2,-1)})=\lim _{q \rightarrow \infty} \operatorname{Ent}_{4}\left(\bar{a}_{q}\right)=\lim _{q \rightarrow \infty} \operatorname{Ent}\left(\widehat{\phi}_{a_{q}}\right) .
$$

This completes the proof.

Proof of Theorem 1.7 In the proof of Lemma 3.23, we proved that for $g \equiv 6(\bmod 12)$ (if and only if $g \equiv 6,18,30,42,54,66,78(\bmod 84)$ ),

$$
\delta_{g}^{+} \leq \lambda\left(g+2, g-2,-\frac{g}{2}\right) .
$$

Let us prove that monodromies of the fibrations on $N(-6)$ give sharper upper bounds on $\delta_{g}^{+}$for some $g$. Let

$$
a_{q}^{\prime}=(6 q+4,6 q+2,2 q+1) \in \operatorname{int}\left(C_{\Delta}\right) \cap S_{\gamma}(-6) \text { for } q \geq 1 .
$$

The monodromy of the fibration on $N$ associated to $a_{q}^{\prime}$ has orientable invariant foliations by Lemma 3.20. Hence we have that $\phi_{a_{q}^{\prime}} \in \mathcal{M}$ and $\widehat{\phi}_{a_{q}^{\prime}} \in \widehat{\mathcal{M}}^{+}$. For $g \equiv 6,30,42,54,78(\bmod 84)$ and $g>0$, we set $q=(g-2) / 4$. Then the numbers of the boundary components of $F_{a_{q}^{\prime}}$ lying on $T_{\alpha}, T_{\beta}$ and $T_{\gamma}$ are given by $1,1,2 q+1$ respectively. The genus of $F_{a_{q}^{\prime}}$ is equal to $g$. Thus we have

$$
\delta_{g}^{+} \leq \lambda\left(\frac{3 g}{2}+1, \frac{3 g}{2}-1, \frac{g}{2}\right) .
$$

To check that this bound is sharper than the one above, we now prove the inequality

$$
\lambda\left(\frac{3 g}{2}+1, \frac{3 g}{2}-1, \frac{g}{2}\right)<\lambda\left(g+2, g-2,-\frac{g}{2}\right) .
$$

Recall that $\left(N(4), \Omega_{S}\right)$ and $\left(N(-6), \Omega_{S}\right)$ are entropy equivalent, and min Ent is attained by $\overline{(2,2,-1)}$ for $N(4)$ (resp. $\overline{(3,3,1)}$ for $N(-6)$ ). We note that the ray of $\bar{a}_{q^{\prime}} \in H_{2}(N(-6), \partial N((-6)))$ goes to the ray of $\overline{(3,3,1)} \in H_{2}(N(-6), \partial N((-6)))$ as $q$ goes to $\infty$. We have the identity on the Thurston norm,

$$
\left\|\overline{\left(\frac{3 g}{2}+1, \frac{3 g}{2}-1, \frac{g}{2}\right)}\right\|_{-6}=\left\|\overline{\left(g+2, g-2,-\frac{g}{2}\right)}\right\|_{4} \text {. }
$$


One can check that the ray of $\overline{\left(\frac{3 g}{2}+1, \frac{3 g}{2}-1, \frac{g}{2}\right)}$ is closer to the minimal ray than the ray of $\overline{\left(g+2, g-2,-\frac{g}{2}\right)}$. Because of the strict concavity of $1 /$ ent, we have the desired inequality.

Let us turn to the case $g \equiv 0(\bmod 12)$. We have not obtained an explicit upper bound on $g \log \delta_{g}^{+}$or $\delta_{g}^{+}$as in Theorem 1.6 or 1.7 in this case. However for many such $g$, we improve the previous bound $\delta_{g}^{+} \leq \lambda_{(g, g,-1)}$; see Table 1 . We note that our bound $\delta_{12}^{+} \leq \lambda_{(12,20,3)}$ in Table 1 is given by the example which occurs as the monodromy of the fibration on a manifold obtained from $N\left(\frac{3}{-4}\right)$ by Dehn filling both cusps. In the left column of Table 1 , other upper bounds on $\delta_{g}^{+}$when $g \equiv 0(\bmod 12)$ are given by examples which occur as the monodromies of fibrations on manifolds obtained from $N\left(\frac{5}{-4}\right)$. By our computer experiments, it seems that $\hat{\delta}_{g}^{+}$is realized by the example obtained from $N\left(\frac{5}{-4}\right)$ by Dehn filling both cusps for any $g \equiv 0(\bmod 12)$ and $g>12$. We ask the following.

Question 3.24 Does there exist a primitive fibered class $b_{i} \in \operatorname{int}\left(C_{\Delta}\right) \cap S_{\beta}\left(\frac{5}{-4}\right)$ for large $i$ which enjoys the following?

- The minimal representative $F_{b_{i}}$ has genus $12 i$, and $\phi_{b_{i}}$ has orientable invariant foliations.

- $\min \operatorname{Ent}\left(N\left(\frac{5}{-4}\right)\right)=\lim _{i \rightarrow \infty} \operatorname{Ent}_{5 /(-4)}\left(\bar{b}_{i}\right)$.

Proposition 3.25 If Question 3.24 is true, then

$$
\limsup _{g \equiv 0(\bmod 12)} g \log \delta_{g}^{+} \leq \frac{1}{2} \min \operatorname{Ent}\left(N\left(\frac{5}{-4}\right)\right)<1.1466 .
$$

Proof The existence of primitive integral classes $b_{i}$ implies the left inequality. To see the right inequality, we take $a=(292,300,83) \in \operatorname{int}\left(C_{\Delta}\right) \cap S_{\beta}\left(\frac{5}{-4}\right)$. The Thurston norm of $\bar{a}$ equals $\|a\|-\operatorname{gcd}(300,375)=434$. Thus

$$
\min \operatorname{Ent}\left(N\left(\frac{5}{-4}\right)\right) \leq \operatorname{Ent}_{5 /(-4)}(\bar{a})=434 \log \lambda(a) \approx 2.2930 .
$$

\subsection{Proof of Theorem 1.8}

This subsection concerns the monodromies of fibrations on the Whitehead link exterior $N(1)$.

Proposition 3.26 We have that an $S$-face of $N(1)$ and an $A$-face of $N(1)$ are entropy equivalent. 
Proof For each $k, \ell \in \mathbb{N}$, the class $k \overline{\mathfrak{a}}_{1}+\ell \overline{\mathfrak{b}}_{1}$ (resp. $k \overline{\mathfrak{a}}_{1}-\ell \overline{\mathfrak{b}}_{1}$ ) is an element of $\operatorname{int}\left(C_{\Omega_{S}}\right)$ (resp. $\operatorname{int}\left(C_{\Omega_{A}}\right)$ ) (see Figure 9). Further $k \overline{\mathfrak{a}}_{1} \pm \ell \overline{\mathfrak{b}}_{1}$ have the same Thurston norm. Thus it suffices to show that $k \overline{\mathfrak{a}}_{1} \pm \ell \overline{\mathfrak{b}}_{1}$ have the same entropy. Figure 11 illustrates the projections of the Whitehead link. The minimal representatives of $\overline{\mathfrak{a}}_{1}$ and $\overline{\mathfrak{b}}_{1}$ are depicted as in Figure 11(a). One can check that all three oriented links in this figure are isotopic in $S^{3}$ to each other. In particular the two oriented links (b) and (c) are isotopic fixing the trivial component. This implies that there exists an involution $f: N(1) \rightarrow N(1)$. This involution induces an isomorphism $f_{*}: H_{2}(N(1), \partial N(1)) \rightarrow$ $H_{2}(N(1), \partial N(1))$ which sends $\overline{\mathfrak{a}}_{1}$ to itself and $\overline{\mathfrak{b}}_{1}$ to $-\overline{\mathfrak{b}}_{1}$. Because $f_{*}$ is induced by the involution on the manifold $N(1)$, the entropy of $k \overline{\mathfrak{a}}_{1}+\ell \overline{\mathfrak{b}}_{1}$ must be equal to that of $k \overline{\mathfrak{a}}_{1}-\ell \overline{\mathfrak{b}}_{1}$.

By Lemma 3.2, one sees that $\min \operatorname{Ent}\left(N(1), \Omega_{S}\right)=2 \log \delta\left(D_{4}\right)$. This together with Proposition 3.26 leads to $\min \operatorname{Ent}\left(N(1), \Omega_{A}\right)=2 \log \delta\left(D_{4}\right)$. Thus we obtain:

Corollary 3.27 $\min \operatorname{Ent}(N(1))=2 \log \delta\left(D_{4}\right) \approx 1.6628$

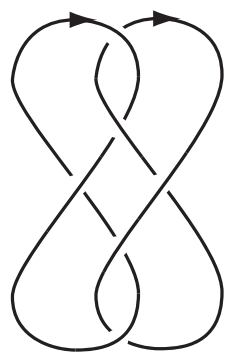

(a)

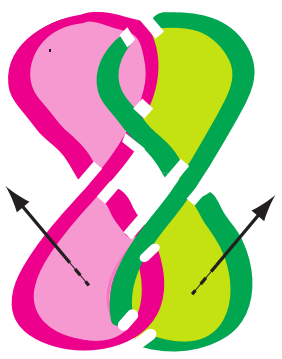

(b)

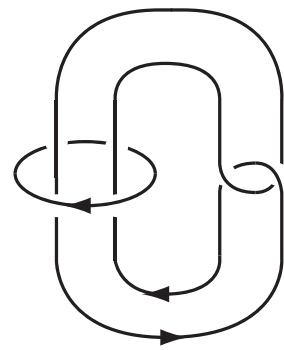

(c)

Figure 11: Projections of the Whitehead link [the minimal representatives of $\overline{\mathfrak{a}}_{1}$ and $\overline{\mathfrak{b}}_{1}$ are illustrated in (a)]

The following lemma is easy to verify by using Lemma 2.1 .

Lemma 3.28 The genus of each fiber of $N(1)$ equals 1. More precisely, for coprime integers $k, \ell \in \mathbb{N}$, the minimal representative of $k \overline{\mathfrak{a}}_{1}+\ell \overline{\mathfrak{b}}_{1}$ is a $(k+\ell)$-holed torus.

Remark 3.29 For $k$ and $\ell$ as in Lemma 3.28, the stable foliation of the monodromy $\Phi_{k \mathfrak{a}_{1}+\ell \mathfrak{b}_{1}}$ of the fibration on $N$ associated to $k \mathfrak{a}_{1}+\ell \mathfrak{b}_{1}$ has the following property. Each boundary component of the fiber $F_{k \mathfrak{a}_{1}+\ell \mathfrak{b}_{1}}$ lying on the torus specified by $\alpha, \beta$ and $\gamma$ has a 1 prong, 3 prongs and a 1 prong respectively. Hence $\phi_{k \mathfrak{a}_{1}+\ell \mathfrak{b}_{1}} \notin \mathcal{M}$.

For $n \geq 2$, let $\mathcal{W}_{n} \subset H_{2}(N(1), \partial N(1) ; \mathbb{Z})$ be the set of primitive fibered classes whose minimal representatives are $n$-holed tori. 
Proposition 3.30 The following class achieves the minimal dilatation among elements of $\mathcal{W}_{n}$.

(1) $\overline{\mathfrak{a}}_{1}+\overline{\mathfrak{b}}_{1}$ when $n=2$. Its dilatation equals the largest real root of

$$
f_{(1,1,-2)}(t)=t^{4}-2 t^{3}-2 t+1 \text {. }
$$

(2) $k \overline{\mathfrak{a}}_{1}+(k-1) \overline{\mathfrak{b}}_{1}$ when $n=2 k-1$ for $k \geq 2$. Its dilatation equals the largest real root of

$$
f_{(k, k-1,-2 k+1)}(t)=t^{4 k-2}-t^{3 k-1}-t^{3 k-2}-t^{k}-t^{k-1}+1 .
$$

(3) $(2 k+1) \overline{\mathfrak{a}}_{1}+(2 k-1) \overline{\mathfrak{b}}_{1}$ when $n=4 k$ for $k \geq 1$. Its dilatation equals the largest real root of

$$
f_{(2 k+1,2 k-1,-4 k)}(t)=t^{8 k}-t^{6 k+1}-t^{6 k-1}-t^{2 k+1}-t^{2 k-1}+1 .
$$

(4) $(2 k+3) \overline{\mathfrak{a}}_{1}+(2 k-1) \overline{\mathfrak{b}}_{1}$ when $n=4 k+2$ for $k \geq 1$. Its dilatation equals the largest real root of

$$
f_{(2 k+3,2 k-1,-4 k-2)}(t)=t^{8 k+4}-t^{6 k+5}-t^{6 k+1}-t^{2 k+3}-t^{2 k-1}+1 .
$$

Proof Proposition 3.26 says that for the study of monodromies of fibrations on $N(1)$, it is enough to deal with fibers whose homology classes are in the cone over an $S$-face. From the proof of Lemma 3.2, the center of $\Omega_{S}$ achieves $\min \operatorname{Ent}\left(N(1), \Omega_{S}\right)$. Then the proposition holds from the strict concavity of the function $\frac{1}{\mathrm{ent}_{1}}=\frac{1}{\log \lambda_{1}}: \operatorname{int}\left(C_{\Omega_{S}}\right) \rightarrow \mathbb{R}$ together with Lemma 3.28.

Lemma 3.31 Suppose $\frac{\ell}{k}$ goes to 1 as $k$ and $\ell$ go to $\infty$. Then $\left.\operatorname{Ent}_{1}\left(k \overline{\mathfrak{a}}_{1}+\ell \overline{\mathfrak{b}}_{1}\right)\right)$ goes to $\min \operatorname{Ent}(N(1))=2 \log \delta\left(D_{4}\right)$ as both $k$ and $\ell$ go to $\infty$.

Proof We have that $\min \operatorname{Ent}\left(N(1), \Omega_{S}\right)$ is achieved by the center of $\Omega_{S}$. This leads to the lemma.

Proof of Theorem 1.8 See Proposition 3.30 and Lemma 3.31.

\section{1-cusped manifolds with small volume}

The magic manifold plays a central role not only for the minimizing problem on dilatations but also for the minimizing problem on volumes of hyperbolic 3-manifolds. It was proved by Agol that the smallest volume among orientable 2-cusped hyperbolic 3 -manifold is achieved by either the Whitehead link exterior $N(1)$ or the Whitehead 
sister link exterior $N\left(\frac{3}{-2}\right)$; see Agol [2]. Gabai, Meyerhoff and Milley proved that 1-cusped orientable hyperbolic 3-manifolds with volume at most 2.848 are obtained from $N$ by Dehn filling two cusps, and they identified these 1-cusped manifolds, which we recall in Theorem 4.3. In the end of this section, we compute the normalized entropy of the monodromy of the fibration on each of them.

First we recall the following.

Theorem 4.1 (Gabai, Meyerhoff and Milley [11, Theorem 1.1]) Let $M$ be a $1-$ cusped orientable hyperbolic 3-manifold whose volume is at most 2.848. Then $M$ can be obtained from $M^{\prime}$ by Dehn filling all but one of the cusps, where $M^{\prime}$ is one of the 21 manifolds in the SnapPea census: $m 125, m 129, m 202, m 203, m 292, m 295$, $m 328, m 329, m 359, m 366, m 367, m 391, m 412, s 596, s 647, s 774, s 776, s 780$, $s 785, s 898, s 959$.

We have that $s 776$ is homeomorphic to the magic manifold $N$. All manifolds listed above other than $s 776$ have exactly 2 cusps. The 12 manifolds $m 125, m 129, \ldots, m 391$ are obtained from $s 776$ by Dehn filling a cusp. We compute the quantities min Ent etc. for these manifolds by using results in this paper; see Table 2. (One can check that the first column in Table 2 by using SnapPy [6].)

\begin{tabular}{|c|c|c|c|}
\hline manifold $M$ & $\min \operatorname{Ent}\left(M, \Omega_{S}\right)$ & $\min \operatorname{Ent}\left(M, \Omega_{A}\right)$ & $\min \operatorname{Ent}(M)$ \\
\hline$m 125 \simeq N\left(\frac{3}{-2}\right)$ & none & $2 \log \left(\frac{3+\sqrt{5}}{2}\right)$ & $2 \log \left(\frac{3+\sqrt{5}}{2}\right)$ \\
\hline$m 129 \simeq N(1)$ & $2 \log \lambda_{(1,1,-2)} \approx 1.6628$ & $2 \log \lambda_{(1,1,-2)}$ & $2 \log \lambda_{(1,1,-2)}$ \\
\hline$m 202 \simeq N\left(\frac{5}{-2}\right)$ & $?$ & $4 \log \lambda_{(4,2,1)} \approx 2.5318$ & \\
\hline$m 203 \simeq N\left(\frac{1}{-2}\right)$ & none & $2 \log \left(\frac{3+\sqrt{5}}{2}\right)$ & $2 \log \left(\frac{3+\sqrt{5}}{2}\right)$ \\
\hline$m 292 \simeq N(-5)$ & $6 \log \lambda_{(5,5,2)} \approx 2.0761$ & $?$ & \\
\hline$m 295 \simeq N(2)$ & $2 \log \left(\frac{3+\sqrt{5}}{2}\right)$ & $?$ & \\
\hline$m 328 \simeq N\left(\frac{4}{-3}\right)$ & none & as & \& \\
\hline$m 329 \simeq N\left(\frac{5}{-3}\right)$ & none & A & a \\
\hline$m 359 \simeq N\left(\frac{2}{-3}\right)$ & none & \& & is \\
\hline$m 366 \simeq N\left(\frac{7}{-2}\right)$ & $8 \log \lambda_{(7,7,4)} \approx 2.4181$ & $\diamond$ & $8 \log \lambda_{(7,7,4)}$ \\
\hline$m 367 \simeq N\left(\frac{1}{2}\right)$ & $4 \log \lambda_{(1,1,-4)} \approx 2.9314$ & $4 \log \lambda_{(4,2,1)} \approx 2.5318$ & $4 \log \lambda_{(4,2,1)}$ \\
\hline$m 391 \simeq N\left(\frac{1}{-3}\right)$ & none & A & A \\
\hline
\end{tabular}

Table 2: $\min$ Ent for some fibered 3-manifolds in Theorem 4.1 
Remark 4.2 We make comments on Table 2 .

(1) The filling slopes $\frac{p}{q}$ of $N\left(\frac{p}{q}\right)$ marked "?" do not enjoy the assumption of Theorem 3.1.

(2) By Theorem 2.26,

$$
N\left(\frac{5}{-3}\right) \underset{\text { ent }}{\sim} N\left(\frac{1}{-3}\right), \quad N\left(\frac{4}{-3}\right) \underset{\text { ent }}{\sim} N\left(\frac{2}{-3}\right) .
$$

This together with Lemma 3.5 implies that

$$
\begin{aligned}
\boldsymbol{\omega}=\min \operatorname{Ent}\left(N\left(\frac{5}{-3}\right)\right) & =\min \operatorname{Ent}\left(N\left(\frac{1}{-3}\right)\right) \\
& >\min \operatorname{Ent}\left(N\left(\frac{2}{-3}\right)\right) \\
& =\min \operatorname{Ent}\left(N\left(\frac{4}{-3}\right)\right)=\boldsymbol{s} .
\end{aligned}
$$

\begin{tabular}{|c|c|}
\hline $\begin{aligned} m 125 \simeq N\left(\frac{3}{-2}\right) \simeq S^{3} \backslash \operatorname{br}\left(T_{6,2}^{\prime}\right) \\
m 202 \simeq N\left(\frac{5}{-2}\right) \simeq S^{3} \backslash \operatorname{br}\left(T_{8,3}^{\prime}\right) \\
m 292 \simeq N(-5) \simeq S^{3} \backslash \operatorname{br}\left(T_{7,5}^{\prime}\right) \\
m 328 \simeq N\left(\frac{4}{-3}\right) \simeq S^{3} \backslash \operatorname{br}\left(T_{8,2}^{\prime}\right) \\
m 359 \simeq N\left(\frac{2}{-3}\right) \simeq S^{3} \backslash \operatorname{br}\left(T_{6,3}^{\prime}\right) \\
m 367 \simeq N\left(\frac{1}{2}\right) \\
s 776 \simeq N \simeq S^{3} \backslash \operatorname{br}\left(T_{6,3}\right)\end{aligned}$ & $\begin{aligned} & m 129 \simeq N(1) \\
& m 203 \simeq N\left(\frac{1}{-2}\right) \simeq S^{3} \backslash \operatorname{br}\left(T_{4,1}^{\prime}\right) \\
& m 295 \simeq N(2) \\
& m 329 \simeq N\left(\frac{5}{-3}\right) \simeq S^{3} \backslash \operatorname{br}\left(T_{9,5}^{\prime}\right) \\
& m 366 \simeq N\left(\frac{7}{-2}\right) \simeq S^{3} \backslash \operatorname{br}\left(T_{10,4}^{\prime}\right) \\
& m 391 \simeq N\left(\frac{1}{-3}\right) \simeq S^{3} \backslash \operatorname{br}\left(T_{5,1}^{\prime}\right)\end{aligned}$ \\
\hline
\end{tabular}

(3) We know $\min \operatorname{Ent}\left(N\left(\frac{7}{-2}\right), \Omega_{S}\right)=8 \log \lambda_{(7,7,4)} \approx 2.4181$ by Lemma 3.2, and

$$
\diamond=\min \operatorname{Ent}\left(N\left(\frac{7}{-2}\right), \Omega_{A}\right)>\min \operatorname{Ent}\left(N\left(\frac{5}{-2}\right), \Omega_{A}\right) \approx 2.5318
$$

by Lemmas 3.5 and 3.6(2). Thus, $\min \operatorname{Ent}\left(N\left(\frac{7}{-2}\right)\right)=8 \log \lambda(7,7,4)$.

Table 3: $s 776 \simeq N$ and manifolds obtained from $N$ by Dehn filling in Theorem 4.1

Now we would like to point out that many manifolds in Table 2 are braided link exteriors. To do this, we first recall the definition of the braided link. Let $B_{n}$ be the $n$-braid group, and let $\sigma_{1}, \sigma_{2}, \ldots, \sigma_{n-1} \in B_{n}$ be the Artin generators of $B_{n}$; see Figure 12 . The braided link $\operatorname{br}(b)$ of a braid $b$ is the union of the closed braid of $b$ and its axis; see Figure 13. For example, the link $\sigma_{2}^{2}$ is the braided link $b r\left(\sigma_{1}^{-1} \sigma_{2}\right)$; see Figure 1 . Let $T_{m, p}$ be the following $m$-braid for $m \geq 3$ and $p \geq 1$ :

$$
T_{m, p}=\left(\sigma_{1}^{2} \sigma_{2} \sigma_{3} \cdots \sigma_{m-1}\right)^{p} \sigma_{m-1}^{-2} .
$$


For example $T_{6,2}=\left(\sigma_{1}^{2} \sigma_{2} \sigma_{3} \sigma_{4} \sigma_{5}\right)^{2} \sigma_{5}^{-2}=\sigma_{1}^{2} \sigma_{2} \sigma_{3} \sigma_{4} \sigma_{5} \sigma_{1}^{2} \sigma_{2} \sigma_{3} \sigma_{4} \sigma_{5}^{-1}$; see Figure 14 . Forgetting the first strand of $T_{m, p}$, one obtains the $(m-1)$-braid, call it $T_{m, p}^{\prime}$. It was shown in [18, Corollary 3.2] that if $m-1$ and $p$ are coprime, then $N \simeq S^{3} \backslash b r\left(T_{m, p}\right)$. By [18, Theorem 3.4], one sees that if $x, y \in \mathbb{N}$ are coprime such that $y /(-x) \in \mathcal{H} y p$, then there exists $p(x, y) \in \mathbb{N}$ such that $N(y /(-x)) \simeq S^{3} \backslash \operatorname{br}\left(T_{x+y+1, p(x, y)}^{\prime}\right)$. Some manifolds in Table 2 can be described as the exterior of the braided link of the form $S^{3} \backslash \operatorname{br}\left(T_{m, p}^{\prime}\right)$; see Table 3. It is intriguing that some braids appearing in this table reach the minimal dilatations. Table 4 shows the minimal dilatation $\delta\left(D_{n}\right)$ and an $n$-braid (equivalently an element of $\operatorname{Mod}\left(D_{n}\right)$ ) realizing $\delta\left(D_{n}\right)$. Here $b \sim b^{\prime}$ means that $b$ is conjugate to $b^{\prime}$.

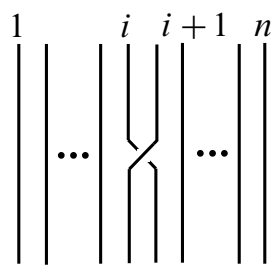

Figure 12: Braid $\sigma_{i} \in B_{n}$

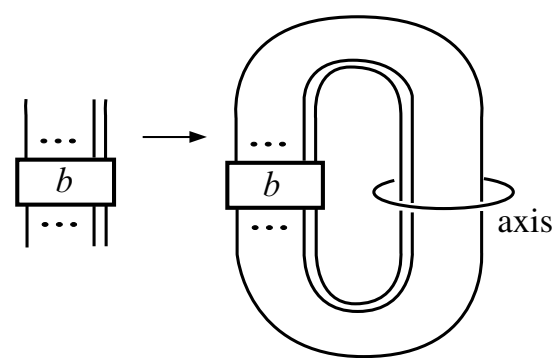

Figure 13: Braid $b \rightarrow$ braided link $\operatorname{br}(b)$

\begin{tabular}{|cccc|}
\hline$n$ & $\delta\left(D_{n}\right)$ & $n$-braid realizing $\delta\left(D_{n}\right)$ & reference \\
\hline 3 & $\frac{3+\sqrt{5}}{2} \approx 2.6180$ & $T_{4,1}^{\prime}=\sigma_{1} \sigma_{2}^{-1}$ & {$[25 ; 13]$} \\
4 & $\lambda_{(3,1,0)} \approx 2.2966$ & $T_{5,1}^{\prime}=\sigma_{1} \sigma_{2} \sigma_{3}^{-1}$ & {$[29 ; 12]$} \\
5 & $\lambda_{(2,3,0)} \approx 1.7220$ & $T_{6,2}^{\prime} \sim \sigma_{1} \sigma_{2} \sigma_{3} \sigma_{4} \sigma_{1} \sigma_{2}$ & {$[12]$} \\
6 & $\lambda_{(3,2,0)} \approx 1.7220$ & $T_{6,3} \sim\left(\sigma_{2} \sigma_{1} \sigma_{2} \sigma_{1}\left(\sigma_{1} \sigma_{2} \sigma_{3} \sigma_{4} \sigma_{5}\right)^{2}\right)^{-1}$ & {$[20]$} \\
7 & $\lambda_{(3,4,0)} \approx 1.4655$ & $T_{8,2}^{\prime} \sim \sigma_{4}^{-2}\left(\sigma_{1} \sigma_{2} \sigma_{3} \sigma_{4} \sigma_{5} \sigma_{6}\right)^{2}$ & {$[20]$} \\
8 & $\lambda_{(3,5,0)} \approx 1.4134$ & $T_{9,5}^{\prime} \sim \sigma_{2}^{-1} \sigma_{1}^{-1}\left(\sigma_{1} \sigma_{2} \sigma_{3} \sigma_{4} \sigma_{5} \sigma_{6} \sigma_{7}\right)^{5}$ & {$[20]$} \\
\hline
\end{tabular}

Table 4: Minimal dilatations of braids

By using Theorem 4.1, Gabai, Meyerhoff and Milley proved the following. 


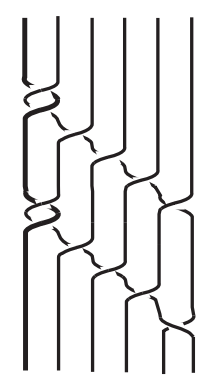

Figure 14: Braid $T_{6,2}$

Theorem 4.3 [11, Corollary 1.2] Let $M$ be a 1-cusped orientable hyperbolic 3manifold whose volume is at most 2.848. Then $M$ is one of $m 003, m 004, m 006$, $m 007, m 009, m 010, m 011, m 015, m 016$ and $m 017$. In particular, every 1 -cusped orientable hyperbolic 3-manifold whose volume is at most 2.848 can be obtained from the magic manifold by Dehn filling two cusps.

Among the 10 manifolds in Theorem 4.3, $m 006, m 007, m 015, m 017$ are nonfibered and the others are fibered; see Button [4] and Dunfield [7]. Each of the fibered manifolds in Theorem 4.3 has the second Betti number 1, and hence it admits a unique fibration. The entropies and normalized entropies of their fibrations are given in Table 5. Here are comments on the table.

(1) The first column "manifold" can be checked by using SnapPy [6].

(2) $m 003$ and $m 004$ are the figure 8 sister manifold and the figure 8 knot exterior respectively ([23, Table A.2] or [6]). It is well-known that each of $m 003$ and $m 004$ has the fiber $\Sigma_{1,1}$ and the monodromy of its fibration achieves the minimal dilatation $\delta_{1,1}=(3+\sqrt{5}) / 2$.

(3) SnapPy tells us that the once punctured torus bundles whose monodromies are given by $2 \times 2$ matrices

$$
\left(\begin{array}{ll}
3 & 2 \\
1 & 1
\end{array}\right), \quad\left(\begin{array}{cc}
-3 & -2 \\
1 & 1
\end{array}\right)
$$

are homeomorphic to $m 009$ and $m 010$ respectively. Hence their dilatations equal $2+\sqrt{3}$ which is the largest eigenvalue of:

$$
\left(\begin{array}{cc}
| \pm 3| & | \pm 2| \\
1 & 1
\end{array}\right)
$$


(4) The fibered class $a=(13,12,5) \in \operatorname{int}\left(C_{\Delta}\right)$ has boundary slopes $b_{\alpha}(a)=\frac{17}{-13}$, $b_{\beta}(a)=\frac{3}{-2}, b_{\gamma}(a)=\frac{5}{-1}$, and the genus of $F_{a}$ is 5 . As a consequence of this paper, we have that $N\left(\frac{3}{-2},-5\right)(\simeq m 011)$ has a fiber $\Sigma_{5,1}$ and the dilatation of the monodromy of its fibration equals $\lambda(a)$ which is the largest real root $\lambda_{(13,12,5)} \approx 1.1487$ of $f_{(13,12,5)}(t)$.

(5) The manifold $N\left(\frac{3}{-2}, \frac{8}{-3}\right)(\simeq m 016)$ is homeomorphic to the $(-2,3,7)$-pretzel knot exterior; see [23, Table A.4]. Because $a=(18,22,15) \in \operatorname{int}\left(C_{\Delta}\right)$ has the boundary slopes $b_{\alpha}(a)=\frac{37}{-18}, b_{\beta}(a)=\frac{3}{-2}, b_{\gamma}(a)=\frac{8}{-3}$ and the genus of $F_{a}$ equals 5 , the fiber of the fibration on $m 016$ is $\Sigma_{5,1}$. We see that the dilatation of the monodromy of its fibration equals $\lambda(a)$ which is the largest real root $\lambda_{(18,22,15)} \approx 1.1762$ of

$$
\begin{aligned}
& f_{(18,22,15)}(t) \\
& \quad=\left(t^{11}+1\right)\left(t^{4}-t^{3}+t^{2}-t+1\right)\left(t^{10}+t^{9}-t^{7}-t^{6}-t^{5}-t^{4}-t^{3}+t+1\right) .
\end{aligned}
$$

Namely it is the largest real root of the last factor. Thus the dilatation equals the so called Lehmer's number. The monodromy of the fibration is described in Leininger [22].

(6) The monodromy of the fibration on $m 016 \simeq N\left(\frac{3}{-2}, \frac{8}{-3}\right)\left(\right.$ resp. $\left.m 011 \simeq N\left(\frac{3}{-2},-5\right)\right)$ can be extended to the pseudo-Anosov homeomorphism on the closed surface of genus 5 with dilatation $\lambda_{(18,22,15)}$ (resp. $\left.\lambda_{(13,12,5)}\right)$. This pseudo-Anosov is a representative of $\widehat{\phi}_{(18,22,5)} \in \widehat{\mathcal{M}}$ (resp. $\left.\widehat{\phi}_{(13,12,5)} \in \widehat{\mathcal{M}}\right)$. On the other hand, Lanneau and Thiffeault proved that $\delta_{5}^{+}$equals the Lehmer's number [21]. The pseudo-Anosov representative of $\widehat{\phi}_{(18,22,5)}$ has orientable stable foliation (see Lemma 3.20) and it achieves $\delta_{5}^{+}$. The mapping class $\widehat{\phi}_{(13,12,5)}$ was the example in $[1 ; 19]$ used to prove $\delta_{5}<\delta_{5}^{+}$.

\begin{tabular}{|cccc|}
\hline manifold & fiber $\Sigma$ & entropy $\log \lambda\left(\phi_{[\Sigma]}\right)$ & $|\chi(\Sigma)| \log \lambda\left(\phi_{[\Sigma]}\right)$ \\
\hline$m 003 \simeq N(1,-4)$ & $\Sigma_{1,1}$ & $\log \left(\frac{3+\sqrt{5}}{2}\right)$ & $\log \left(\frac{3+\sqrt{5}}{2}\right) \approx 0.9624$ \\
$m 004 \simeq N(1,2)$ & $\Sigma_{1,1}$ & $\log \left(\frac{3+\sqrt{5}}{2}\right)$ & $\log \left(\frac{3+\sqrt{5}}{2}\right) \approx 0.9624$ \\
$m 009 \simeq N(1,3)$ & $\Sigma_{1,1}$ & $\log (2+\sqrt{3})$ & $\log (2+\sqrt{3}) \approx 1.3169$ \\
$m 010 \simeq N(1,-5)$ & $\Sigma_{1,1}$ & $\log (2+\sqrt{3})$ & $\log (2+\sqrt{3}) \approx 1.3169$ \\
$m 011 \simeq N\left(\frac{3}{-2},-5\right)$ & $\Sigma_{5,1}$ & $\log \lambda_{(13,12,5)} \approx \log (1.1487)$ & $9 \log \lambda_{(13,12,5)} \approx 1.2484$ \\
$m 016 \simeq N\left(\frac{3}{-2}, \frac{8}{-3}\right)$ & $\Sigma_{5,1}$ & $\log \lambda_{(18,22,15)} \approx \log (1.1762)$ & $9 \log \lambda_{(18,22,15)} \approx 1.4612$ \\
\hline
\end{tabular}

Table 5: Normalized entropies $|\chi(\Sigma)| \log \lambda\left(\phi_{[\Sigma]}\right)$ of 1-cusped hyperbolic fibered 3-manifolds with volume $\leq 2.848$ 
The number $\log (3+\sqrt{5} / 2)$ is the minimal normalized entropy among 1 -cusped hyperbolic fibered 3-manifolds with volume less than or equal to 2.848; see Table 5. In practice, pseudo-Anosov homeomorphisms with small dilatation occur as monodromies of fibrations on fibered 3-manifolds with small volume; see [17]. Thus it is natural to ask the following.

Question 4.4 Let $M$ be a 1-cusped hyperbolic fibered 3-manifold with the second Betti number 1. Is it true that the normalized entropy of the monodromy of the fibration on $M$ is greater than or equal to $\log ((3+\sqrt{5}) / 2)$ ? Is it true that the equality is achieved only by either $m 003$ or $m 004$ ?

\section{Remarks}

We find from the next lemma that the set $\mathcal{M}$ is very large.

Lemma 5.1 Let $p \in \mathbb{N}$ and $q \in \mathbb{Z}$ be coprime such that $\frac{p}{q} \in \mathcal{H} y p$.

(1) Suppose $p / q \in(-\infty,-2)$ such that $p+2 q \neq 1$ (resp. suppose $\frac{p}{q} \in(-\infty,-2)$ such that $|q| \neq 1)$. Let $a \in S_{\beta}\left(\frac{p}{q}\right)$ be a primitive fibered class of $N$ such that $\bar{a} \in \operatorname{int}\left(C_{\Omega}\right)$, where $\Omega$ is an $S$-face (resp. $A$-face) of $N\left(\frac{p}{q}\right)$. Then $\phi_{a} \in \mathcal{M}$ for any such $a \in S_{\beta}\left(\frac{p}{q}\right)$ but finitely many exceptions.

(2) Suppose that $p / q \in(-2,0)$. Let $a \in S_{\beta}\left(\frac{p}{q}\right)$ be a primitive fibered class of $N$. Then $\phi_{a} \in \mathcal{M}$ for any such $a \in S_{\beta}\left(\frac{p}{q}\right)$ but finitely many exceptions.

(3) Suppose that $p / q \in(0, \infty)$ such that $p / q \neq 1$. Let $\Omega$ be an $S$-face of $N\left(\frac{p}{q}\right)$ if $|q|=1$ and let $\Omega$ be any face of $N\left(\frac{p}{q}\right)$ if $|q| \neq 1$. Let $a_{n} \in S_{\beta}\left(\frac{p}{q}\right)$ be a primitive fibered class of $N$ such that $\bar{a}_{n} \in \operatorname{int}\left(C_{\Omega}\right)$ for each $n$. If $\bar{a}_{i} \neq \bar{a}_{j}$ for $i \neq j$ and $\bar{a}_{n}$ converges projectively to a point of $\operatorname{int}(\Omega)$ as $n$ goes to $\infty$, then $\phi_{a_{n}} \in \mathcal{M}$ for large $n$.

Proof (2) If $\frac{p}{q} \in(-2,0)$, a primitive fibered class $\widehat{a} \in S_{\beta}\left(\frac{p}{q}\right)$ is $\operatorname{in} \operatorname{int}\left(C_{\widehat{\Delta}}\right)$, where $\widehat{\Delta}$ is a fibered face $N$ which is either $\Delta, \Delta^{\prime}, \Delta_{2}$ or $\Delta_{2}^{\prime}$. We note $\sigma\left(\operatorname{int}\left(C_{\widehat{\Delta}}\right) \cap S_{\beta}\left(\frac{p}{q}\right)\right)=$ $\operatorname{int}\left(C_{\Delta}\right) \cap S_{\alpha}\left(\frac{p}{q}\right)$ or $\operatorname{int}\left(C_{\Delta}\right) \cap S_{\beta}\left(\frac{p}{q}\right)$. By Lemma 2.4 and Remark 2.13, we may assume that $\sigma\left(\operatorname{int}\left(C_{\widehat{\Delta}}\right) \cap S_{\beta}\left(\frac{p}{q}\right)\right)=\operatorname{int}\left(C_{\Delta}\right) \cap S_{\beta}\left(\frac{p}{q}\right)$, and it is enough to consider primitive fibered classes $a \in \operatorname{int}\left(C_{\Delta}\right) \cap S_{\beta}\left(\frac{p}{q}\right)$.

If $\phi_{a} \notin \mathcal{M}$, then one of the following two cases occur:

- $a \in S_{\alpha}\left(\frac{t}{u}\right) \cap S_{\beta}\left(\frac{p}{q}\right)$ for some $\frac{t}{u}$ such that $|u|=1$.

- $a \in S_{\beta}\left(\frac{p}{q}\right) \cap S_{\gamma}\left(\frac{v}{w}\right)$ for some $\frac{v}{w}$ such that $v+2 w=1$. 
It suffices to show that the following two sets are finite: fixing $\frac{p}{q}$,

$$
\begin{aligned}
& \left\{[x, y] \in \operatorname{int}(\Delta) \cap S_{\alpha}\left(\frac{t}{u}\right) \cap S_{\beta}\left(\frac{p}{q}\right)|| u \mid=1\right\}, \\
& \left\{[x, y] \in \operatorname{int}(\Delta) \cap S_{\beta}\left(\frac{p}{q}\right) \cap S_{\gamma}\left(\frac{v}{w}\right) \mid v+2 w=1\right\} .
\end{aligned}
$$

One can prove the first set is finite as follows. Recall from Lemma 2.12 that

$$
\begin{aligned}
& \Delta \cap S_{\alpha}(r)=\left\{[x, y] \in \Delta \mid y=\left(\frac{1+r}{-2}\right) x+\frac{1}{2}\right\}, \\
& \Delta \cap S_{\beta}(r)=\left\{[x, y] \in \Delta \mid y=\left(\frac{-2}{1+r}\right) x+\frac{1}{1+r}\right\} .
\end{aligned}
$$

Suppose that $|u|=1$. Then

$$
\left(\Delta \cap S_{\alpha}\left(\frac{t}{u}\right)\right) \cap\left(\Delta \cap S_{\beta}\left(\frac{p}{q}\right)\right)=\varnothing \quad \text { for large }\left|\frac{t}{u}\right| .
$$

The finiteness of the second set can be proved similarly.

The proof of (1) is similar to that of (2).

(3) Let us consider the case that $\Omega$ is an $S$-face. The primitive fibered classes $a_{n}$ enjoying the assumption of the claim must be in the interior of the cone over the fibered face $\Delta_{1}$ or $\Delta_{1}^{\prime}$. The images of $\operatorname{int}\left(C_{\Delta_{1}}\right) \cap S_{\beta}\left(\frac{p}{q}\right)$ and $\operatorname{int}\left(C_{\Delta_{1}^{\prime}}\right) \cap S_{\beta}\left(\frac{p}{q}\right)$ under $\sigma$ are the same, and it is the set $\operatorname{int}\left(C_{\Delta}\right) \cap S_{\gamma}\left(\frac{p}{q}\right)$. Hence it is enough to consider the primitive fibered classes $a_{n} \in \operatorname{int}\left(C_{\Delta}\right) \cap S_{\gamma}\left(\frac{p}{q}\right)$ which enjoy the assumption of the claim (3).

We consider the following two infinite sets: fixing $\frac{p}{q}$,

$$
\begin{aligned}
& \left\{[x, y] \in \operatorname{int}(\Delta) \cap S_{\alpha}\left(\frac{t}{u}\right) \cap S_{\gamma}\left(\frac{p}{q}\right)|| u \mid=1\right\}, \\
& \left\{[x, y] \in \operatorname{int}(\Delta) \cap S_{\beta}\left(\frac{t}{u}\right) \cap S_{\gamma}\left(\frac{p}{q}\right)|| u \mid=1\right\} .
\end{aligned}
$$

Consider the case $\frac{p}{q} \in(0,1)$. If $\frac{t}{u} \in(-\infty,-1]$, then

$$
\operatorname{int}(\Delta) \cap S_{\alpha}\left(\frac{t}{u}\right) \cap S_{\gamma}\left(\frac{p}{q}\right)=\varnothing \quad \text { and } \quad \operatorname{int}(\Delta) \cap S_{\beta}\left(\frac{t}{u}\right) \cap S_{\gamma}\left(\frac{p}{q}\right)=\varnothing
$$

(see Figure 15(left)). Suppose $\frac{t}{u} \in(-1, \infty)$ such that $|u|=1$. (Then $\frac{t}{u}=\frac{t}{1} \in[0, \infty)$.) Fixing $t_{0}>0$, then we have that the sets

$\left\{\operatorname{int}(\Delta) \cap S_{\alpha}\left(\frac{t}{1}\right) \cap S_{\gamma}\left(\frac{p}{q}\right) \mid t \in \mathbb{N}, t<t_{0}\right\}, \quad\left\{\operatorname{int}(\Delta) \cap S_{\beta}\left(\frac{t}{1}\right) \cap S_{\gamma}\left(\frac{p}{q}\right) \mid t \in \mathbb{N}, t<t_{0}\right\}$ are finite clearly. Observe that for large $t \in \mathbb{N}$, then we have

$$
\operatorname{int}(\Delta) \cap S_{\alpha}\left(\frac{t}{1}\right) \cap S_{\gamma}\left(\frac{p}{q}\right) \neq \varnothing \quad \text { and } \quad \operatorname{int}(\Delta) \cap S_{\beta}\left(\frac{t}{1}\right) \cap S_{\gamma}\left(\frac{p}{q}\right) \neq \varnothing,
$$


but each point of these nonempty sets goes to a point of $\partial \Delta \cap S_{\beta}\left(\frac{p}{q}\right)$ as $t$ goes to $\infty$ (see Figure 15(right)). This leads to the claim (3).

The proof in the case $\frac{p}{q} \in(1, \infty)$ is similar.

By a similar argument, one can prove the claim (3) when $\Omega$ is an $A$-face.
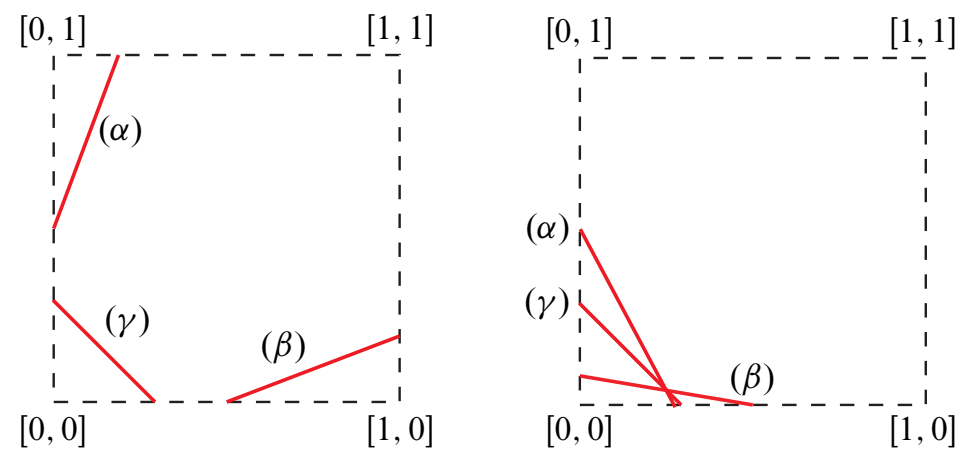

Figure 15: (Left) $t / u \in(-\infty,-1)$, (right) $t / u \in(-1, \infty)$

$\left[(\alpha):=\operatorname{int}(\Delta) \cap S_{\alpha}\left(\frac{t}{u}\right),(\beta):=\operatorname{int}(\Delta) \cap S_{\beta}\left(\frac{t}{u}\right),(\gamma):=\operatorname{int}(\Delta) \cap S_{\gamma}\left(\frac{p}{q}\right)\right]$

In contrast with Lemma 3.28, we have the following.

Lemma 5.2 Suppose that $p / q \neq 1$. For a primitive fibered class $a \in S_{\alpha}\left(\frac{p}{q}\right)$, the number of the boundary components of $F_{\bar{a}}$ for $\bar{a} \in H_{2}\left(N_{\alpha}\left(\frac{p}{q}\right), \partial N_{\alpha}\left(\frac{p}{q}\right)\right)$ is bounded by $2|p|+2|q|$.

Proof Suppose that $a=(x, y, z) \in S_{\alpha}\left(\frac{p}{q}\right)$ is a primitive fibered class. The number of the boundary components of $F_{\bar{a}}$ equals $\operatorname{gcd}(y, z+x)+\operatorname{gcd}(z, x+y)$.

We shall prove that $\operatorname{gcd}(y, z+x) \leq|p|+|q|$. The inequality $\operatorname{gcd}(z, x+y) \leq|p|+|q|$ can be proved by the same argument. Since $-p x=q(y+z)$, there exists an integer $k$ such that $x=-q k$ and $y+z=p k$. Hence $z=p k-y$.

Claim 5.3 $\operatorname{gcd}(y, k)=\operatorname{gcd}(z, k)=1$

Proof of Claim 5.3 Suppose that $\operatorname{gcd}(y, k)=\ell>1$. Then $y=y^{\prime} \ell$ and $k=k^{\prime} \ell$ for some integers $y^{\prime}$ and $k^{\prime}$. One sees that

$$
\operatorname{gcd}(z, k)=\operatorname{gcd}(p k-y, k)=\operatorname{gcd}\left(p k^{\prime} \ell-y^{\prime} \ell, k^{\prime} \ell\right) \geq \ell .
$$

Thus $\ell$ is a factor of $y, z$ and $k$. Recall $x=-q k$. This implies $\ell$ is also a factor of $x$. Thus $\operatorname{gcd}(x, y, z) \geq \ell$. Since $(x, y, z)$ is a primitive class, this is a contradiction. 
One has $\operatorname{gcd}(y, z+x)=\operatorname{gcd}(y,(p-q) k-y)=\operatorname{gcd}(y,(p-q) k)$. Since $\operatorname{gcd}(y, k)=1$, one obtains $\operatorname{gcd}(y,(p-q) k)=\operatorname{gcd}(y, p-q) \leq p-q \leq|p|+|q|$. This completes the proof Lemma 5.2.

The following is an application of Lemmas 5.1 and 5.2.

Proposition 5.4 For each $r \in \mathcal{H} y p \backslash\{1\}$, let $\Omega$ be a fibered face of $N(r)$ which enjoys (*) in Theorem 1.11. Let $a_{n} \in S_{\beta}(r)$ be a primitive fibered class such that $\bar{a}_{n} \in \operatorname{int}\left(C_{\Omega}\right)$ for each $n \in \mathbb{N}$. Suppose that $\bar{a}_{n}$ converges projectively to a unique point of $\operatorname{int}(\Omega)$ which achieves $\min \operatorname{Ent}(N(r), \Omega)$. Then $\phi_{a_{n}} \in \mathcal{M}$ for large $n$. Moreover

$$
\min \operatorname{Ent}(N(r), \Omega)=\lim _{n \rightarrow \infty} \operatorname{Ent}\left(\widehat{\phi}_{a_{n}}\right) .
$$

Proof The first assertion is immediate from Lemma 5.1. By the assumption of $\bar{a}_{n}$,

$$
\min \operatorname{Ent}(N(r), \Omega)=\lim _{n \rightarrow \infty} \operatorname{Ent}_{r}\left(\bar{a}_{n}\right)\left(=\lim _{n \rightarrow \infty} \operatorname{Ent}\left(\phi_{\bar{a}_{n}}\right)\right) .
$$

Since $\phi_{a_{n}} \in \mathcal{M}$ for large $n, \lambda_{r}\left(\bar{a}_{n}\right)\left(=\lambda\left(\phi_{\bar{a}_{n}}\right)\right)=\lambda\left(\widehat{\phi}_{a_{n}}\right)$ for large $n$. By Lemma 5.2, the number of the boundary components of the minimal representative of $\bar{a}_{n}$ is bounded. Thus the normalized entropy $\operatorname{Ent}\left(\widehat{\phi}_{a_{n}}\right)$ of $\widehat{\phi}_{a_{n}}$ tends to $\min \operatorname{Ent}(N(r), \Omega)$ as $n$ tends to $\infty$.

\section{References}

[1] J W Aaber, N Dunfield, Closed surface bundles of least volume, Algebr. Geom. Topol. 10 (2010) 2315-2342 MR2745673

[2] I Agol, The minimal volume orientable hyperbolic 2-cusped 3-manifolds, Proc. Amer. Math. Soc. 138 (2010) 3723-3732 MR2661571

[3] I Agol, Ideal triangulations of pseudo-Anosov mapping tori, from: "Topology and geometry in dimension three", (W Li, L Bartolini, J Johnson, F Luo, R Myers, J H Rubinstein, editors), Contemp. Math. 560, Amer. Math. Soc. (2011) 1-17 MR2866919

[4] J O Button, Fibred and virtually fibred hyperbolic 3-manifolds in the censuses, Experiment. Math. 14 (2005) 231-255 MR2169525

[5] J-H Cho, J-Y Ham, The minimal dilatation of a genus-two surface, Experiment. Math. 17 (2008) 257-267 MR2455699

[6] M Culler, N M Dunfield, J R Weeks, SnapPy, a computer program for studying the geometry and topology of 3-manifolds Available at http://snappy.computop.org

[7] NM Dunfield, Which cusped census manifolds fiber? Available at http:// www.math.uiuc.edu/ nmd/snappea/tables/mflds_which_fiber

[8] B Farb, C J Leininger, D Margalit, Small dilatation pseudo-Anosov homeomorphisms and 3-manifolds, Adv. Math. 228 (2011) 1466-1502 MR2824561 
[9] A Fathi, F Laudenbach, V Poenaru, Travaux de Thurston sur les surfaces, Astérisque 66, Société Mathématique de France, Paris (1979) MR568308

[10] D Fried, Flow equivalence, hyperbolic systems and a new zeta function for flows, Comment. Math. Helv. 57 (1982) 237-259 MR684116

[11] D Gabai, R Meyerhoff, P Milley, Minimum volume cusped hyperbolic three-manifolds, J. Amer. Math. Soc. 22 (2009) 1157-1215 MR2525782

[12] J-Y Ham, W T Song, The minimum dilatation of pseudo-Anosov 5-braids, Experiment. Math. 16 (2007) 167-179 MR2339273

[13] M Handel, The forcing partial order on the three times punctured disk, Ergodic Theory Dynam. Systems 17 (1997) 593-610 MR1452182

[14] E Hironaka, Small dilatation mapping classes coming from the simplest hyperbolic braid, Algebr. Geom. Topol. 10 (2010) 2041-2060 MR2728483

[15] E Hironaka, E Kin, A family of pseudo-Anosov braids with small dilatation, Algebr. Geom. Topol. 6 (2006) 699-738 MR2240913

[16] N V Ivanov, Coefficients of expansion of pseudo-Anosov homeomorphisms, Zap. Nauchn. Sem. Leningrad. Otdel. Mat. Inst. Steklov. (LOMI) 167 (1988) 111-116, 191 MR964259

[17] E Kin, S Kojima, M Takasawa, Entropy versus volume for pseudo-Anosovs, Experiment. Math. 18 (2009) 397-407 MR2583541

[18] E Kin, M Takasawa, Pseudo-Anosov braids with small entropy and the magic 3manifold, Comm. Anal. Geom. 19 (2011) 705-758 MR2880213

[19] E Kin, M Takasawa, Pseudo-Anosovs on closed surfaces having small entropy and the Whitehead sister link exterior, J. Math. Soc. Japan 65 (2013) 411-446 MR3055592

[20] E Lanneau, J-L Thiffeault, On the minimum dilatation of braids on punctured discs, Geom. Dedicata 152 (2011) 165-182 MR2795241

[21] E Lanneau, J-L Thiffeault, On the minimum dilatation of pseudo-Anosov homeromorphisms on surfaces of small genus, Ann. Inst. Fourier (Grenoble) 61 (2011) 105-144 MR2828128

[22] C J Leininger, On groups generated by two positive multi-twists: Teichmüller curves and Lehmer's number, Geom. Topol. 8 (2004) 1301-1359 MR2119298

[23] B Martelli, C Petronio, Dehn filling of the "magic" 3-manifold, Comm. Anal. Geom. 14 (2006) 969-1026 MR2287152

[24] S Matsumoto, Topological entropy and Thurston's norm of atoroidal surface bundles over the circle, J. Fac. Sci. Univ. Tokyo Sect. IA Math. 34 (1987) 763-778 MR927609

[25] T Matsuoka, Braids of periodic points and a 2-dimensional analogue of Sharkovskiu’s ordering, from: "Dynamical systems and nonlinear oscillations", (G Ikegami, editor), World Sci. Adv. Ser. Dynam. Systems 1, World Sci. Publishing, Singapore (1986) 58-72 MR854304

[26] C T McMullen, Polynomial invariants for fibered 3-manifolds and Teichmüller geodesics for foliations, Ann. Sci. École Norm. Sup. 33 (2000) 519-560 MR1832823 
[27] H Minakawa, Examples of pseudo-Anosov homeomorphisms with small dilatations, J. Math. Sci. Univ. Tokyo 13 (2006) 95-111 MR2277516

[28] R C Penner, Bounds on least dilatations, Proc. Amer. Math. Soc. 113 (1991) 443-450 MR1068128

[29] W T Song, K H Ko, J E Los, Entropies of braids, J. Knot Theory Ramifications 11 (2002) 647-666 MR1915500

[30] W P Thurston, Hyperbolic structures on 3-manifolds II: Surface groups and 3manifolds which fiber over the circle arXiv:math/9801045

[31] W P Thurston, A norm for the homology of 3-manifolds, Mem. Amer. Math. Soc. 59 (1986) 99-130 MR823443

[32] C-Y Tsai, The asymptotic behavior of least pseudo-Anosov dilatations, Geom. Topol. 13 (2009) 2253-2278 MR2507119

[33] C-Y Tsai, Minimal pseudo-Anosov translation lengths on the Teichmuller space, PhD thesis, University of Illinois (2010) MR2941648 Available at http:// search.proquest.com//docview/776906819

[34] R W Venzke, Braid forcing, hyperbolic geometry, and pseudo-Anosov sequences of low entropy, PhD thesis, California Institute of Technology (2008) MR3078552 Available at http://search.proquest.com//docview/1080955604

[35] A Y Zhirov, On the minimum dilation of pseudo-Anosov diffeomorphisms of a double torus, Uspekhi Mat. Nauk 50 (1995) 197-198 MR1331364

EK: Department of Mathematics, Graduate School of Science, Osaka University Toyonaka, Osaka 560-0043, Japan

SK, MT: Department of Math. and Computing Sciences, Tokyo Institute of Technology Ohokayama, Meguro, Tokyo 152-8552, Japan

kin@math.sci.osaka-u.ac.jp, sadayosi@is.titech.ac.jp,

takasawa@is.titech.ac.jp

http://www.math.sci.osaka-u.ac.jp/ kin/, http://www.is.titech.ac.jp/ sadayosi/, http://www.is.titech.ac.jp/ takasawa/

Received: 18 October $2011 \quad$ Revised: 15 February 2013 